\title{
Numerical Simulation of Pulse-Tube Refrigerators
}

Irina Lyulina 
Copyright (C2004 by Irina Lyulina, Eindhoven, The Netherlands.

All rights are reserved. No part of this publication may be reproduced, stored in a retrieval system, or transmitted, in any form or by any means, electronic, mechanical, photocopying, recording or otherwise, without prior permission of the author.

Printed by Eindhoven University Press

Cover design: JWL Producties

CIP-DATA LIBRARY TECHNISCHE UNIVERSITEIT EINDHOVEN

Lyulina, Irina

Numerical Simulation of Pulse-Tube Refrigerators

by Irina Lyulina. -

Eindhoven : Technische Universiteit Eindhoven, 2004. Proefschrift. ISBN 90-386-0982-5

NUR 919

Subject headings: nonlinear partial differential equations, numerical simulation, computational fluid dynamics, compressible flow, cryogenics 2000 Mathematics Subject Classification: 65M06,81T80,76MXX 


\section{Numerical Simulation of Pulse-Tube Refrigerators}

\section{PROEFSCHRIFT}

ter verkrijging van de graad van doctor aan de Technische Universiteit Eindhoven, op gezag van de Rector Magnificus, prof.dr. R.A. van Santen, voor een commissie aangewezen door het College voor Promoties in het openbaar te verdedigen op woensdag 19 januari 2005 om 16.00 uur

door

\section{Irina Lyulina}

geboren te St. Petersburg, Rusland 
Dit proefschrift is goedgekeurd door de promotoren:

prof.dr. R.M.M. Mattheij

en

prof.dr. A.T.A.M. de Waele

\section{Copromotor:}

dr.ir. A.S. Tijsseling 


\section{Contents}

1 Introduction 1

1.1 Pulse-tube refrigerators: history and applications . . . . . . . . 1

1.2 Different modelling approaches . . . . . . . . . . . . . . 3

1.3 Our objectives . . . . . . . . . . . . . . . . . . . . . . 5

1.4 Outline of the thesis . . . . . . . . . . . . . . . . . . . 6

2 Modelling 9

2.1 Physical model. . . . . . . . . . . . . . . . . . . . . 9

2.2 Mathematical model. . . . . . . . . . . . . . . . . . . 12

2.3 One-dimensional formulation . . . . . . . . . . . . . . . 13

2.3.1 Governing equations . . . . . . . . . . . . . . . . 13

2.3.2 Low-Mach-number approximation . . . . . . . . . . . 18

2.3.3 Boundary and initial conditions . . . . . . . . . . . . 20

2.3.4 Two-dimensional corrections to the one-dimensional model . . 23

2.4 Two-dimensional formulation . . . . . . . . . . . . . . . 24

2.4.1 Governing equations . . . . . . . . . . . . . . . . 24

2.4.2 Low-Mach-number approximation . . . . . . . . . . . 26

2.4 .3 Wall model. . . . . . . . . . . . . . . . . . . . 29

2.4.4 Boundary and initial conditions . . . . . . . . . . . . 30

3 Numerical solution methods for 1D equations 33

3.1 Velocity and temperature computation . . . . . . . . . . . . 33

3.2 Pressure correction algorithms for the $1 \mathrm{D}$ case . . . . . . . . . . 37

3.2.1 A model problem . . . . . . . . . . . . . . . . . . 38

3.2.2 Pulse tube flow . . . . . . . . . . . . . . . . . . . . . . . 49

3.3 Local grid refinement . . . . . . . . . . . . . . . . . . 51

3.3.1 A model problem . . . . . . . . . . . . . . . . . . . . 52

3.3.2 Two-grid LUGR with fixed refinement area . . . . . . . . 53

3.3.3 Two-grid LUGR with moving refinement area . . . . . . . 55

4 Numerical solution methods for 2D equations $\quad 57$ 
4.1 Temperature computation for the $2 \mathrm{D}$ case . . . . . . . . . . . . 57

4.2 Pressure correction algorithm for the 2D case. . . . . . . . . . . . 63

4.3 Numerical examples. . . . . . . . . . . . . . . . . . . . . . . . . . . . 67

4.3.1 Hagen-Poiseuille flow in a circular pipe . . . . . . . . . 67

4.3.2 Starting flow in a circular pipe. . . . . . . . . . . . . 70

4.3.3 Flow due to an oscillating pressure gradient. . . . . . . . 70

4.3.4 Flow over a backward-facing step . . . . . . . . . . . . 74

4.3.5 Temperature distribution in fully developed pipe flow . . . . 76

5 Flow and heat transfer computations for the pulse tube $\mathbf{8 1}$

5.1 One-dimensional results . . . . . . . . . . . . . . . . . 81

5.1 .1 Velocity . . . . . . . . . . . . . . . . . . . . . 82

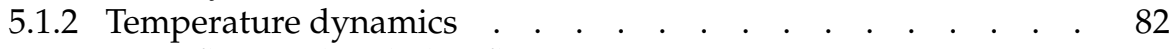

5.1.3 Mass flow and enthalpy flow . . . . . . . . . . . . 88

5.2 Two-dimensional results . . . . . . . . . . . . . . . . . . . . . . 92

5.2.1 Temperature and flow computations . . . . . . . . . . 92

5.2.2 Fluid-wall interaction . . . . . . . . . . . . . . . . 96

6 Concluding remarks and future work 103

Appendix A. Parameters for a typical single-inlet pulse-tube refrigerator 105

$\begin{array}{lr}\text { Bibliography } & 107\end{array}$

$\begin{array}{ll}\text { Nomenclature } & 115\end{array}$

$\begin{array}{lr}\text { Index } & 119\end{array}$

$\begin{array}{lr}\text { Summary } & 121\end{array}$

$\begin{array}{ll}\text { Samenvatting } & 123\end{array}$

$\begin{array}{ll}\text { Acknowledgements } & 125\end{array}$

$\begin{array}{lr}\text { Curriculum Vitae } & 127\end{array}$ 


\section{Introduction}

\subsection{Pulse-tube refrigerators: history and applications}

The pulse tube is a relatively new type of refrigerator. It was introduced in 1964 by Gifford and Longworth [20]. Their machine worked by the cyclic compression and expansion of helium gas in a half-open tube. Due to the heat exchange between gas, tube walls and two heat exchangers a temperature difference arose over the tube. In their first report a cold end temperature of $150 \mathrm{~K}$ was achieved. At that time the pulse tube was abandoned as a useful cooler because of its inefficiency. The performance of these devices, today known as basic pulse tubes, was limited, typically reaching temperatures of about $120 \mathrm{~K}$.

A significant improvement was made in 1984 by Mikulin et al. [51], who introduced the orifice pulse tube. They modified the half-open tube by connecting an orifice and a reservoir to the hot end. Due to this modification the performance of the pulse tube increased and for the first time it became comparable to the performance of practical coolers (Stirling cycle, Gifford-McMahon and Joule-Thomson cryocoolers). In 1986 Radebaugh et al. [56] reached $60 \mathrm{~K}$ with an orifice pulse tube. Since then the improvement in efficiency and in performance went fast. In 1990 Zhu et al. [95] added a bypass with second orifice to the device and introduced the double-inlet pulse-tube refrigerator. In 1994 Matsubara [48] used this technique to reach temperatures below $4 \mathrm{~K}$. By the end of the 1990s, temperatures below $2 \mathrm{~K}$ with a three-stage pulse tube and with ${ }^{3} \mathrm{He}$ as working fluid had been reached [90].

Two major classes of pulse-tube refrigerators are currently under development. The first class, known as "low frequency" or "G-M style" pulse tube, is a variant of the Gifford-McMahon cryocooler. G-M style pulse-tube coolers operate at low frequencies, typically less than $5 \mathrm{~Hz}$, and at high pressure ratios up to 5:1. They use a conventional oil-flooded G-M compressor and a valve located near the cold end to convert the flow of helium from the compressor to a low frequency pressure variation.

The second class of pulse-tube refrigerators is known as "high frequency" or "Stir- 


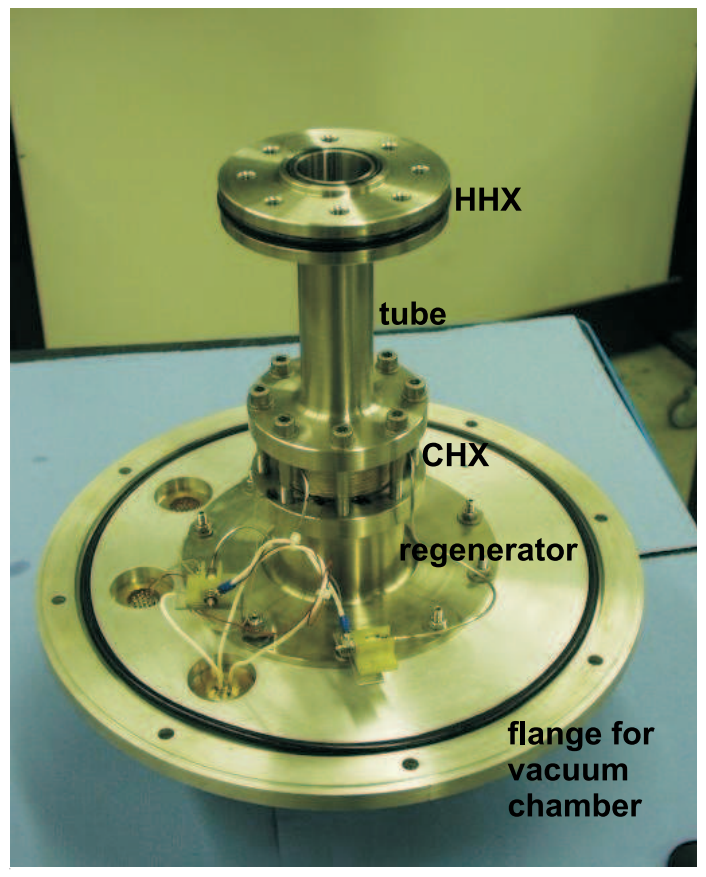

Figure 1.1: Pulse-tube refrigerator (Stirling Cryogenics \& Refrigeration B.V.) Main parts of the system are a regenerator, a cold heat exchanger (CHX), a tube and a hot heat exchanger (HHX).

ling type" pulse tube. These cryocoolers operate at much higher frequencies (20 to 80 $\mathrm{Hz}$ ) and much lower pressure ratios 1.4:1, than G-M style pulse tubes. Stirling-type pulse-tube refrigerators use a valveless compressor to generate the oscillating pressure for the cooling cycle. The compressor is usually driven by a linear motor. With this type of motor, it is possible to make a cooler with a continuous duty lifetime in excess of ten years.

At present pulse-tube refrigerators are competitive to Stirling and Gifford-McMahon coolers, both in terms of temperature range and efficiency. Pulse-tube refrigerators have no moving parts in the low temperature region, resulting in lower mechanical vibration and longer life compared to other coolers. From the early eighties, NASA's Ames Research Center initiated the research on pulse tubes, recognizing its potential for space applications. Pulse-tube refrigerators are widely used in the aerospace industry, for cooling sensitive detectors in satellites and in Earth observation instruments.

At the end of 20th century, the new technologies were transferred to non-aerospace applications, in particular to the electronic and computer industries. Pulse-tube refrigerators are used for cooling high-temperature superconductors in mobile communication, sensors in infrared cameras in military applications and high-power chips 
in computers. Medicine is another application area for pulse-tube refrigerators. Pulse tubes are used to cool superconducting magnets in diagnostic imaging systems (MRI), replacing the liquid-helium bath. This improves the quality of images due to the reduction of vibration and it makes MRI systems smaller and cheaper.

The development of pulse-tube cryocoolers is still at an early stage. Only few models are currently in production. A prototype, built at Stirling Cryogenics \& Refrigeration B.V., is shown in Figure 1.1. However, pulse-tube cryocoolers are beginning to replace the older types of cryocoolers in a wide variety of applications. Advantages such as simplicity, low cost and reliability, combined with high performance, have resulted in an extensive study of pulse tubes in recent years.

\subsection{Different modelling approaches}

To predict the performance of pulse-tube refrigerators different analytical and numerical models have been developed. Some models treat the entire system, some concentrate on specific parts of the system where significant energy losses are to be expected.

Thermodynamical models [66], [77], [78] use the laws of thermodynamics to analyse the performance of a pulse tube. Such models are important for understanding the physical processes occurring in the pulse tube. They explain why pulse-tube refrigerators cool and they give a good qualitative prediction of the performance. These thermodynamical models are time-averaged. For a more accurate prediction of pulse tube performance one has to analyse compressible oscillating gas flow using full timedependent models of fluid dynamics.

The system of conservation laws forms the basis of fluid dynamical models. In [65] a one-dimensional system of conservation laws was studied using analytical techniques. Regular asymptotic expansions were employed to derive simplified equations and approximate solutions. The expressions obtained gave insight into the underlying fluid mechanics and heat transfer.

Several attempts to linearise the conservation laws have been made. One of the possible approaches is harmonic analysis, see [29], [76]. Harmonic time dependence is used and all variables of the system are expanded in harmonic series. Then the onedimensional conservation equations are solved through an expansion series solution. Harmonic analysis is widely used as a pulse tube development tool. One of the main benefits of this approach is its capability to perform rapid optimisations in respect to dimensions and operational conditions of the pulse-tube cooler. However, this approach is restricted to small-amplitude harmonic pressure variations in the system.

In [3], [4], [52] a two-dimensional model for the tapered tube section of a pulsetube refrigerator has been proposed. Linearised conservation equations were solved analytically. Mean temperature profiles for gas and tube wall were obtained. The effects of operating frequency, taper angle, displacement volume ratio and phase angle between velocities at the ends of the tube on the net energy flow were studied.

In the series of articles [42], [43], [44] an anelastic approximation of the one- and two-dimensional conservation equations for the tube was used. An anelastic approx- 
imation means "filtering of sound from the fluid equations". Its effect is to remove acoustic phenomena from the consideration. The anelastic approximation applies when the acoustic energy is small compared to the energy needed to compress and expand the gas, which generally is the case in pulse-tube refrigerators. Mathematically, it is an approximation of the fluid equations where pressure gradients are ignored in the mass conservation equation, but are retained in the momentum equation. It allows to construct a consistent set of linear differential equations amenable to a series expansion solution in the small parameter $\varepsilon$ - the ratio between gas displacement length and tube length. The leading-order problem and the mean-steady higher order equations were presented. The linearised equations were then simplified using complex embedding to eliminate the time dependence. The solutions for some limiting cases were discussed.

Due to the complexity of the conservation equations analytical solutions are essentially impossible. This is why numerical models are of great importance. In [84] a one-dimensional numerical model was used to describe an orifice pulse-tube refrigerator. All components of the system were considered with the basic assumptions: a) ideal gas, b) ideal heat exchangers, c) negligible axial heat conduction. The conservation equations were solved using the finite volume method. In later works [81], [82] real gas properties were taken into account. In [35] basically the same model was used for studying a double-inlet pulse tube and good agreement with experimental data was reported. In [36] the mixed Eulerian-Lagrangian method was applied for simulating and visualising one-dimensional gas flow in a two-stage pulse-tube cooler operating in the $4 \mathrm{~K}$ temperature region. The authors used a moving grid to follow the exact tracks of gas particles as they move with pressure oscillation in the pulse tube (Lagrangian approach). For the regenerator a fixed computational grid was used (Eulerian approach). The disadvantage of all one-dimensional models is its inability to accurately describe the effect of the fluid-wall interaction.

Several attempts have been made recently, see [30], [88], to solve the full set of conservation equations using commercially available computational fluid dynamics (CFD) software for compressible flows. In [30] gas flow inside the tube was treated as a two-dimensional axisymmetric flow. Cylindrical coordinates were used for the governing equations. The system of conservation laws was solved using the finite volume method with implicit time integration. The analytical solution valid for isothermal laminar flow was used in setting up the oscillating velocity radial profile. The simulation results suggest that the flow strongly depends on the applied inlet velocity profiles. Moreover, the internal velocity distribution induced by the inlet flow profile might be an important factor in determining the optimum compressor driving frequency. In [88] results of three-dimensional computations for gas flow in the tube are presented. Numerical simulation was used to determine the effect of threedimensional phenomena such as mass streaming and turbulence. The conclusion was that three-dimensional modelling tends to be very time consuming and therefore not applicable for real system optimisation. Neither work included dimensional analysis and identification of dimensionless numbers characterising the flow and heat transfer. However, use of CFD codes without mathematical insight easily leads to results of poor quality or even to erroneous results. In the case of the pulse tube, where we 
are dealing with low-Mach-number flow $\left(\mathrm{Ma} \sim 10^{-3}\right)$, the standard numerical methods for compressible flows are very inefficient and often do not function properly.

\subsection{Our objectives}

The current project is a result of collaboration between the Applied Physics Department (TU/e), Stirling Cryogenics \& Refrigeration B.V. and the Department of Mathematics and Computer Science (TU/e). For about ten years pulse-tube refrigerators have been the main subject of research in the Low Temperature Physics Group at the Department of Applied Physics (TU/e). The group has unique expertise in the field and a substantial number of publications [73-80], [87], [90]. The previous $\mathrm{PhD}$ projects [28], [66], [69] were focused on the theoretical and the experimental aspects of pulse-tube refrigerators.

Stirling Cryogenics \& Refrigeration B.V. is one of the main players in the global market for cooling equipment. The company is the world's biggest supplier of mediumand large size cooling installations based on the Stirling principle. The company's success is based on significant investment in innovative research and the pulse-tube refrigerator is considered as a promising cooling device in high-tech industrial applications.

The main objectives of the project are

- development of a mathematical model for simulating the heat transport in compressible oscillating gas flow in the tube section of a pulse-tube refrigerator,

- development of suitable numerical methods,

- implementation of the developed model in a simulation tool for calculating the dynamic characteristics of the cooling system.

The purpose of the modelling is to predict energy losses. Losses in the regenerator have been studied in [76-78]. Unfortunately, the losses in the tube are not as well known. A particular prominent shortcoming is the inability of models to predict the temperature profile in the tube, which is extremely difficult to measure. As was discussed in the previous section, the existing models and solution methods are not completely satisfactory. The limited progress is due to the complexity of the coupled differential equations representing mass, momentum and energy conservation. The problem under consideration has been the subject of research mostly in engineering and physical communities and there is need for a much more detailed mathematical study. Dimensional and multi-scale analyses are very effective techniques. These allow the derivation of a leading-order system, which is much simpler than the original system of conservation equations. The development of numerical methods, especially designed for a given flow problem, is a challenging task. The numerical model gives the possibility to consider not only limiting cases, but also more general problems. It provides a powerful tool for estimating the parameters of cooling systems, such as temperature, velocity, mass flow, enthalpy flow, which leads to a deeper 
understanding of heat transfer and fluid-wall interaction in oscillating flow. The simulation program is intended to give engineers a practical tool to estimate and reduce energy losses.

\subsection{Outline of the thesis}

This thesis contains six chapters. Chapter 2 gives a mathematical description of the processes occurring in a pulse tube. First the basic working principles of pulse-tube refrigerators are explained. Then a mathematical model for unsteady compressible viscous flow is formulated. It is based on the conservation laws written in differential form and using primitive variables. The dimensionless numbers that govern the given flow problem are identified. According to dimensional analysis, the tube conveys a low-Mach-number compressible flow. Asymptotic expansions in terms of powers of $\mathrm{M}^{2}$, a parameter related to the Mach number, are used in the derivation of leading-order equations. The asymptotic consideration reveals several key features of low-Mach-number compressible flows. Two physically distinct roles of pressure are distinguished: one as thermodynamic variable and one as hydrodynamic variable. The thermodynamic pressure appears in the energy equation and in the equation of state. It is spatially uniform and thus a function of time only. It is responsible for the global compression and expansion. The hydrodynamic pressure appears in the momentum equations and is induced by inertia and viscous forces. One-dimensional and two-dimensional cylindrical axisymmetric cases are considered. For both cases the resulting systems of equations, together with boundary and initial conditions, suitable for numerical solution, are given.

Chapter 3 presents the numerical methods for the solution of the resulting system of equations in the one-dimensional case. The governing equations are discretised using finite difference techniques. The computation of the velocity field is decoupled from the temperature computation by using values from the previous time level. The temperature equation is a convection-dominated equation. It is solved using stateof-the-art flux-limiter schemes in an attempt to preserve the steep temperature gradients that occur in the tube. The velocity in the one-dimensional case can be found from the so-called constraint equation. Although there is no need to compute the first-order hydrodynamic pressure in the one-dimensional case, we have done so to investigate different pressure-correction algorithms and problems related to pressurevelocity coupling. The optimal scheme found for the one-dimensional problem has been used in solving the two-dimensional system of equations. Our pressure correction scheme is based on the constraint equation and not on the continuity equation, which is different from the common approach in the simulation of compressible flow problems. The method has close resemblance with pressure correction in incompressible flow computations, except for a non-zero velocity divergence constraint. Finally, we explain how more accurate solutions can be obtained through grid refinement. Refining a grid throughout the entire computational domain can be expensive, particularly in multi-dimensions. Instead of applying a non-uniform locally refined grid we use several uniform grids with different mesh sizes that cover different parts of 
the domain. Besides the global grid, fine local grids are used which are also uniform. They cover only those parts of the domain that contain the high activity regions. The mesh size of each of these grids follows the activity of the solution. The solution is calculated on the composite grid which is the union of the uniform subgrids. This refinement strategy is known as local uniform grid refinement (LUGR).

Chapter 4 describes the numerical methods for the resulting system of equations in the two-dimensional case. The same techniques as in the one-dimensional case are used. The convection-diffusion equation for the gas temperature in the twodimensional model is combined with a heat conduction equation for the wall temperature. To study gas-wall interaction in detail, we employ a non-uniform boundarylayer type of grid. The flux-limiter scheme is to be modified for non-uniform grids. The pressure correction method, developed in the Chapter 3 and specially designed for low-Mach-number compressible flows, is extended to the two-dimensional case. The correctness of the numerical techniques described above is demonstrated with several examples: Hagen-Poiseuille flow, starting flow, flow due to an oscillating pressure gradient, backward facing step flow and Graetz heat transfer problems. The first three examples are well known problems with analytical solutions. The backward facing step flow is a benchmark problem widely used in computational fluid dynamics, much experimental and numerical information about this type of flow is available. The Graetz problems allow us to check our temperature computations.

Chapter 5 presents the computations for a typical pulse-tube refrigerator. First, the one-dimensional results are discussed. The velocity, temperature, mass flow rate and enthalpy flow are investigated for two driving pressures: sinusoidal and trapezoidal. The developed model is validated by comparing the results for sinusoidal pressure with a first-order harmonic analysis. The one-dimensional results of this chapter, presented in [46], [47], are obtained at low computational cost and they serve as a reference for the two-dimensional results. With the two-dimensional model the heat transfer between gas and wall is studied in detail. Thermal and viscous effects in radial direction are now taken into account.

Finally, Chapter 6 summarises the results of this study and suggests directions for future work. 


\section{Modelling}

In this chapter the mathematical description of the processes occurring in a pulse tube is given. First the basic working principles of pulse-tube refrigerators are explained. Then the mathematical model for unsteady compressible viscous flow is formulated. It is based on the conservation laws written in differential form and using primitive variables. The dimensionless numbers that govern the given flow problem are identified. According to dimensional analysis, the tube conveys a low-Mach-number compressible flow. Asymptotic expansions in terms of powers of $\mathrm{M}^{2}$, a dimensionless number related to the Mach number, are used in the derivation of leading-order equations. One-dimensional and two-dimensional cylindrical axisymmetrical cases are considered. For both cases the resulting systems of equations together with boundary and initial conditions, suitable for numerical solution, are given.

\subsection{Physical model}

The pulse-tube refrigerator works by the cyclic compression and expansion of a fixed quantity of gas, usually helium. The essential elements of a pulse-tube refrigerator are shown in Figure 2.1. Due to heat exchange between gas, regenerator, tube walls and the three heat exchangers, a temperature difference develops along the tube. The pressure oscillations in the system are generated by a piston or, alternatively, by switching valves. The aftercooler (AC), see Figure 2.1a, removes the heat of compression so that the regenerator can work more efficiently. The regenerator acts as follows: it absorbs heat from the gas in the compression part of the pressure cycle and it returns heat to the gas in the expansion part. To achieve this, the regenerator is filled with a matrix - some kind of solid porous material with a large heat capacity and a large heat-exchanging surface. The cold heat exchanger (CHX) is the coldest point of the system. Here the heat is extracted from the load to be cooled. In the tube, the compressible gas oscillates. If there is a suitable phase relationship between the pressure and the gas flow, heat will be transported from the cold end to the warm end. The hot heat exchanger (HHX) removes the heat carried through the tube. The 
a)

b)
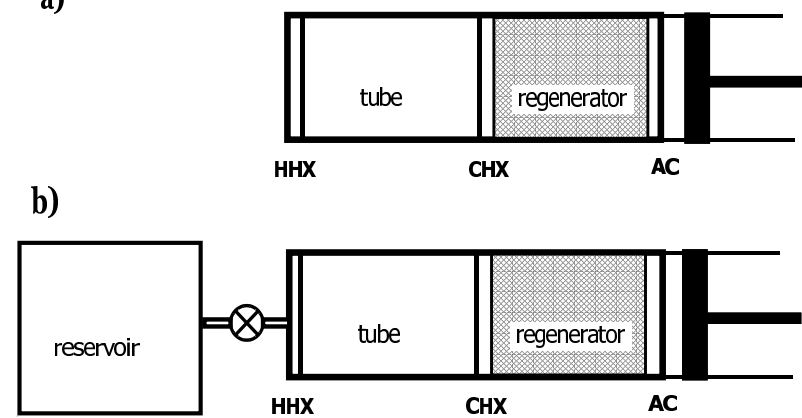

Figure 2.1: A schematic representation of Stirling-type pulse-tube refrigerators: (a) basic pulse tube and (b) orifice pulse tube. From right to left the system consists of a piston, an aftercooler (AC), a regenerator, a cold heat exchanger (CHX), a tube, a hot heat exchanger (HHX). In b) an orifice and a reservoir are added.

hot heat exchanger is maintained at ambient temperature. In the orifice design, see Figure 2.1b, the basic pulse tube has been modified by adding a reservoir (buffer) and a flow resistance (orifice). The orifice is a resistance valve that is tuned for optimal performance. The reservoir is large compared to the pulse tube. Gas flows through the orifice due to a pressure difference. More gas is contributing to the cooling power now and this improves the efficiency of the cooler. The cycle results in net enthalpy flow from the cold end to the hot end thus providing a continuous refrigeration effect.

An illustrative way of explaining the cooling process at the cold end is presented in Figure 2.2. The pressure in the tube is varied in four steps, see Figure 2.2a.

1. From a via $\mathbf{b}$ to $\mathbf{c}$. The compression step. The piston moves to the left. The orifice is closed. The pressure rises.

2. From $\mathbf{c}$ to $\mathbf{d}$. The orifice is open so that gas flows from the tube to the buffer. At the same time the piston moves to the left such that the pressure in the tube remains constant.

3. From $\mathbf{d}$ to $\mathbf{e}$. The expansion step. The piston moves to the right. The orifice is closed. The pressure drops.

4. From $\mathbf{e}$ via $\mathbf{f}$ to $\mathbf{a}$. The orifice is open so that gas flows from the buffer into the tube. At the same time the piston moves to the right such that the pressure in the tube remains constant.

We will follow a gas parcel, which is inside the regenerator at the start of the cycle (point a), see Figure 2.2b.

1. From $\mathbf{a}$ via $\mathbf{b}$ to $\mathbf{c}$. When the pressure rises, the gas parcel moves through the regenerator in the direction of $\mathrm{CHX}$. Its temperature remains constant due to 


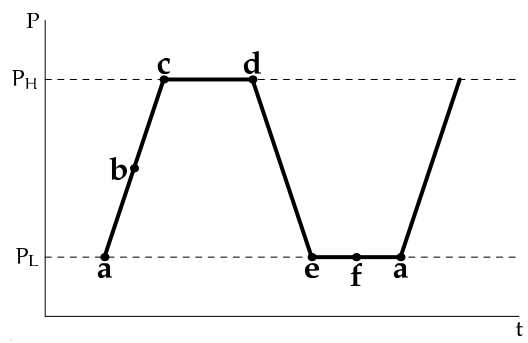

(a) pressure

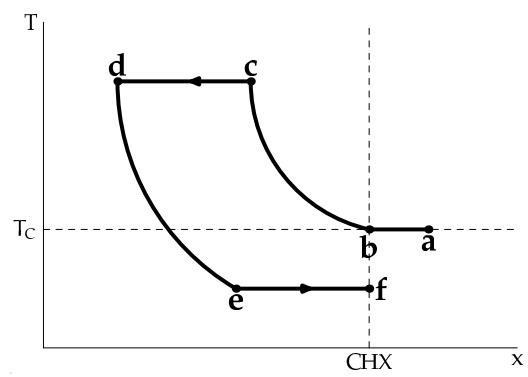

(b) temperature

Figure 2.2: Pressure in a pulse tube as a function of time and temperature of the gas element as a function of position.

good heat exchange with the regenerator material. At point $\mathbf{b}$ the gas parcel leaves the $\mathrm{CHX}$ and enters the tube with the temperature $\mathrm{T}_{\mathrm{C}}$. From $\mathbf{b}$ to $\mathbf{c}$ the gas parcel is compressed adiabatically, while it moves towards the orifice. Its temperature rises together with the pressure.

2. From $\mathbf{c}$ to $\mathbf{d}$. The gas parcel moves to the left. The pressure and the temperature are constant.

3. From $\mathbf{d}$ to $\mathbf{e}$. When the pressure drops the gas parcel moves back to the direction of CHX. As it is thermally isolated, its temperature drops together with the pressure. The temperature of the gas parcel at point $\mathbf{e}$ is below $\mathrm{T}_{\mathrm{C}}$.

4. From e via $\mathbf{f}$ to a. The gas parcel moves to the right. The pressure and the temperature are constant as long as the gas parcel is in the tube. At point $\mathbf{f}$ the gas parcel leaves the tube and enters the $\mathrm{CHX}$ with $\mathrm{T}<\mathrm{T}_{\mathrm{C}}$. When passing the $\mathrm{CHX}$ the gas extracts heat from it and warms up to the temperature $\mathrm{T}_{\mathrm{C}}$. That is when the cooling takes place. The amount of heat which the gas takes away from the heat exchanger is the cooling power. In the end of the cycle the gas parcel moves inside the regenerator to its original position. 


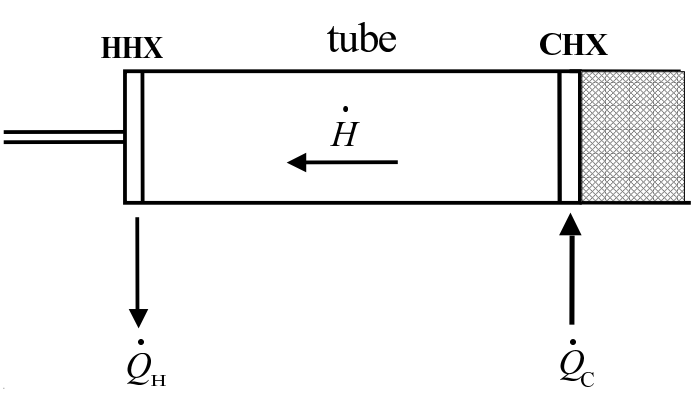

Figure 2.3: Net heat and enthalpy flows in an ideal tube.

Figure 2.3 shows the enthalpy flow in the pulse tube. At the hot heat exchanger heat $\dot{Q}_{H}$ is extracted from the system. At the cold heat exchanger heat $\dot{Q}_{C}$ is supplied to the system. There is a net enthalpy flow $\dot{H}$ from the cold heat exchanger to the hot heat exchanger. According to the first law of thermodynamics, the average cooling power is equal to the enthalpy flow in the pulse tube. To study the energy transfer from the cold end to the hot end, we will concentrate solely on the tube section of the pulse-tube refrigerator.

The flow in the pulse tube is an internal oscillating compressible flow. To avoid ambiguity, it must be mentioned that two different types of periodic flow are often referred to as oscillatory: pulsating flow and reciprocating flow. Pulsating flow is periodic but the flow direction never reverses. This happens when non-return valves prevent back flow (e.g. blood flow in arteries) or when small oscillations occur on top of a large steady flow. Reciprocating flow is the more difficult case because the flow direction reverses periodically. In pulse-tube refrigerators we are dealing with a reciprocating type of oscillating flow.

\subsection{Mathematical model}

The mathematical modelling of a physical system begins with identifying the desired level of description. A set of basic variables is introduced that completely defines the system at the chosen level. The basic physical laws are then formulated in terms of the chosen variables.

For the analysis of fluid flows at a macroscopic level molecular structures and molecular motions are ignored and the fluid is regarded as a continuum. The fluid behaviour is described in terms of macroscopic properties, such as velocity, pressure, density, temperature and their space and time derivatives. The governing equations are mathematical statements of the conservation laws of physics:

- Continuity: the mass is conserved.

- Newton's law: the rate of change of momentum equals the sum of forces. 
- First law of thermodynamics: the rate of change of energy equals the difference of the rate of heat addition and the rate of work done.

The conservation equations of fluid dynamics can be formulated in many equivalent ways, see [8]. For general compressible fluid flow, differential forms of these equations in terms of so-called "primitive" variables, namely density $\rho(x, t)$, velocity $\mathbf{u}(\mathbf{x}, \mathbf{t})$, temperature $T(\mathbf{x}, \mathbf{t})$ and pressure $\mathbf{p}(\mathbf{x}, \mathbf{t})$, are

conservation of mass (continuity equation):

$$
\frac{\mathrm{D} \rho}{\mathrm{D} t}+\rho \nabla \cdot \mathbf{u}=0,
$$

where $D \rho / D t=\partial \rho / \partial t+\mathbf{u} \cdot \nabla \rho$ is the material derivative, $\nabla$ is the gradient operator and $\nabla \cdot$ is the divergence operator.

conservation of momentum (equation of motion):

$$
\rho \frac{\mathrm{Du}}{\mathrm{Dt}}=-\nabla p-\nabla \cdot \boldsymbol{\tau}+\rho \mathbf{f},
$$

where $\boldsymbol{\tau}$ is the stress tensor and $\mathbf{f}$ is the external force per unit mass of fluid.

conservation of energy (temperature equation):

$$
\rho c_{p} \frac{D T}{D t}=\frac{D p}{D t}-\nabla \cdot \mathbf{q}-\Phi,
$$

where $c_{p}$ is the specific heat at constant pressure, $q$ is the heat flux vector and $\Phi$ is the viscous dissipation function. Appropriate expressions for $\tau, \mathbf{f}, \mathbf{q}$ and $\Phi$ will be given in Section 2.3 and Section 2.4, in correspondence to the chosen space dimension and coordinate system.

The temperature, pressure and density are related via the thermal equation of state, which for ideal gas is

$$
p=\rho R_{m} T,
$$

where $R_{m}$ is the specific gas constant.

\subsection{One-dimensional formulation}

\subsubsection{Governing equations}

Let us consider a one-dimensional region $0<x<\mathrm{L}$, where $\mathrm{L}$ is the length of the tube section of the cooler. We assume

- laminar plug flow,

- Newtonian fluid,

- ideal gas,

- no external forces act on the gas in the tube. 
In the momentum equation (2.2) the viscous stress tensor has now one component, namely

$$
\tau_{x}=-2 \mu \frac{\partial u}{\partial x}+\frac{2}{3} \mu(\nabla \cdot \mathbf{u})=-\frac{4}{3} \mu \frac{\partial u}{\partial x},
$$

where $\mu$ is the dynamic viscosity.

In the energy equation (2.3) the one-dimensional heat flux is

$$
q_{x}=-k_{g} \frac{\partial T}{\partial x}
$$

where $k_{g}$ is the thermal conductivity of the gas. The viscous dissipation term is

$$
\Phi=\tau_{x} \frac{\partial u}{\partial x} .
$$

Substitution of (2.5) - (2.7) in the equations (2.1) - (2.4) gives

$$
\begin{gathered}
\frac{\partial \rho}{\partial t}+\frac{\partial}{\partial x}(\rho u)=0 \\
\rho\left(\frac{\partial u}{\partial t}+u \frac{\partial u}{\partial x}\right)=-\frac{\partial p}{\partial x}+\frac{4}{3} \frac{\partial}{\partial x}\left(\mu \frac{\partial u}{\partial x}\right), \\
\rho c_{p}\left(\frac{\partial T}{\partial t}+u \frac{\partial T}{\partial x}\right)=\frac{\partial p}{\partial t}+u \frac{\partial p}{\partial x}+\frac{\partial}{\partial x}\left(k_{g} \frac{\partial T}{\partial x}\right)+\frac{4}{3} \mu\left(\frac{\partial u}{\partial x}\right)^{2}, \\
p=\rho R_{m} T .
\end{gathered}
$$

The system of equations (2.8) - (2.11) will first be made dimensionless. This leads to identification of the dimensionless numbers that govern the given flow problem. Analysis of dimensionless numbers is highly important and has strong consequences for the resulting set of equations and for the numerical methods to be used.

The scaling parameters are chosen as follows: the shortest physical time-scale of importance is $1 / \omega$ (time for one piston oscillation divided by $2 \pi$ ), where $\omega$ is the angular frequency of the pressure variation. We introduce $\bar{u}$ as the representative amplitude of the velocity variation and therefore $\bar{u} / \omega$ as the typical length-scale. Let $\bar{p}$ be the amplitude of the pressure variation, $p_{a v}$ the average pressure, $T_{a}$ the ambient temperature, $\bar{\rho}$ a typical density, $\bar{\mu}$ a typical viscosity and $\bar{k}_{g}$ a typical thermal conductivity of the gas. We introduce dimensionless variables (indicated by a hat) via

$$
\begin{array}{r}
\rho=\bar{\rho} \hat{\rho}, \quad T=T_{a} \hat{\uparrow}, \quad p=p_{a v}+\bar{p} \hat{p}, \quad u=\bar{u} \hat{u}, \\
x=(\bar{u} / \omega) \hat{x}, \quad t=\hat{t} / \omega, \quad \mu=\bar{\mu} \hat{\mu}, \quad k_{g}=\bar{k}_{g} \hat{k}_{g} .
\end{array}
$$

The way we define the dimensionless pressure differs from the definition of the other dimensionless variables. From the physical model of the pulse tube we know two characteristic values for the driving pressure: the average pressure and the amplitude of the pressure oscillations. It is common [85, p. 580] and, in fact, advantageous 
to work with $p-p_{a v}=\bar{p} \hat{p}$ in place of $p$. This allows us to avoid the problem of cancellation caused by subtracting two nearly equal pressures in the numerical approximation of the pressure gradient.

The governing equations (2.8) - (2.11) become (without ambiguity the hats on the dimensionless variables are now omitted):

$$
\begin{gathered}
\frac{\partial \rho}{\partial t}+\frac{\partial}{\partial x}(\rho u)=0 \\
\rho\left(\frac{\partial u}{\partial t}+u \frac{\partial u}{\partial x}\right)=-\frac{1}{\mathrm{M}^{2}} \frac{\partial p}{\partial x}+\frac{4}{3 \operatorname{Re}} \frac{\partial}{\partial x}\left(\mu \frac{\partial u}{\partial x}\right) \\
\rho\left(\frac{\partial T}{\partial t}+u \frac{\partial T}{\partial x}\right)=\frac{\mathcal{B}(\gamma-1)}{\gamma}\left(\frac{\partial p}{\partial t}+u \frac{\partial p}{\partial x}\right)+\frac{1}{\operatorname{Pe}} \frac{\partial}{\partial x}\left(k_{g} \frac{\partial T}{\partial x}\right)+\frac{4}{3} \mu \frac{\gamma-1}{\gamma} \frac{\mathrm{M}^{2}}{\operatorname{Re}}\left(\frac{\partial u}{\partial x}\right)^{2}, \\
(\mathcal{A}+p) \mathcal{B}=\rho \mathrm{T} .
\end{gathered}
$$

The relevant dimensionless numbers are

$$
\begin{gathered}
\text { oscillatory Reynolds number } \operatorname{Re}=\frac{\bar{\rho} \bar{u}^{2}}{\bar{\mu} \omega}, \\
\text { Prandtl number } \operatorname{Pr}=\frac{c_{p} \bar{\mu}}{k_{g}}, \\
\text { Peclet number } \operatorname{Pe}=\operatorname{Re} \operatorname{Pr}=\frac{\bar{\rho} \bar{u}^{2} c_{p}}{k_{g} \omega}, \\
\text { Mach number } \operatorname{Ma}=\frac{\bar{u}}{\left(p_{a} / \bar{\rho}\right)^{1 / 2}}, \\
\text { modified Mach number } \quad M=\frac{\bar{u}}{(\bar{p} / \bar{\rho})^{1 / 2}}, \\
\text { adiabatic expansion factor } \frac{\gamma-1}{\gamma}=\frac{R_{m}}{c_{p}}, \\
\text { pressure ratio } \mathcal{A}=\frac{p_{a v}}{\bar{p}}, \\
\mathcal{B}=\frac{\bar{p}}{\bar{\rho} R_{m} T_{a}} .
\end{gathered}
$$

The Reynolds number in oscillating flow, sometimes called the kinetic Reynolds number, depends on frequency. It indicates how strong the inertia forces are compared to the viscous forces. The Prandtl number gives an indication for the conduction of heat with respect to the diffusion of momentum. It depends only on the properties of the fluid. The Peclet number shows the ratio of heat convection to heat conduction. The Mach number Ma provides a measure of the compressibility (or change in density) due to motion. It is defined as the ratio of convective velocity to 
acoustic velocity. The dimensionless number $\mathrm{M}$ is a more convenient parameter, the pressure amplitude $\bar{p}$ and not the average pressure $p_{a v}$ is used in the definition of it. $\mathrm{M}$ is related to the Mach number by $\mathrm{M}=\operatorname{Ma} \sqrt{\mathcal{A}}$. With respect to the definition of $\mathrm{M}$ it is important to note that $\bar{u} \rightarrow 0$ if $\bar{p} \rightarrow 0$.

The considerations above show that the basic equations (2.13) - (2.16) contain seven dimensionless parameters: $1 / \mathrm{M}^{2}, 1 / \operatorname{Re},(\gamma-1) / \gamma, 1 / \mathrm{Pe}, \mathrm{M}^{2} / \operatorname{Re}, \mathcal{A}$ and $\mathcal{B}$. If $\bar{\rho}=$ $\mathrm{p}_{\mathrm{av}} / \mathrm{R}_{\mathrm{m}} \mathrm{T}_{\mathrm{a}}$ then $\mathcal{A}$ and $\mathcal{B}$ are related and $\mathcal{B}=1 / \mathcal{A}$.

It is worth mentioning that there are many ways of choosing scales of velocity, time and length to characterise different effects. If the radius of the tube rather than the displacement amplitude is taken as the length scale, different dimensionless numbers appear. People use the Womersley number $\lambda=R_{0} \sqrt{\omega / v}$ or the Valensi number $V a=\omega R_{0}^{2} / v$, where $R_{0}$ is the tube radius and $v$ is the kinematic viscosity, to characterise oscillating flow. The Valensi number and the Womersley number are trivially related, each of which is sometimes called Stokes parameter. Acoustic effects become important at a time scale much smaller than $1 / \omega$. Taking $L /\left(p_{a v} / \bar{\rho}\right)^{1 / 2}$ as the time scale and $L$ as the length scale in the non-dimensional analysis leads to acoustic $1 / \mathrm{Ma}$ terms in the equations (2.13) - (2.16).

The onset of turbulence in oscillating flow has been given a great deal of attention. At present there is a wealth of experimental and phenomenological information in the literature: from the early studies [25], [50], [61] to the more recent investigations [9], [13], [40], [57], [63], [70], [92]. The experimental results show that the onset of turbulence in oscillating flow is different from that in quasi-steady flow. The critical transition parameter, identified in [40], is a Reynolds number based on the Stokes layer thickness $\delta$,

$$
\operatorname{Re}_{\delta}=\frac{\bar{u} \delta}{v}, \delta=\sqrt{\frac{2 v}{\omega}} .
$$

Brereton and Mankbadi [9] summarised experimental results and defined the stability plane for oscillating pipe flow in the $\mathrm{Re}_{\delta}$ versus $R_{0} / \delta$ space. The approximate subdivision of this two-parameter space, as given in [9] , is shown in Figure 2.4. According to this figure, turbulence occurs if $\operatorname{Re}_{\delta}>500$.

Four regimes are to be distinguished:

1. entirely laminar flow,

2. perturbed laminar flow,

3. intermittently turbulent flow,

4. fully turbulent flow.

In the case of laminar flow the cross-sectional distribution of the axial velocity gradually varies in shape with an increase in the ratio $R_{0} / \delta$. It starts from the parabolic shape, as in steady flow, and evolves to a rectangular-like shape. As $R_{0} / \delta$ increases, the maximum flow velocity appears near the pipe wall. In perturbed laminar flow small amplitude perturbations appear either in the accelerating phase near the central axis 


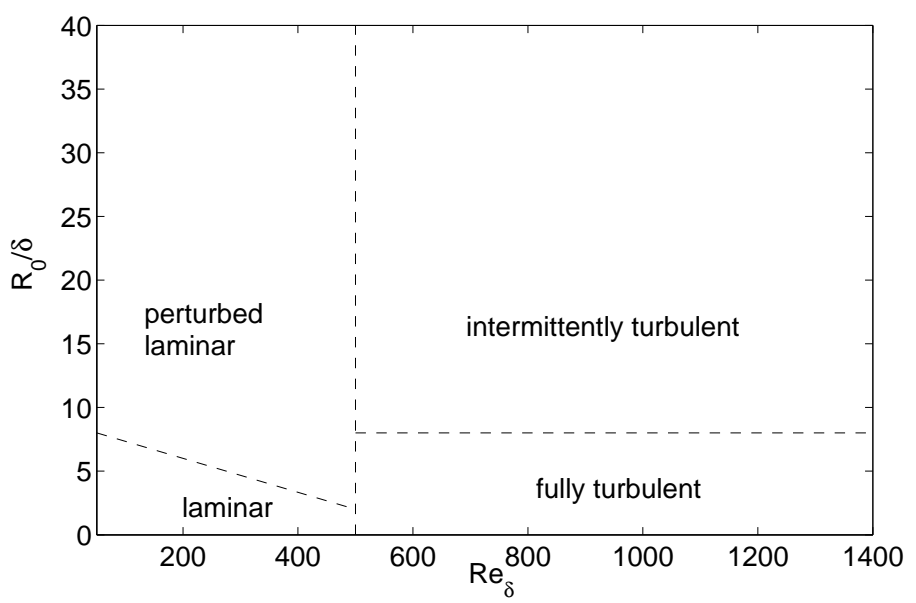

Figure 2.4: Oscillating flow regimes.

of the pipe or near the wall. In intermittently turbulent flow turbulent-like activity appears near the walls during periods of deceleration. It was found experimentally, using smoke visualisation and a hot wire anemometer [92], that at high kinetic Reynolds numbers periodic turbulent bursts occur near the wall. A laminar-like flow exists during the acceleration phase of the cycle, whereas a turbulent-like flow exists during a certain period of the deceleration phase. This is because at high kinetic Reynolds numbers the axial velocity near the wall is higher than at the centerline such that there exists an inflexion point in the velocity profile near the wall. This state has been termed turbulescent by some researchers. In fully turbulent flow turbulent bursts occur in the acceleration phase as well as in the deceleration phase.

Let us consider the pulse tube operating at $20 \mathrm{~Hz}$. For such tubes the velocity amplitude is $1.5 \mathrm{~m} / \mathrm{s}$. The average distance which gas particles travel in the tube during one-half of an oscillating cycle (displacement length) is $0.012 \mathrm{~m}$. The length of the tube is $0.2 \mathrm{~m}$. All relevant physical data for a typical single-inlet pulse tube, operating at $20 \mathrm{~Hz}$, are given in Appendix A. The corresponding dimensionless numbers are

$$
\begin{gathered}
\operatorname{Re} \sim 4.2 \times 10^{3}, \quad \operatorname{Pr} \sim 0.66, \quad \operatorname{Pe} \sim 2.6 \times 10^{3}, \quad \mathrm{Ma} \sim 1.9 \times 10^{-3}, \\
\mathrm{M} \sim 4.6 \times 10^{-3}, \frac{\gamma-1}{\gamma} \sim 0.4, \mathcal{A} \sim 6, \mathcal{B} \sim 0.17 .
\end{gathered}
$$

For the given pulse tube parameters, we have $R_{0} / \delta \approx 96, R_{\delta} \approx 230$, which is in the perturbed-laminar flow region in Figure 2.4. 


\subsubsection{Low-Mach-number approximation}

In our pulse tube, specified above, the Mach number is small. Although compressibility cannot be neglected because of density changes induced by the driving pressure. This type of flow is called low-Mach-number or weakly compressible flow.

The momentum equation (2.14) can be rewritten as follows

$$
\frac{\partial p}{\partial x}=\frac{4 M^{2}}{3 \operatorname{Re}} \frac{\partial}{\partial x}\left(\mu \frac{\partial u}{\partial x}\right)-M^{2} \rho\left(\frac{\partial u}{\partial t}+u \frac{\partial u}{\partial x}\right) .
$$

The first and second terms on the right represent viscous and inertial forces. For example, in the specified pulse tube the constants $\mathrm{M}^{2} / \mathrm{Re}$ and $\mathrm{M}^{2}$ are of the order of $10^{-9}$ and $10^{-5}$, respectively. This means that the inertial and viscous forces are too small to produce a significant pressure gradient; so the right-hand side of equation (2.17) is approximately zero.

Mathematically, as $\mathrm{M}^{2}$ tends to zero, the pressure gradient contribution in the momentum equation (2.14) tends to zero. To explore the consequences of this, we perform an asymptotic analysis, closely following [37]. We expand the four relevant gas dynamic variables in terms of powers of $\mathrm{M}^{2}$. The variables in series form are

$$
\begin{aligned}
& p(x, t)=p_{0}(x, t)+M^{2} p_{1}(x, t)+o\left(M^{2}\right), \\
& u(x, t)=u_{0}(x, t)+M^{2} u_{1}(x, t)+o\left(M^{2}\right), \\
& \rho(x, t)=\rho_{0}(x, t)+M^{2} \rho_{1}(x, t)+o\left(M^{2}\right), \\
& T(x, t)=T_{0}(x, t)+M^{2} T_{1}(x, t)+o\left(M^{2}\right) .
\end{aligned}
$$

Substituting the expansions (2.18) - (2.21) into the original system (2.13) - (2.16), combining all powers of $\mathrm{M}^{2}$ and equating them to zero, yields the following two systems:

leading-order system

$$
\begin{gathered}
\frac{\partial \rho_{0}}{\partial t}+\frac{\partial}{\partial x}\left(\rho_{0} u_{0}\right)=0 \\
\frac{\partial p_{0}}{\partial x}=0 \\
\rho_{0}\left(\frac{\partial T_{0}}{\partial t}+u_{0} \frac{\partial T_{0}}{\partial x}\right)=\frac{\mathcal{B}(\gamma-1)}{\gamma} \frac{\partial p_{0}}{\partial t}+\frac{1}{P e} \frac{\partial}{\partial x}\left(k_{g} \frac{\partial T_{0}}{\partial x}\right), \\
\left(\mathcal{A}+p_{0}\right) \mathcal{B}=\rho_{0} T_{0} .
\end{gathered}
$$

first-order system

$$
\begin{gathered}
\frac{\partial \rho_{1}}{\partial t}+\frac{\partial}{\partial x}\left(\rho_{1} u_{0}\right)+\frac{\partial}{\partial x}\left(\rho_{0} u_{1}\right)=0 \\
\rho_{0}\left(\frac{\partial u_{0}}{\partial t}+u_{0} \frac{\partial u_{0}}{\partial x}\right)=-\frac{\partial p_{1}}{\partial x}+\frac{4}{3 \operatorname{Re}} \frac{\partial}{\partial x}\left(\mu \frac{\partial u_{0}}{\partial x}\right),
\end{gathered}
$$




$$
\begin{gathered}
\rho_{0}\left(\frac{\partial \mathrm{T}_{1}}{\partial t}+\mathrm{u}_{0} \frac{\partial \mathrm{T}_{1}}{\partial x}\right)+\rho_{1} \frac{\partial \mathrm{T}_{0}}{\partial t}+\left(\rho_{0} u_{1}+\rho_{1} u_{0}\right) \frac{\partial \mathrm{T}_{0}}{\partial x}=\frac{\mathcal{B}(\gamma-1)}{\gamma}\left(\frac{\partial p_{1}}{\partial t}+u_{0} \frac{\partial p_{1}}{\partial x}\right)+ \\
\frac{1}{\operatorname{Pe}} \frac{\partial}{\partial x}\left(k_{\mathrm{g}} \frac{\partial \mathrm{T}_{1}}{\partial x}\right)+\frac{4}{3} \mu \frac{\gamma-1}{\gamma} \frac{1}{\operatorname{Re}}\left(\frac{\partial u_{0}}{\partial x}\right)^{2}, \\
p_{1} \mathcal{B}=\rho_{0} \mathrm{~T}_{1}+\rho_{1} \mathrm{~T}_{0}
\end{gathered}
$$

The asymptotic consideration reveals several key features of low-Mach-number flows.

First, it exhibits two physically distinct roles of pressure, namely as

- a thermodynamic variable, $p_{0}$, and as

- a hydrodynamic variable, $p_{1}$.

The acoustic pressure, which is $\mathcal{O}(\mathrm{M})$, does not play a role in our system. It is excluded from the analysis by choosing the time scale as $1 / \omega$. In this sense, the name pulse tube is misleading.

Equation (2.23) shows that the leading pressure is spatially uniform and, therefore, a function of time only. We shall refer to $p_{0}$ as the "thermodynamic" pressure and denote it by $P(t)$. It appears in the temperature equation (2.24) and in the equation of state (2.25). $P(t)$ is the global pressure driving the system. Herein it is a given function of time.

Second, it shows that the contribution of the viscous forces to the energy equation, the last term in the right-hand side of (2.15), is not in the leading order system. It appears for the first time in the energy equation (2.28) of order $\mathcal{O}\left(\mathrm{M}^{2}\right)$.

Neglecting the trivial momentum equation and dropping the zero subscript, the leading-order system (2.22) - (2.25) simplifies to

$$
\begin{gathered}
\frac{\partial \rho}{\partial t}+\frac{\partial}{\partial x}(\rho u)=0 \\
\rho\left(\frac{\partial T}{\partial t}+u \frac{\partial T}{\partial x}\right)=\frac{\mathcal{B}(\gamma-1)}{\gamma} \frac{d P}{d t}+\frac{1}{\operatorname{Pe}} \frac{\partial}{\partial x}\left(k_{g} \frac{\partial T}{\partial x}\right), \\
(\mathcal{A}+\mathrm{P}) \mathcal{B}=\rho \mathrm{T} .
\end{gathered}
$$

The system (2.30) - (2.32) governs the three unknowns $\rho(x, t), u(x, t), T(x, t)$. Combining the equations (2.30) and (2.31), we arrive at

$$
\frac{\partial(\rho \mathrm{T})}{\partial t}+\frac{\partial(\rho u T)}{\partial x}=\frac{\mathcal{B}(\gamma-1)}{\gamma} \frac{d P}{d t}+\frac{1}{P e} \frac{\partial}{\partial x}\left(k_{g} \frac{\partial T}{\partial x}\right)
$$

Substitution of (2.32) in the left-hand side of (2.33) eliminates $\rho$,

$$
\frac{\mathrm{d}((\mathcal{A}+\mathrm{P}) \mathcal{B})}{\mathrm{dt}}+\frac{\partial((\mathcal{A}+\mathrm{P}) \mathcal{B} u)}{\partial x}=\frac{\mathcal{B}(\gamma-1)}{\gamma} \frac{\mathrm{dP}}{\mathrm{dt}}+\frac{1}{\mathrm{Pe}} \frac{\partial}{\partial x}\left(k_{\mathrm{g}} \frac{\partial \mathrm{T}}{\partial x}\right) .
$$


We thus find the following equation for the velocity

$$
\frac{\partial u}{\partial x}=-\frac{1}{(\mathcal{A}+\mathrm{P}) \gamma} \frac{\mathrm{dP}}{\mathrm{dt}}+\frac{1}{\mathcal{B}(\mathcal{A}+\mathrm{P})} \frac{1}{\mathrm{Pe}} \frac{\partial}{\partial x}\left(k_{\mathrm{g}} \frac{\partial \mathrm{T}}{\partial \mathrm{x}}\right) .
$$

To obtain the equation for the temperature, we eliminate the density in (2.31), using equation (2.32). Thus

$$
\frac{(\mathcal{A}+\mathrm{P}) \mathcal{B}}{\mathrm{T}}\left(\frac{\partial \mathrm{T}}{\partial \mathrm{t}}+\mathrm{u} \frac{\partial \mathrm{T}}{\partial x}\right)=\frac{\mathcal{B}(\gamma-1)}{\gamma} \frac{\mathrm{dP}}{\mathrm{dt}}+\frac{1}{\mathrm{Pe}} \frac{\partial}{\partial x}\left(k_{\mathrm{g}} \frac{\partial \mathrm{T}}{\partial x}\right) .
$$

Finally the equations for the velocity and the temperature are

$$
\begin{gathered}
\frac{\partial u}{\partial x}=\epsilon(t) \frac{\partial}{\partial x}\left(k_{g} \frac{\partial T}{\partial x}\right)+s_{1}(t), \\
\frac{\partial T}{\partial t}=\epsilon(t) T \frac{\partial}{\partial x}\left(k_{g} \frac{\partial T}{\partial x}\right)-u \frac{\partial T}{\partial x}+s_{2}(t) T
\end{gathered}
$$

where

$$
\begin{aligned}
\mathrm{s}_{1}(\mathrm{t}) & =-\frac{1}{(\mathcal{A}+\mathrm{P}(\mathrm{t})) \gamma} \frac{\mathrm{dP}(\mathrm{t})}{\mathrm{dt}}, \\
\mathrm{s}_{2}(\mathrm{t}) & =\frac{1}{(\mathcal{A}+\mathrm{P}(\mathrm{t}))} \frac{\gamma-1}{\gamma} \frac{\mathrm{dP}(\mathrm{t})}{\mathrm{dt}}, \\
\epsilon(\mathrm{t}) & =\frac{1}{\mathcal{B}(\mathcal{A}+\mathrm{P}(\mathrm{t}))} \frac{1}{\mathrm{Pe}} \ll 1 .
\end{aligned}
$$

Assuming that the gas thermal conductivity $k_{g}$ is constant (in terms of dimensionless values this means that $\left.\hat{k}_{g}=1\right),(2.37),(2.38)$ simplify to

$$
\begin{gathered}
\frac{\partial u}{\partial x}=\epsilon(t) \frac{\partial^{2} T}{\partial x^{2}}+s_{1}(t), \\
\frac{\partial T}{\partial t}=\epsilon(t) T \frac{\partial^{2} T}{\partial x^{2}}-u \frac{\partial T}{\partial x}+s_{2}(t) T .
\end{gathered}
$$

The equations (2.42), (2.43) form the basis of our one-dimensional model. Note that (2.42) is a velocity divergence constraint. We observe that the velocity divergence is driven by heat conduction and global pressure changes. The temperature equation (2.43) is a nonlinear convection-diffusion equation with the presence of convection by the variable velocity $u(x, t)$ and diffusion through the diffusion coefficient $\epsilon T$, see (2.41). The diffusion coefficient is denoted by $\epsilon \mathrm{T}$ in order to emphasise that, according to our dimensional analysis, it has a small value.

\subsubsection{Boundary and initial conditions}

To complete the mathematical model, we define boundary and initial conditions. The position $x=0$ is the hot end of the tube and $x=L$ is the cold end. 


\section{Orifice pulse-tube refrigerator}

We need only one boundary condition for the velocity in equation (2.37) and we choose the velocity at the hot end $u_{H}(t)$. To find the velocity at the hot end in the orifice pulse tube, Figure $2.1 \mathrm{~b}$, we consider the volume flow $\dot{V}_{\mathrm{H}}$ through the orifice, which is assumed to satisfy the linear resistance law

$$
\dot{\mathrm{V}}_{\mathrm{H}}(\mathrm{t})=-\mathrm{C}_{\mathrm{or}}\left(\mathrm{p}_{\mathrm{t}}(\mathrm{t})-\mathrm{p}_{\mathrm{b}}(\mathrm{t})\right)
$$

where $p_{t}(t)$ is the tube pressure, $p_{b}(t)$ is the pressure in the buffer and $C_{o r}$ is the flow conductance of the orifice. The velocity is then given by

$$
u(0, t)=u_{H}(t)=-\frac{C_{o r}}{A_{t}}\left(p_{t}(t)-p_{b}(t)\right)
$$

where $A_{t}$ is the cross-sectional area of the tube.

Special care has been taken in defining the buffer pressure $p_{b}(t)$ in boundary condition (2.45). If the buffer volume is large enough, then the buffer pressure is constant and the following approximation, based on the first-order harmonic analysis, can be used, see [66, p. 61],

$$
p_{b}=\sqrt{p_{a v}^{2}+\frac{\bar{p}^{2}}{2}} .
$$

A time-dependent buffer pressure $p_{b}(t)$ leads to a more realistic boundary condition for the velocity, such that the cycle-averaged mass flow through the hot end finally becomes zero. The volume flow through the orifice causes adiabatic compression/expansion in the buffer according to the thermodynamic Poisson law. This gives

$$
\dot{V}_{\mathrm{H}}(\mathrm{t})=-\frac{\mathrm{c}_{v}}{\mathrm{c}_{\mathrm{p}}} \frac{\mathrm{V}_{\mathrm{b}}}{\mathrm{p}_{\mathrm{b}}(\mathrm{t})} \frac{\mathrm{d} \mathrm{p}_{\mathrm{b}}(\mathrm{t})}{\mathrm{dt}} .
$$

Combining (2.44) and (2.47), we obtain the following ordinary differential equation for the buffer pressure

$$
\frac{d p_{b}}{d t}=\frac{C_{o r} c_{p}}{V_{b} c_{v}} p_{b}(t)\left(p_{t}(t)-p_{b}(t)\right)
$$

For the derived boundary condition (2.45) and the buffer pressure equation (2.48) we perform the non-dimensionalisation and asymptotic consideration from the previous sections. By taking $p_{t}(t)=p_{a v}+\bar{p} \hat{p}(t)$ and $p_{b}(t)=\bar{p}_{b} \hat{p}_{b}(t)$, the dimensionless form of the boundary condition for the leading-order velocity is (omitting hats)

$$
u_{H}(t)=-\frac{C_{o r}}{A_{t}} \frac{\bar{p}}{\bar{u}}\left(P(t)+\frac{p_{a v}}{\bar{p}}-\frac{\bar{p}_{b}}{\bar{p}} p_{b}(t)\right),
$$

or

$$
\mathrm{u}_{\mathrm{H}}(\mathrm{t})=-\mathcal{C}\left(\mathrm{P}(\mathrm{t})+\mathcal{A}-\mathcal{E} \mathrm{p}_{\mathrm{b}}(\mathrm{t})\right)
$$

with 


$$
\mathcal{C}=\frac{C_{\text {or }}}{A_{\mathrm{t}}} \frac{\bar{p}}{\overline{\mathrm{u}}}, \mathcal{A}=\frac{p_{\mathrm{a} v}}{\bar{p}}, \mathcal{E}=\frac{\bar{p}_{\mathrm{b}}}{\bar{p}} .
$$

The dimensionless equation (2.48) reads

$$
\left\{\begin{array}{l}
\frac{d p_{b}}{d t}=\mathcal{D} p_{b}(t)\left(P(t)+\mathcal{A}-\mathcal{E} p_{b}(t)\right) \\
p_{b}(0)=1
\end{array}\right.
$$

with

$$
\mathcal{D}=\frac{\mathrm{C}_{\mathrm{or}} \mathrm{c}_{\mathrm{p}}}{\mathrm{V}_{\mathrm{b}} \mathrm{c}_{v}} \frac{\overline{\mathrm{p}}}{\omega} .
$$

The nonlinear differential equation (2.52) is known as a Riccati equation. It can sometimes be solved analytically if an analytical expression for the shape of the pressure oscillation $\mathrm{P}(\mathrm{t})$ is given (for example a sinusoidal function). In other cases, numerical methods should be used.

The temperature equation (2.38) requires two boundary conditions and an initial condition. Because the flow in the tube reverses periodically, the boundary conditions for the temperature at any moment of time depend on the local velocity direction. If the gas is flowing into the tube, it has the temperature of the heat exchanger ( $T_{H}$ at the hot end and $T_{C}$ at the cold end). If the gas is flowing out of the tube, it never has the temperature of the heat exchanger. If we assume a zero heat flux at the boundary it will give us homogeneous Neuman boundary conditions. Another and better possibility would be to use the temperature equation (2.38) at the boundaries to approximately compute the heat flux. Then the homogeneous Neuman boundary conditions are replaced by

$$
\begin{aligned}
& u(0, t) \frac{\partial T}{\partial x}(0, t)=s_{2}(t) T(0, t)-\frac{\partial T}{\partial t}(0, t), \\
& u(L, t) \frac{\partial T}{\partial x}(L, t)=s_{2}(t) T(L, t)-\frac{\partial T}{\partial t}(L, t) .
\end{aligned}
$$

The boundary conditions are thus

$$
\begin{aligned}
& \left\{\begin{array}{l}
T(0, t)=T_{H} \text { if } u(0, t) \geq 0 \\
\frac{\partial T}{\partial x}(0, t)=\left(s_{2}(t) T(0, t)-\frac{\partial T}{\partial t}(0, t)\right) / u(0, t) \text { if } u(0, t)<0
\end{array}\right. \\
& \left\{\begin{array}{l}
T(L, t)=T_{C} \text { if } u(L, t) \leq 0 \\
\frac{\partial T}{\partial x}(L, t)=\left(s_{2}(t) T(L, t)-\frac{\partial T}{\partial t}(L, t)\right) / u(L, t) \text { if } u(L, t)>0
\end{array}\right.
\end{aligned}
$$

Note that with "upwinding" the BC's we avoid the problem of the tiny $(\varepsilon \ll 1)$ thermal boundary layers near the heat exchangers. 
A linear profile can be taken as initial condition for the temperature

$$
\mathrm{T}(\mathrm{x}, 0)=\mathrm{T}_{\mathrm{H}}-\left(\mathrm{T}_{\mathrm{H}}-\mathrm{T}_{\mathrm{C}}\right) \frac{\mathrm{x}}{\mathrm{L}} \text {. }
$$

The corresponding velocity is then obtained from (2.42) as

$$
u(x, 0)=s_{1}(0) x+u_{H}(0)
$$

\section{Basic pulse-tube refrigerator}

For the basic pulse tube, Fig. 2.1a, the velocity at the hot end $u_{H}(t)$ is zero. The hot end temperature is constant and the cold end temperature varies with velocity, like (2.55)

$$
\left\{\begin{array}{l}
T(0, t)=T_{H} \\
T(L, t)=T_{C} \text { if } u(L, t) \leq 0 \\
u(L, t) \frac{\partial T}{\partial x}(L, t)=s_{2}(t) T(L, t)-\frac{\partial T}{\partial t}(L, t) \text { if } u(L, t)>0
\end{array} .\right.
$$

\subsubsection{Two-dimensional corrections to the one-dimensional model}

The tube wall plays an important role in the cooling process. Wall effects can be included in our one-dimensional model. Additional terms will appear in the momentum equation (friction force at the wall) and in the energy equation (heat transfer with the wall). The modified momentum and energy equations (2.9), (2.10) are

$$
\begin{gathered}
\rho\left(\frac{\partial u}{\partial t}+u \frac{\partial u}{\partial x}\right)=-\frac{\partial p}{\partial x}+\frac{4}{3} \frac{\partial}{\partial x}\left(\mu \frac{\partial u}{\partial x}\right)-c_{f} u \\
\rho c_{p}\left(\frac{\partial T}{\partial t}+u \frac{\partial T}{\partial x}\right)=\frac{\partial p}{\partial t}+u \frac{\partial p}{\partial x}+\frac{\partial}{\partial x}\left(k_{g} \frac{\partial T}{\partial x}\right)+\frac{4}{3} \mu\left(\frac{\partial u}{\partial x}\right)^{2}+h\left(T_{w}-T\right),
\end{gathered}
$$

where $c_{f}$ is the friction factor, $h$ is the convective heat transfer coefficient and $T_{w}$ is the temperature of the wall.

Because of lack of suitable heat transfer and pressure drop correlations for reciprocating flow conditions, the modelling is usually based on correlations derived from steady-state unidirectional laminar flow assumptions, see [32, p. 490]. Thus quasisteady models use

$$
\begin{gathered}
\mathrm{c}_{\mathrm{f}}=8 \mu / \mathrm{R}_{0}^{2}, \\
\mathrm{~h}=\frac{\mathrm{Nu_{ \textrm {D } } \mathrm { k } _ { \mathrm { g } }}}{\mathrm{D}} \text { with } \mathrm{Nu}_{\mathrm{D}}=3.66 .
\end{gathered}
$$

However, this approach proved to be insufficient for the analysis of momentum and heat transfer in unsteady oscillating flows at high frequencies.

The available experimental data for the friction factor and the convective heat transfer coefficient for reciprocating flow can be summarised as follows: 
- The friction factor is larger than in steady flow. In [96] the additional term that accounts for unsteady friction was derived. This term, which depends on the flow history, can be incorporated in a numerical model. Experimental results for fully developed reciprocating laminar flow are given in [93]. For hydrodynamically developing reciprocating laminar-turbulent flow relatively few papers have been reported, see [31].

- The convective heat transfer coefficient is much larger than in steady flow. The heat transfer of working gas can be enhanced by the flow oscillations at rates orders of magnitude greater than by pure conduction, see $[38,39,94]$. Mechanisms of this enhancement are not yet explained. Even though such mechanisms have been proposed in recent literature [41,45], they are not all in agreement. Experimental data available for the heat transfer correlations for developing laminar reciprocating flow are limited and quite incomplete and therefore not suitable for general use.

From the above considerations it can be concluded that for an accurate description of fluid-wall interaction in oscillating flow one has to apply two dimensional modelling.

\subsection{Two-dimensional formulation}

\subsubsection{Governing equations}

The two-dimensional axisymmetrical domain under consideration is shown in Figure 2.5. Gas flows in the cylindrical tube of length $L$ and inner radius $R_{0}$. The tube wall has thickness $l=R_{1}-R_{0}$. Assuming the fluid to be Newtonian and neglecting external forces, the equations (2.1) - (2.4) in cylindrical axisymmetrical coordinates, see [8], are

$$
\begin{gathered}
\frac{\partial \rho}{\partial t}+\frac{\partial}{\partial z}(\rho u)+\frac{1}{r} \frac{\partial}{\partial r}(r \rho v)=0 \\
\rho\left(\frac{\partial u}{\partial t}+u \frac{\partial u}{\partial z}+v \frac{\partial u}{\partial r}\right)=-\frac{\partial p}{\partial z}-\left[\frac{1}{r} \frac{\partial}{\partial r}\left(r \tau_{r z}\right)+\frac{\partial \tau_{z z}}{\partial z}\right] \\
\rho\left(\frac{\partial v}{\partial t}+u \frac{\partial v}{\partial z}+v \frac{\partial v}{\partial r}\right)=-\frac{\partial p}{\partial r}-\left[\frac{1}{r} \frac{\partial}{\partial r}\left(r \tau_{r r}\right)+\frac{\partial \tau_{r z}}{\partial z}\right] \\
\rho c_{p}\left(\frac{\partial T}{\partial t}+u \frac{\partial T}{\partial z}+v \frac{\partial T}{\partial r}\right)=\frac{\partial p}{\partial t}+u \frac{\partial p}{\partial z}+v \frac{\partial p}{\partial r}+\frac{1}{r} \frac{\partial}{\partial r}\left(r k_{g} \frac{\partial T}{\partial r}\right)+\frac{\partial}{\partial z}\left(k_{g} \frac{\partial T}{\partial z}\right)+\Phi \\
p=\rho R_{m} T
\end{gathered}
$$

The components of the viscous stress tensor in the equations (2.64) and (2.65) are

$$
\tau_{z z}=-2 \mu \frac{\partial u}{\partial z}+\frac{2}{3} \mu(\nabla \cdot \mathbf{u})=-\mu\left[\frac{4}{3} \frac{\partial u}{\partial z}-\frac{2}{3} \frac{v}{r}-\frac{2}{3} \frac{\partial v}{\partial r}\right],
$$




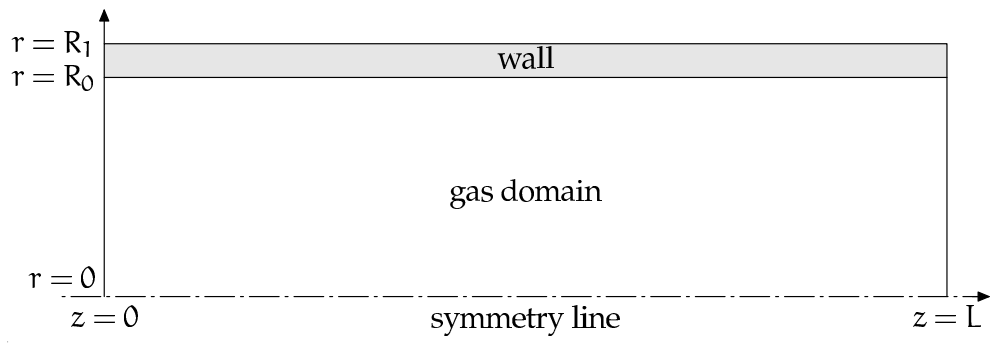

Figure 2.5: Two-dimensional axisymmetrical domain.

$$
\begin{gathered}
\tau_{r r}=-2 \mu \frac{\partial v}{\partial r}+\frac{2}{3} \mu(\nabla \cdot \mathbf{u})=-\mu\left[\frac{4}{3} \frac{\partial v}{\partial r}-\frac{2}{3} \frac{v}{r}-\frac{2}{3} \frac{\partial u}{\partial z}\right], \\
\tau_{r z}=-\mu\left(\frac{\partial u}{\partial r}+\frac{\partial v}{\partial z}\right), \\
\nabla \cdot \mathbf{u}=\frac{1}{r} \frac{\partial}{\partial r}(r v)+\frac{\partial u}{\partial z} .
\end{gathered}
$$

The viscous dissipation factor in cylindrical axisymmetrical coordinates is

$$
\Phi=-(\tau: \nabla \cdot \mathbf{u})=-\tau_{z z} \frac{\partial u}{\partial z}-\tau_{r r} \frac{\partial v}{\partial r}-\tau_{r z}\left(\frac{\partial u}{\partial r}+\frac{\partial v}{\partial z}\right) .
$$

Dimensionless variables are introduced as in the one-dimensional case

$$
\begin{aligned}
& \rho=\bar{\rho} \hat{\rho}, T=T_{a} \hat{\top}, \quad p=p_{a v}+\bar{p} \hat{p}, \quad t=\hat{t} / \omega, k_{g}=\bar{k}_{g} \hat{k}_{g}, \mu=\bar{\mu} \hat{\mu}, \\
& u=\bar{u} \hat{u}, \quad v=\bar{v} \hat{v}, \quad z=(\bar{u} / \omega) \hat{z}, \quad r=(\bar{v} / \omega) \hat{r}, \bar{u}=\bar{v} .
\end{aligned}
$$

The typical velocities and length scales are taken the same in axial and radial directions. We like to have a general model valid for any tube, without the assumption that the tube length is much larger than the tube radius. This gives us the possibility to study entrance effects in the tubes with arbitrary L/D ratios.

After non-dimensionalisation the governing equations (2.63) - (2.67) become

$$
\begin{gathered}
\frac{\partial \rho}{\partial t}+\frac{\partial}{\partial z}(\rho u)+\frac{1}{r} \frac{\partial}{\partial r}(r \rho v)=0 \\
\rho\left(\frac{\partial u}{\partial t}+u \frac{\partial u}{\partial z}+v \frac{\partial u}{\partial r}\right)=-\frac{1}{M^{2}} \frac{\partial p}{\partial z}-\frac{1}{\operatorname{Re}}\left[\frac{1}{r} \frac{\partial}{\partial r}\left(r \tau_{r z}\right)+\frac{\partial \tau_{z z}}{\partial z}\right] \\
\rho\left(\frac{\partial v}{\partial t}+u \frac{\partial v}{\partial z}+v \frac{\partial v}{\partial r}\right)=-\frac{1}{M^{2}} \frac{\partial p}{\partial r}-\frac{1}{\operatorname{Re}}\left[\frac{1}{r} \frac{\partial}{\partial r}\left(r \tau_{r r}\right)+\frac{\partial \tau_{r z}}{\partial z}\right]
\end{gathered}
$$




$$
\begin{array}{r}
\rho\left(\frac{\partial T}{\partial t}+u \frac{\partial T}{\partial z}+v \frac{\partial T}{\partial r}\right)=\frac{\mathcal{B}(\gamma-1)}{\gamma}\left(\frac{\partial p}{\partial t}+u \frac{\partial p}{\partial z}+v \frac{\partial p}{\partial r}\right)+\frac{1}{\operatorname{Pe}} \frac{1}{r} \frac{\partial}{\partial r}\left(r k_{g} \frac{\partial T}{\partial r}\right)+ \\
\frac{1}{\operatorname{Pe}} \frac{\partial}{\partial z}\left(k_{g} \frac{\partial T}{\partial z}\right)+\frac{\gamma-1}{\gamma} \frac{M^{2}}{\operatorname{Re}} \Phi
\end{array}
$$

$$
(\mathcal{A}+\mathrm{p}) \mathcal{B}=\rho \mathrm{T} .
$$

\subsubsection{Low-Mach-number approximation}

Closely following the procedure from the one-dimensional consideration, we expand the variables in terms of powers of $\mathrm{M}^{2}$. The variables are written now in series form as

$$
\begin{aligned}
& p(z, r, t)=p_{0}(z, r, t)+M^{2} p_{1}(z, r, t)+o\left(M^{2}\right), \\
& u(z, r, t)=u_{0}(z, r, t)+M^{2} u_{1}(z, r, t)+o\left(M^{2}\right), \\
& v(z, r, t)=v_{0}(z, r, t)+M^{2} v_{1}(z, r, t)+o\left(M^{2}\right), \\
& \rho(z, r, t)=\rho_{0}(z, r, t)+M^{2} \rho_{1}(z, r, t)+o\left(M^{2}\right), \\
& T(z, r, t)=T_{0}(z, r, t)+M^{2} T_{1}(z, r, t)+o\left(M^{2}\right) .
\end{aligned}
$$

The low-Mach-number approximation replaces the original system (2.73) - (2.77) with the leading order system:

$$
\begin{aligned}
\frac{\partial \rho_{0}}{\partial t}+\frac{\partial}{\partial z}\left(\rho_{0} u_{0}\right)+\frac{1}{r} \frac{\partial}{\partial r}\left(r \rho_{0} v_{0}\right)=0 \\
\frac{\partial p_{0}}{\partial z}=0 \\
\frac{\partial p_{0}}{\partial r}=0 \\
\rho_{0}\left(\frac{\partial T_{0}}{\partial t}+u_{0} \frac{\partial T_{0}}{\partial z}+v_{0} \frac{\partial T_{0}}{\partial r}\right)=\frac{\mathcal{B}(\gamma-1)}{\gamma} \frac{d p_{0}}{d t}+\frac{1}{\operatorname{Pe}}\left[\frac{1}{r} \frac{\partial}{\partial r}\left(r k_{g} \frac{\partial T_{0}}{\partial r}\right)+\frac{\partial}{\partial z}\left(k_{g} \frac{\partial T_{0}}{\partial z}\right)\right] \\
\left(\mathcal{A}+p_{0}\right) \mathcal{B}=\rho_{0} T_{0}
\end{aligned}
$$

As in the one-dimensional case, the equations (2.84), (2.85) show that the leadingorder pressure $p_{0}$ is spatially uniform and therefore a function of time only. Using the notation $p_{0}(z, r, t)=P(t)$, as in Section 2.3.1, the leading-order system reduces to

$$
\begin{gathered}
\frac{\partial \rho_{0}}{\partial t}+\frac{\partial}{\partial z}\left(\rho_{0} u_{0}\right)+\frac{1}{r} \frac{\partial}{\partial r}\left(r \rho_{0} v_{0}\right)=0 \\
\rho_{0}\left(\frac{\partial T_{0}}{\partial t}+u_{0} \frac{\partial T_{0}}{\partial z}+v_{0} \frac{\partial T_{0}}{\partial r}\right)=\frac{\mathcal{B}(\gamma-1)}{\gamma} \frac{d P}{d t}+\frac{1}{P e}\left[\frac{1}{r} \frac{\partial}{\partial r}\left(r k_{g} \frac{\partial T_{0}}{\partial r}\right)+\frac{\partial}{\partial z}\left(k_{g} \frac{\partial T_{0}}{\partial z}\right)\right],
\end{gathered}
$$




$$
(\mathcal{A}+\mathrm{P}) \mathcal{B}=\rho_{0} \mathrm{~T}_{0}
$$

The system (2.88) - (2.90) does not provide sufficient information for defining a two-directional velocity field. The first-order momentum equations

$$
\begin{aligned}
& \rho_{0}\left(\frac{\partial u_{0}}{\partial t}+u_{0} \frac{\partial u_{0}}{\partial z}+v_{0} \frac{\partial u_{0}}{\partial r}\right)=-\frac{\partial p_{1}}{\partial z}-\frac{1}{\operatorname{Re}}\left[\frac{1}{r} \frac{\partial}{\partial r}\left(r \tau_{r z}\right)+\frac{\partial \tau_{z z}}{\partial z}\right], \\
& \rho_{0}\left(\frac{\partial v_{0}}{\partial t}+u_{0} \frac{\partial v_{0}}{\partial z}+v_{0} \frac{\partial v_{0}}{\partial r}\right)=-\frac{\partial p_{1}}{\partial r}-\frac{1}{\operatorname{Re}}\left[\frac{1}{r} \frac{\partial}{\partial r}\left(r \tau_{r r}\right)+\frac{\partial \tau_{r z}}{\partial z}\right],
\end{aligned}
$$

must therefore be added to complete the system. Equations (2.91) and (2.92) contain the first-order, or "hydrodynamic", pressure $p_{1}$, which is not directly influenced by the compression/expansion represented by (2.90). It affects the leading-order velocity acting as a balance to the inertial and viscous forces in the momentum equation. The final system (without zero subscripts for the leading-order variables and with small letter $p$ for the first-order pressure) reads

$$
\begin{gathered}
\frac{\partial \rho}{\partial t}+\frac{\partial}{\partial z}(\rho u)+\frac{1}{r} \frac{\partial}{\partial r}(r \rho v)=0 \\
\rho\left(\frac{\partial u}{\partial t}+u \frac{\partial u}{\partial z}+v \frac{\partial u}{\partial r}\right)=-\frac{\partial p}{\partial z}-\frac{1}{\operatorname{Re}}\left[\frac{1}{r} \frac{\partial}{\partial r}\left(r \tau_{r z}\right)+\frac{\partial \tau_{z z}}{\partial z}\right] \\
\rho\left(\frac{\partial v}{\partial t}+u \frac{\partial v}{\partial z}+v \frac{\partial v}{\partial r}\right)=-\frac{\partial p}{\partial r}-\frac{1}{\operatorname{Re}}\left[\frac{1}{r} \frac{\partial}{\partial r}\left(r \tau_{r r}\right)+\frac{\partial \tau_{r z}}{\partial z}\right] \\
\rho\left(\frac{\partial T}{\partial t}+u \frac{\partial T}{\partial z}+v \frac{\partial T}{\partial r}\right)=\frac{\mathcal{B}(\gamma-1)}{\gamma} \frac{d P}{d t}+\frac{1}{\operatorname{Pe}}\left[\frac{1}{r} \frac{\partial}{\partial r}\left(r k_{g} \frac{\partial T}{\partial r}\right)+\frac{\partial}{\partial z}\left(k_{g} \frac{\partial T}{\partial z}\right)\right], \\
(\mathcal{A}+\mathrm{P}) \mathcal{B}=\rho T .
\end{gathered}
$$

Using the expressions (2.68) - (2.71) the momentum equations can be rewritten in terms of velocity gradients. Assuming constant viscosity, the equations (2.94) and (2.95) become

$$
\begin{aligned}
& \rho\left(\frac{\partial u}{\partial t}+u \frac{\partial u}{\partial z}+v \frac{\partial u}{\partial r}\right)=-\frac{\partial p}{\partial z}+\frac{1}{\operatorname{Re}}\left[\frac{\partial^{2} u}{\partial r^{2}}+\frac{1}{r} \frac{\partial u}{\partial r}+\frac{4}{3} \frac{\partial^{2} u}{\partial z^{2}}+\frac{1}{3} \frac{1}{r} \frac{\partial v}{\partial z}+\frac{1}{3} \frac{\partial}{\partial z}\left(\frac{\partial v}{\partial r}\right)\right], \\
& \rho\left(\frac{\partial v}{\partial t}+u \frac{\partial v}{\partial z}+v \frac{\partial v}{\partial r}\right)=-\frac{\partial p}{\partial r}+\frac{1}{\operatorname{Re}}\left[\frac{4}{3} \frac{\partial^{2} v}{\partial r^{2}}+\frac{\partial^{2} v}{\partial z^{2}}+\frac{2}{3} \frac{1}{r} \frac{\partial v}{\partial r}-\frac{2}{3} \frac{1}{r} \frac{\partial u}{\partial z}+\frac{1}{3} \frac{\partial}{\partial r}\left(\frac{\partial u}{\partial z}\right)\right] .
\end{aligned}
$$

Combining the equations (2.93), (2.96) and (2.97), a velocity divergence constraint is derived in the following three steps

$$
\frac{\partial(\rho \mathrm{T})}{\partial t}+\frac{\partial(\rho \mathrm{Tu})}{\partial z}+\frac{\partial(\rho \mathrm{T} v)}{\partial r}=\frac{\mathcal{B}(\gamma-1)}{\gamma} \frac{d \mathrm{P}}{\mathrm{dt}}+\frac{1}{\mathrm{Pe}}\left[\frac{1}{r} \frac{\partial}{\partial r}\left(r k_{g} \frac{\partial \mathrm{T}}{\partial r}\right)+\frac{\partial}{\partial z}\left(k_{g} \frac{\partial \mathrm{T}}{\partial z}\right)\right],
$$




$$
\begin{aligned}
& \mathcal{B} \frac{\mathrm{dP}}{\mathrm{dt}}+(\mathcal{A}+\mathrm{P}(\mathrm{t})) \mathcal{B}\left(\frac{\partial u}{\partial z}+\frac{\partial v}{\partial \mathrm{r}}+\frac{v}{\mathrm{r}}\right)=\frac{\mathcal{B}(\gamma-1)}{\gamma} \frac{\mathrm{dP}}{\mathrm{dt}}+ \\
& \frac{1}{\operatorname{Pe}}\left[\frac{1}{r} \frac{\partial}{\partial r}\left(r k_{g} \frac{\partial T}{\partial r}\right)+\frac{\partial}{\partial z}\left(k_{g} \frac{\partial T}{\partial z}\right)\right] \\
& \frac{\partial u}{\partial z}+\frac{\partial v}{\partial r}+\frac{v}{r}=-\frac{1}{\gamma} \frac{1}{(\mathcal{A}+\mathrm{P})} \frac{\mathrm{dP}}{\mathrm{dt}}+\frac{1}{(\mathcal{A}+\mathrm{P}) \mathcal{B}} \frac{1}{\mathrm{Pe}}\left[\frac{1}{\mathrm{r}} \frac{\partial}{\partial \mathrm{r}}\left(\mathrm{rk}_{\mathrm{g}} \frac{\partial \mathrm{T}}{\partial \mathrm{r}}\right)+\frac{\partial}{\partial z}\left(\mathrm{k}_{\mathrm{g}} \frac{\partial \mathrm{T}}{\partial z}\right)\right] .
\end{aligned}
$$

From the equations (2.98), (2.99) and (2.102) the "hydrodynamic" role of the firstorder pressure is clearly seen. It guarantees, like the standard pressure variable in incompressible flows, that the velocity field satisfies an elliptic divergence constraint.

Finally, for the five unknowns $\rho(z, r, t), u(z, r, t), v(z, r, t), T(z, r, t)$ and $p(z, r, t)$, the resulting system of equations is given by

$$
\begin{gathered}
\rho\left(\frac{\partial u}{\partial t}+u \frac{\partial u}{\partial z}+v \frac{\partial u}{\partial r}\right)=-\frac{\partial p}{\partial z}+\frac{1}{\operatorname{Re}}\left[\frac{\partial^{2} u}{\partial r^{2}}+\frac{1}{r} \frac{\partial u}{\partial r}+\frac{4}{3} \frac{\partial^{2} u}{\partial z^{2}}+\frac{1}{3} \frac{1}{r} \frac{\partial v}{\partial r}+\frac{1}{3} \frac{\partial}{\partial z}\left(\frac{\partial v}{\partial r}\right)\right], \\
(2.103) \\
\rho\left(\frac{\partial v}{\partial t}+u \frac{\partial v}{\partial z}+v \frac{\partial v}{\partial r}\right)=-\frac{\partial p}{\partial r}+\frac{1}{\operatorname{Re}}\left[\frac{4}{3} \frac{\partial^{2} v}{\partial r^{2}}+\frac{\partial^{2} v}{\partial z^{2}}+\frac{2}{3} \frac{1}{r} \frac{\partial v}{\partial r}-\frac{2}{3} \frac{1}{r} \frac{\partial u}{\partial z}+\frac{1}{3} \frac{\partial}{\partial r}\left(\frac{\partial u}{\partial z}\right)\right], \\
(2.104) \\
\frac{\partial u}{\partial z}+\frac{\partial v}{\partial r}+\frac{v}{r}=-\frac{1}{\gamma} \frac{1}{(\mathcal{A}+\mathrm{P})} \frac{\mathrm{dP}}{\mathrm{dt}}+\frac{1}{(\mathcal{A}+\mathrm{P}(\mathrm{t})) \mathcal{B}} \frac{1}{\mathrm{Pe}}\left[\frac{1}{r} \frac{\partial}{\partial r}\left(r k_{g} \frac{\partial T}{\partial r}\right)+\frac{\partial}{\partial z}\left(k_{g} \frac{\partial T}{\partial z}\right)\right], \\
\rho\left(\frac{\partial T}{\partial t}+u \frac{\partial T}{\partial z}+v \frac{\partial T}{\partial r}\right)=\frac{\mathcal{B}(\gamma-1)}{\gamma} \frac{\mathrm{dP}}{\mathrm{dt}}+\frac{1}{\mathrm{Pe}}\left[\frac{1}{r} \frac{\partial}{\partial r}\left(r k_{g} \frac{\partial T}{\partial r}\right)+\frac{\partial}{\partial z}\left(k_{g} \frac{\partial T}{\partial z}\right)\right], \\
(\mathcal{A}+\mathrm{P}) \mathcal{B}=\rho \mathrm{T} .
\end{gathered}
$$

Using the expressions (2.39), (2.40) for the source terms and the expression (2.41) for the diffusion coefficient, the equations (2.105) and (2.106) become

$$
\begin{gathered}
\frac{\partial u}{\partial z}+\frac{\partial v}{\partial r}+\frac{v}{r}=\epsilon(t)\left[\frac{1}{r} \frac{\partial}{\partial r}\left(r k_{g} \frac{\partial T}{\partial r}\right)+\frac{\partial}{\partial z}\left(k_{g} \frac{\partial T}{\partial z}\right)\right]+s_{1}(t), \\
\frac{\partial T}{\partial t}+u \frac{\partial T}{\partial z}+v \frac{\partial T}{\partial r}=\epsilon(t) T\left[\frac{1}{r} \frac{\partial}{\partial r}\left(r k_{g} \frac{\partial T}{\partial r}\right)+\frac{\partial}{\partial z}\left(k_{g} \frac{\partial T}{\partial z}\right)\right]+s_{2}(t) T .
\end{gathered}
$$

In (2.109) the density has been eliminated by substituting the equation of state (2.107). 


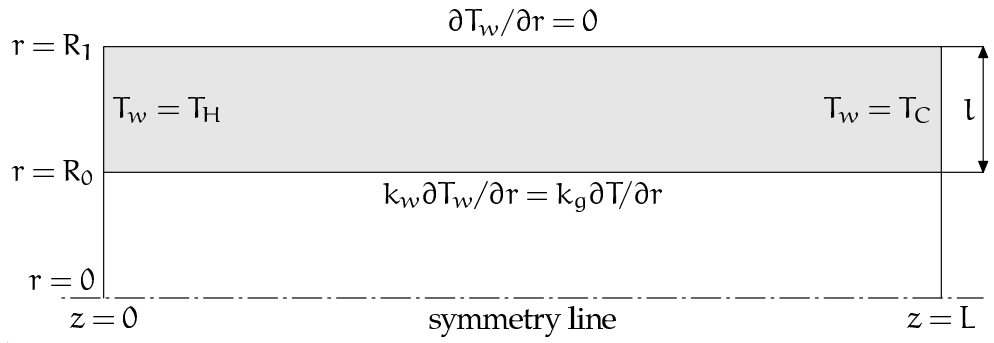

Figure 2.6: Wall domain with boundary conditions.

\subsubsection{Wall model}

The two-dimensional model allows us to study the local heat exchange between the gas and the wall. The wall of thickness $l$ is modelled using the same axisymmetric geometry as for the gas domain. We will solve the temperature equation for the wall simultaneously with the temperature equation for the gas. The domain under consideration together with applied boundary conditions is shown in Figure 2.6. During operation the pulse tube is placed in a vacuum chamber. It is therefore assumed that heat exchange with the surroundings and radiation losses are negligible. Thus the outer wall surface is adiabatic. At the interface between the wall and the gas continuity of temperature and heat flux is assumed. Energy conservation for the tube wall requires

$$
\rho_{w} c_{w} \frac{\partial \mathrm{T}_{w}}{\partial \mathrm{t}}=k_{w}\left(\frac{\partial^{2} \mathrm{~T}_{w}}{\partial \mathrm{r}^{2}}+\frac{1}{\mathrm{r}} \frac{\partial \mathrm{T}_{w}}{\partial \mathrm{r}}+\frac{\partial^{2} \mathrm{~T}_{w}}{\partial z^{2}}\right) .
$$

For the non-dimensionalisation of equation (2.110) the time and length scales are as for the flow: $t=\hat{t} / \omega, z=(\bar{u} / \omega) \hat{z}, r=(\bar{u} / \omega) \hat{r}$. In non-dimensional form equation (2.110) reads

$$
\frac{\partial \mathrm{T}_{w}}{\partial \mathrm{t}}=\alpha\left(\frac{\partial^{2} \mathrm{~T}_{w}}{\partial \mathrm{r}^{2}}+\frac{1}{\mathrm{r}} \frac{\partial \mathrm{T}_{w}}{\partial \mathrm{r}}+\frac{\partial^{2} \mathrm{~T}_{w}}{\partial z^{2}}\right) .
$$

The dimensionless thermal diffusivity coefficient $\alpha$, sometimes called Fourier number Fo, gives the ratio of the rate of heat conduction to the rate of thermal energy storage

$$
\alpha=\frac{k_{w} \omega}{\bar{\rho}_{w} c_{w} \bar{u}^{2}} .
$$

The wall temperature and the gas temperature are coupled through the dimensionless interface condition

$$
\frac{\partial T_{w}}{\partial r}=\beta \frac{\partial T}{\partial r} \text { with } \beta=\frac{k_{g}}{k_{w}} .
$$

For a typical single-inlet pulse tube, operating at $20 \mathrm{~Hz}, \alpha \sim 2.6 \times 10^{-4}$ and $\beta \sim 0.01$. 


\begin{tabular}{|c|c|c|c|c|}
\hline & $\mathrm{T}$ & $u$ & $v$ & $p$ \\
\hline hot end & $\left\{\begin{array}{l}T=T_{H}, \quad u \geq 0 \\
\frac{\partial T}{\partial z}=f(r, t), u<0\end{array}\right.$ & $\mathfrak{u}=\mathfrak{u}_{\mathrm{H}}$ & $v=0$ & \\
\hline cold end & $\left\{\begin{array}{l}T=T_{C}, \quad u \leq 0 \\
\frac{\partial T}{\partial z}=g(r, t), u>0\end{array}\right.$ & & $v=0$ & $p=0$ \\
\hline wall & $\left\{\begin{array}{l}k_{g} \partial \mathrm{T} / \partial \mathrm{r}=\mathrm{k}_{w} \partial \mathrm{T}_{w} / \partial \mathrm{r} \\
\mathrm{T}=\mathrm{T}_{w}\end{array}\right.$ & $\mathrm{u}=0$ & $v=0$ & \\
\hline symmetry & $\partial \mathrm{T} / \partial \mathrm{r}=0$ & $\partial u / \partial r=0$ & $v=0$ & $\partial p / \partial r=0$ \\
\hline
\end{tabular}

Table 2.1: Boundary conditions for the two-dimensional model.

\subsubsection{Boundary and initial conditions}

The mathematical model is not complete without specifying the boundary and initial conditions. In this section the boundary and initial conditions for the equations (2.103), (2.104) and (2.109) are given.

The choice of boundary conditions for pressure and velocities is an art in itself, see [23], [55]. It is of great importance that we supply physically realistic, well-posed boundary conditions. For the flow equations (2.103) - (2.105) we use the following configuration: wall, one boundary with given velocities, one boundary with fixed pressure and a radial symmetry boundary. So we prescribe the velocities at the hot end and the first-order pressure at the cold end. The first-order pressure is a somewhat mysterious quantity. It is not a thermodynamic variable. It is in one sense a mathematical artifact - a Lagrange multiplier that ensures that the velocity divergence constraint is satisfied. Yet its gradient is a relevant physical quantity - a force per unit volume. We set the first-order pressure at the cold end equal to zero. It means that at this boundary the total pressure is equal to thermodynamic pressure. At the symmetry line we impose zero radial derivatives for all variables except for the radial velocity, which itself must be zero at the centre line.

The boundary conditions for the temperature equation (2.109) at the hot and cold ends are the same as in the one-dimensional model, equations (2.54), (2.55), except 
for possible r-dependence. They read

$$
\begin{gathered}
\left\{\begin{array}{l}
T(0, r, t)=T_{H} \text { if } u(0, r, t) \geq 0 \\
\frac{\partial T}{\partial z}(0, r, t)=f(r, t) \text { if } u(0, r, t)<0
\end{array}\right. \\
f(r, t)=\left(s_{2}(t) T(0, r, t)-\frac{\partial T}{\partial t}(0, r, t)\right) / u(0, r, t), \\
\left\{\begin{array}{l}
T(L, r, t)=T_{C} \text { if } u(L, r, t) \leq 0 \\
\frac{\partial T}{\partial z}(L, r, t)=g(r, t) \text { if } u(L, t)>0
\end{array}\right. \\
g(r, t)=\left(s_{2}(t) T(L, r, t)-\frac{\partial T}{\partial t}(L, r, t)\right) / u(L, r, t) .
\end{gathered}
$$

All physical boundary conditions required by the continuous problem are summarised in Table 2.1. Initial conditions have to be described for all five variables. One possibility is the no-flow steady state given by

$$
\mathrm{T}=\mathrm{T}_{\mathrm{H}}-\left(\mathrm{T}_{\mathrm{H}}-\mathrm{T}_{\mathrm{C}}\right) z / \mathrm{L}, \mathrm{u}=0, v=0, p=0, \mathrm{~T}_{w}=\mathrm{T} .
$$




\section{CHAPTER 3}

\section{Numerical solution methods for 1D equations}

In this chapter the numerical methods for the solution of the resulting system of equations in one-dimensional form will be presented. The governing equations are discretised using finite difference techniques. The temperature equation is a convectiondiffusion equation. We use an implicit scheme for the diffusive term and an explicit scheme with flux limiter for the convective term. The velocity in the onedimensional case can be found from the constraint equation. Although there is no need to compute the first-order pressure in the one-dimensional case, we investigate different pressure-correction algorithms for the momentum and constraint equations to find the optimal scheme for our flow problem. It will be used in solving the twodimensional system. Finally, we explain how more accurate solutions can be obtained by grid refinement. Instead of applying non-uniform locally refined grids, we use several uniform grids with different mesh sizes. This refinement strategy known as local uniform grid refinement (LUGR) is examined.

\subsection{Velocity and temperature computation}

The starting point of any numerical method is the mathematical model, here a set of partial differential equations, boundary and initial conditions. In chapter 2 , section 2.3, we derived the final system of equations for the one-dimensional case

$$
\begin{gathered}
\frac{\partial u}{\partial x}=\epsilon \frac{\partial^{2} T}{\partial x^{2}}+s_{1}(t), \\
\frac{\partial T}{\partial t}=\epsilon T \frac{\partial^{2} T}{\partial x^{2}}-u \frac{\partial T}{\partial x}+s_{2}(t) T \\
s_{1}(t)=-\frac{1}{(\mathcal{A}+P(t)) \gamma} \frac{d P(t)}{d t}
\end{gathered}
$$




$$
\begin{aligned}
\mathrm{s}_{2}(\mathrm{t}) & =\frac{1}{(\mathcal{A}+\mathrm{P}(\mathrm{t}))} \frac{\gamma-1}{\gamma} \frac{\mathrm{dP}(\mathrm{t})}{\mathrm{dt}}, \\
\epsilon(\mathrm{t}) & =\frac{1}{\mathcal{B}(\mathcal{A}+\mathrm{P}(\mathrm{t}))} \frac{1}{\mathrm{Pe}} \ll 1,
\end{aligned}
$$

together with the boundary conditions

$$
\begin{gathered}
u(0, t)=u_{H}(t)=-\mathcal{C}\left(P(t)+\mathcal{A}-\mathcal{E} p_{b}(t)\right), \\
\left\{\begin{array}{l}
T(0, t)=T_{H} \text { if } u(0, t) \geq 0 \\
u(0, t) \frac{\partial T}{\partial x}(0, t)=s_{2}(t) T(0, t)-\frac{\partial T}{\partial t}(0, t) \text { if } u(0, t)<0
\end{array}\right. \\
\left\{\begin{array}{l}
T(L, t)=T_{C} \text { if } u(L, t) \leq 0 \\
u(L, t) \frac{\partial T}{\partial x}(L, t)=s_{2}(t) T(L, t)-\frac{\partial T}{\partial t}(L, t) \text { if } u(L, t)>0
\end{array}\right.
\end{gathered}
$$

and initial conditions

$$
\begin{gathered}
T(x, 0)=T_{H}-\left(T_{H}-T_{C}\right) \frac{x}{L}, \\
u(x, 0)=s_{1}(0) x+u_{H}(0) .
\end{gathered}
$$

For the numerical solution of the equations (3.1), (3.2) one has to choose a suitable discretisation method, i.e. a method of approximating the differential equations by a system of algebraic equations for the variables at some set of discrete points in space and time. There are many approaches, but the most important are: the finite difference method (FDM), the finite volume method (FVM) and the finite element method (FEM). The areas of application for each method follow logically from its advantages and disadvantages.

FDM is the oldest method for the numerical solution of partial differential equations, believed to have been introduced by Euler in the 18th century. The starting point is an equation in differential form and a grid covering the domain. At each grid point, the differential equation is approximated by replacing its partial derivatives by a combination of nodal values of the unknown function. The result is one algebraic equation per grid node. On structured grids, the FDM is very simple and effective. It is especially easy to obtain higher-order schemes on structured grids. But FDM is less efficient on non-structured grids. The restriction to simple geometries is a significant disadvantage. Another disadvantage of FDM is that special care has to be taken to check the conservation properties.

FVM uses the integral form of the conservation equations as its starting point. The solution domain is subdivided into a number of contiguous finite control volumes. FVM is called cell-centered if variable values are calculated in the centre of each control volume and vertex-centred if they are calculated at the vertices. Surface and volume integrals are approximated using suitable quadrature formulae. As a result, one obtains an algebraic equation for each control volume. The FVM can accommodate 
any grid type, so it is suitable for complex geometries. The FVM is relatively simple to understand and implement and all terms that need to be approximated have a clear physical meaning.

FEM is similar to FVM. The domain is divided into a set of discrete volumes or finite elements. For two- dimensional problems these usually are triangles or quadrilaterals. The main feature of FEM is that the differential equations arise from a variational formulation. The solution within each element is approximated by linear combinations of suitable basis functions such that continuity of the solution across element boundaries is guaranteed. An important advantage of FEM is the ability to deal with arbitrary geometries. The disadvantage, which is shared by any method that uses unstructured grids, is that the matrices representing the linearised equations are not as well structured as for regular grids, thus making it more difficult to find efficient solution methods.

When choosing a proper solution method for our problem, we have to keep in mind the geometry of the domain and the specific nature of the governing equations. Most pulse tubes have a simple cylindrical geometry, which makes the use of either FDM or FVM attractive. The conservation equations herein are written in differential form with primitive (non-conservative) variables, so that the FDM is the logical choice.

In the FDM, we introduce a uniform spatial grid $\left\{x_{j}=j h, j=0, \ldots, N_{x}\right\}$ with grid size $h=L / N_{x}$ and time levels $\left\{t^{n}=n \tau^{n}, n=0, \ldots, N_{t}\right\}$ with variable time step $\tau^{n}$. Velocity and temperature at grid point $\left(x_{j}, t^{n}\right)$ are denoted as $u_{j}^{n}$ and $T_{j}^{n}$ respectively. We decouple the velocity equation from the temperature equation by using velocity values from the previous time level.

For the one-dimensional case we find the velocity from the velocity divergence constraint, equation (3.1). The following formulae for the velocity computation have been used

$$
\begin{array}{cr}
u_{j}^{n}=u_{j-1}^{n}+\frac{\epsilon^{n}}{h}\left(T_{j-1}^{n}-2 T_{j}^{n}+T_{j+1}^{n}\right)+h s_{1}\left(t^{n}\right) \quad j=1, \ldots, N_{x}-1, \\
u_{N_{x}}^{n}=u_{N_{x}-1}^{n}+\frac{\epsilon^{n}}{h}\left(T_{N_{x}}^{n}-2 T_{N_{x}-1}^{n}+T_{N_{x}-2}^{n}\right)+h s_{1}\left(t^{n}\right) & j=N_{x}, \\
u_{0}^{n}=u_{H}^{n} & j=0,
\end{array}
$$

for every time level $n=1,2,3, \ldots, N_{t}$ with $u_{H}(t)$ given.

To solve the convection-dominated temperature equation we have chosen the following approach:

- Convection. Sharp resolution of jumps without excessive smearing requires explicit time discretisation and a high-resolution scheme.

- Diffusion. Explicit schemes lead to stability conditions of the type $\tau=\mathrm{O}(\mathrm{h})$ for the convection term and $\tau=\mathrm{O}\left(\mathrm{h}^{2}\right)$ for the diffusion term. The last condition is too severe. One of the possibilities to avoid this restriction is to discretise the diffusion term implicitly. 
The approximation of the convection term depends on the velocity sign, indicating the flow direction.

If $u_{j}^{n}>0$, we use the following scheme for the temperature equation

$$
\begin{gathered}
T_{j}^{n+1}-\epsilon^{n} \tau^{n} T_{j}^{n} \frac{T_{j-1}^{n+1}-2 T_{j}^{n+1}+T_{j+1}^{n+1}}{h^{2}}-\tau^{n} s_{2}\left(t^{n+1}\right) T_{j}^{n+1}= \\
T_{j}^{n}-c_{j}^{n}\left(1+\frac{1}{2}\left(1-c_{j}^{n}\right)\left(\frac{\Psi_{j+\frac{1}{2}}^{n}}{\theta_{j+\frac{1}{2}}^{n}}-\Psi_{j-\frac{1}{2}}^{n}\right)\right)\left(T_{j}^{n}-T_{j-1}^{n}\right) \\
j=2, \ldots, N_{x}-2, \quad n=0, \ldots, N_{t}-1 .
\end{gathered}
$$

If $u_{j}^{n}<0$, then

$$
\begin{gathered}
T_{j}^{n+1}-\epsilon^{n} \tau^{n} T_{j}^{n} \frac{T_{j-1}^{n+1}-2 T_{j}^{n+1}+T_{j+1}^{n+1}}{h^{2}}-\tau^{n} s_{2}\left(t^{n+1}\right) T_{j}^{n+1}= \\
T_{j}^{n}-c_{j}^{n}\left(1-\frac{1}{2}\left(1+c_{j}^{n}\right)\left(\Psi_{j+\frac{1}{2}}^{n}-\frac{\Psi_{j-\frac{1}{2}}^{n}}{\theta_{j-\frac{1}{2}}^{n}}\right)\right)\left(T_{j+1}^{n}-T_{j}^{n}\right) \\
j=2, \ldots, N_{x}-2, \quad n=0, \ldots, N_{t}-1,
\end{gathered}
$$

where $c_{j}^{n}$ is the Courant number, $c_{j}^{n}:=\tau^{n} u_{j}^{n} / h$. The ratio $\theta_{j+\frac{1}{2}}^{n}$ is defined by

$$
\theta_{j+\frac{1}{2}}^{n}:=\left\{\begin{array}{l}
\frac{T_{j}^{n}-T_{j-1}^{n}}{T_{j+1}^{n}-T_{j^{\prime}}^{n}} \text { if } u_{i}^{n}>0 \\
\frac{T_{j+2}^{n}-T_{j+1}^{n}}{T_{j+1}^{n}-T_{j^{\prime}}^{n}} \text { if } u_{i}^{n}<0
\end{array} \quad, \theta_{j-\frac{1}{2}}^{n}:=\left\{\begin{array}{l}
\frac{T_{j-1}^{n}-T_{j-2}^{n}}{T_{j}^{n}-T_{j^{\prime}-1}^{n}} \text { if } u_{i}^{n}>0 \\
\frac{T_{j+1}^{n}-T_{j}^{n}}{T_{j}^{n}-T_{j-1}^{n}} \text { if } u_{i}^{n}<0
\end{array},\right.\right.
$$

and $\Psi_{j \pm \frac{1}{2}}^{n}:=\Psi\left(\theta_{j \pm \frac{1}{2}}^{n}\right)$ is the flux limiter. Possible choices are the smooth Van Leer limiter

$$
\Psi(\theta)=\frac{\theta+|\theta|}{1+|\theta|}
$$

or the minmod limiter [26, p. 542] defined by

$$
\Psi(\theta)=\max (0, \min (\theta, 1)) .
$$

For $\theta \leq 0$, the limiter function $\Psi(\theta)=0$. This means that in the vicinity of steep gradients, where $\theta_{j+\frac{1}{2}}^{n}<0, \theta_{j-\frac{1}{2}}^{n}<0$, the high-resolution schemes (3.12) and (3.13) reduce to upwind schemes. Computing $\mathrm{T}_{j}^{n+1}$ with formulae (3.12) and (3.13) requires values from the neighbouring grid points $j-2, j-1, j, j+1, j+2$. For $j=1$ and $j=N_{x}-1$ the standard upwind scheme is used $(\Psi(\theta)=0$ ). If the CFL (CourantFriedrichs-Lewy) stability condition $\left|c_{j}^{\mathfrak{n}}\right| \leq 1$ or, equivalently,

$$
\tau^{n} \leq h / \max _{j}\left|u_{j}^{n}\right|
$$


is satisfied, both schemes (3.12) and (3.13) are second-order accurate in space (away from discontinuities) and first-order accurate in time, see [49]. The discretised boundary conditions (3.7), (3.8) are

$$
\begin{array}{r}
T_{0}^{n+1}+\tau^{n} u_{0}^{n} \frac{T_{1}^{n+1}-T_{0}^{n+1}}{h}-\tau^{n} s_{2}\left(t^{n+1}\right) T_{0}^{n+1}=T_{0}^{n}, \\
T_{N_{x}}^{n+1}+\tau^{n} u_{N_{x}}^{n} \frac{T_{N_{x}}^{n+1}-T_{N_{x}-1}^{n+1}}{h}-\tau^{n} s_{2}\left(t^{n+1}\right) T_{N_{x}}^{n+1}=T_{N_{x}}^{n} .
\end{array}
$$

The numerical algorithm is summarized as follows. Assume that the initial temperature $T_{j}^{0}$ and velocity $u_{j}^{0}$ are given. At time step $n+1$ :

1. Estimate $\max _{j}\left|u_{j}^{n}\right|$ at the previous time level and define $\tau^{n}$ according to the CFL condition (3.17).

2. Compute the temperature $T_{j}^{n+1}$ from (3.12) if $u_{j}^{n}>0$ or from (3.13) if $u_{j}^{n}<0$, using $T_{j}^{n}$ and $u_{j}^{n}$.

3. Compute the velocity $u_{j}^{n+1}$ via (3.11), using $T_{j}^{n+1}$.

\subsection{Pressure correction algorithms for the 1D case}

In anticipation to the two-dimensional case, let us consider the system of leadingorder equations but now in combination with the first-order momentum equation. It has the leading-order pressure $\mathrm{P}(\mathrm{t})$ as a given function of time and the first-order pressure $p$ as unknown.

$$
\begin{gathered}
\rho\left(\frac{\partial u}{\partial t}+u \frac{\partial u}{\partial x}\right)=-\frac{\partial p}{\partial x}+\frac{4}{3 \operatorname{Re}} \frac{\partial^{2} u}{\partial x^{2}} \\
\frac{\partial u}{\partial x}=\epsilon \frac{\partial^{2} T}{\partial x^{2}}+s_{1}(t) \\
\frac{\partial T}{\partial t}=\epsilon T \frac{\partial^{2} T}{\partial x^{2}}-u \frac{\partial T}{\partial x}+s_{2}(t) T \\
(\mathcal{A}+P(t)) \mathcal{B}=\rho T .
\end{gathered}
$$

In the one-dimensional case there is no need to compute the first-order pressure, because the constraint equation (3.20) gives us a completely defined velocity field. But for higher dimensions this is not the case.

To deal with the problem of pressure-velocity coupling, we employ the pressure correction method. Introduced in 1967 in [10], pressure correction schemes have been the topic of research for more than thirty years. One of the most well known and widely used methods is SIMPLE (Semi-Implicit Method for Pressure-Linked Equations), proposed in [54]. This algorithm was designed for stationary incompressible 
flow problems. Many variations and improvements have been proposed, such as SIMPLER [53], SIMPLEC [11], SIMPLEN [71]. The method can be also extended to compressible flow computations [16]. In [33] the PISO (Pressure-Implicit with Splitting of Operators) method was introduced, which especially aimed at solving unsteady flow problems. The SIMPLE and PISO schemes have strong similarities, both in terms of algorithmic formulation and general methodology. All pressure correction methods start from computing a guess for the velocity field in the predictor step, using initial approximations of velocity and pressure. The predictor value of the velocity field typically does not satisfy the velocity constraint equation and is corrected in the corrector step. Since this correction affects the pressure field, a related pressure correction is defined, obtained by requiring that the corrected velocity satisfies the constraint. This leads to a Poisson equation for the pressure correction. A whole variety of methods can be formulated by varying the number of prediction and correction steps used, see [5].

The main questions to be answered in this section are:

- What is a suitable pressure correction algorithm for the low-Mach-number compressible unsteady flows? With some imagination many methods can be invented to determine the solution at the new time level. When making our choice we keep in mind requirements such as simplicity, stability and accuracy.

- How many corrector steps are needed?

- What are the proper boundary conditions for velocity and first-order pressure? As was shown in Section 2.3.3, in the one-dimensional problem only one physical boundary condition for the velocity is needed to define the velocity field. Sometimes knowing which physical boundary conditions to impose is not enough to solve the problem numerically, extra information must be supplied in the form of numerical boundary conditions. These appear to be needed by the numerical method whilst not being explicitly given by the physics of the problem. The numerical ("soft") boundary conditions depend on grid, discretisation technique and solution method. The pressure correction algorithms require two boundary conditions for velocity and two boundary conditions for pressure.

\subsubsection{A model problem}

To test different pressure correction algorithms we construct a model problem in which the pressure equation can be easily elaborated. For this purpose an isothermal inviscid flow in an open tube subject to sinusoidal pressure variation is considered.

The domain of interest is a one-dimensional region $0<x<L$, where $L$ is the length of the tube. The unknowns are velocity $u(x, t)$ and hydrodynamic pressure $p(x, t)$. The system of equations is

$$
\begin{aligned}
& \frac{\partial \rho}{\partial t}+u \frac{\partial \rho}{\partial x}+\rho \frac{\partial u}{\partial x}=0 \\
& \frac{\partial u}{\partial t}+u \frac{\partial u}{\partial x}=-\frac{1}{\rho} \frac{\partial p}{\partial x}
\end{aligned}
$$


with boundary conditions

$$
u(0, t)=u_{H}(t)=-\mathcal{C P}(t),
$$

and density and driving pressure given by

$$
(\mathcal{A}+\mathrm{P}(\mathrm{t})) \mathcal{B}=\rho \text { with } \mathrm{P}(\mathrm{t})=\sin (\mathrm{t}) .
$$

Substitution of the density, given by (3.26), in the continuity equation (3.23) gives a velocity divergence constraint

$$
\frac{\partial u}{\partial x}=s(t) \text { with } s(t)=-\frac{1}{(\mathcal{A}+P(t))} \frac{d P}{d t} .
$$

Combining the equations (3.24) and (3.27), we derive

$$
-\frac{1}{\rho} \frac{\partial p}{\partial x}=\left(s^{2}(t)+s^{\prime}(t)\right) x+\left(u_{H}^{\prime}(t)+s(t) u_{H}(t)\right) .
$$

Differentiation of equation (3.28) gives a Poisson equation for the pressure, which can be written as

$$
\frac{\partial^{2} p}{\partial x^{2}}=-\rho(t)\left(s^{2}(t)+s^{\prime}(t)\right) .
$$

The Neumann boundary conditions for the pressure follow directly from (3.28) :

$$
\begin{gathered}
x=0: \quad \frac{\partial p}{\partial x}=-\rho(t)\left(u_{H}^{\prime}(t)+s(t) u_{H}(t)\right), \\
x=L: \quad \frac{\partial p}{\partial x}=-\rho(t) L\left(s^{\prime}(t)+s^{2}(t)\right)-\rho(t)\left(u_{H}^{\prime}(t)+s(t) u_{H}(t)\right) .
\end{gathered}
$$

Integration of equation (3.28) gives

$$
p(t, x)=-\rho(t)\left[\frac{1}{2}\left(s^{2}(t)+s^{\prime}(t)\right) x^{2}+\left(\mathfrak{u}_{H}^{\prime}(t)+s(t) u_{H}(t)\right) x\right]+F(t) .
$$

The system of equations (3.23) - (3.24) defines the pressure up to the unknown function $F(t)$. To have a unique pressure we fix it at one point - at the boundary $x=L$. If we set $p(t, L)=0$, the term $F(t)$ can be found from (3.32) as

$$
F(t)=\rho(t)\left[\frac{1}{2}\left(s^{2}(t)+s^{\prime}(t)\right) L^{2}+\left(u_{H}^{\prime}(t)+s(t) u_{H}(t)\right) L\right] .
$$

\section{Formulation of algorithms}

All proposed algorithms will use the momentum equation (3.24) and the velocity divergence constraint equation (3.27) for the determination of the pressure and the velocity field. Usually the continuity and momentum equation are used to construct pressure correction schemes for compressible flows. The explanation is that the pressure correction schemes have originally been developed for incompressible 
flows, for which the continuity equation and the constraint equation are identical. For compressible flows, the constraint equation is a combination of the continuity and the energy equations. Pressure correction algorithms constructed with the continuity equation are different from those constructed with constraint equations. In [27], on the basis of stability and accuracy considerations, the continuity correction methods were shown to be inappropriate for low-Mach-number compressible flows.

The system of equations for velocity and pressure can be written in terms of grad and div operators. This enables a convenient extension to higher dimensions.

$$
\begin{gathered}
\frac{\partial u}{\partial t}=u \frac{\partial u}{\partial x}-\frac{1}{\rho} \operatorname{grad} p \\
\operatorname{div} u=s(t) .
\end{gathered}
$$

Let us construct algorithms for computing $p^{n+1}, u^{n+1}$, assuming that $\rho^{n+1}$ is already known. We use a collocated grid where all unknowns are computed at the same grid points. The alternative is a staggered grid where grid points for different unknowns are staggered with respect to each other. These grids were introduced in the sixties for incompressible flow computation to overcome difficulties with pressure-velocity coupling and the occurrence of oscillations in the pressure (checkerboard instability). Despite the strong coupling between velocities and pressure, staggered mesh schemes have drawbacks. When used with non-uniform grids, the discretisation is more difficult. This adds considerably to the programming effort and to the computational time, especially when adaptive or multigrid mesh approaches are used.

\section{ALGORITHM 1}

prediction step:

First a 'predictor' velocity field $u^{*}$ is determined by solving the following implicit equation

$$
\frac{u_{i}^{*}-u_{i}^{n}}{\tau}=H\left(u_{i}^{*}\right)-\frac{1}{\rho_{i}^{n+1}} \operatorname{grad}\left(p_{i}^{n}\right) .
$$

The operator $\mathrm{H}\left(\mathrm{u}_{i}^{*}\right)$ stands for the finite-difference representation of the convective

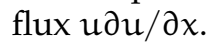

correction step:

$$
\frac{u_{i}^{n+1}-u_{i}^{n}}{\tau}=H\left(u_{i}^{*}\right)-\frac{1}{\rho_{i}^{n+1}} \operatorname{grad}\left(p_{i}^{n+1}\right)
$$

In this step the discretised momentum equation is written for $u_{i}^{n+1}$ in an explicit way. Equation (3.37) uses $\mathrm{H}\left(\mathrm{u}_{i}^{*}\right)$ instead of $\mathrm{H}\left(\mathrm{u}_{\mathrm{i}}^{\mathrm{n}+1}\right)$, assuming that the difference is negligibly small. Subtraction of (3.36) from (3.37) gives the equation for the velocity correction

$$
u_{i}^{n+1}=u_{i}^{*}-\frac{\tau}{\rho_{i}^{n+1}} \operatorname{grad}\left(p_{i}^{n+1}-p_{i}^{n}\right)
$$


pressure correction equation:

We substitute (3.38) in the velocity divergence constraint (3.35) so that

$$
\operatorname{div}\left(u_{i}^{n+1}\right)=s\left(t^{n+1}\right)
$$

and derive the Poisson equation for the pressure correction

$$
-\operatorname{div} \frac{\tau}{\rho_{i}^{n+1}} \operatorname{grad}\left(p_{i}^{n+1}-p_{i}^{n}\right)=s\left(t^{n+1}\right)-\operatorname{div}\left(u_{i}^{*}\right) .
$$

ALGORITHM 2

prediction step:

$$
\frac{u_{i}^{*}-u_{i}^{n}}{\tau}=H\left(u_{i}^{*}\right)-\frac{1}{\rho_{i}^{n+1}} \operatorname{grad}\left(p_{i}^{n}\right) .
$$

Now we decompose the operator $\mathrm{H}\left(u_{i}^{*}\right)$ into two parts

$$
H\left(u_{i}^{*}\right)=H^{\prime}\left(u_{i}^{*}\right)+A_{i}^{n} u_{i}^{*},
$$

where $A_{i}^{n}$ represents the coefficient of the central (i.e. diagonal) element. For most practical spatial difference schemes $A_{i}^{n}$ is a finite negative number. Equation (3.36) then becomes

$$
\left(1-\tau A_{i}^{n}\right) u_{i}^{*}=u_{i}^{n}+\tau H^{\prime}\left(u_{i}^{*}\right)-\frac{\tau}{\rho_{i}^{n+1}} \operatorname{grad}\left(p_{i}^{n}\right)
$$

correction step:

In this scheme the corrector step is partly implicit. The central element is moved to the left-hand side and treated implicitly. The rest of the operator $\mathrm{H}$ is retained on the right-hand side where it is treated explicitly. Equation (3.37) becomes

$$
\left(1-\tau A_{i}^{n}\right) u_{i}^{n+1}=u_{i}^{n}+\tau H^{\prime}\left(u_{i}^{*}\right)-\frac{\tau}{\rho_{i}^{n+1}} \operatorname{grad}\left(p_{i}^{n+1}\right) .
$$

Equation (3.43) uses $\mathrm{H}^{\prime}\left(\mathrm{u}_{i}^{*}\right)$ instead of $\mathrm{H}^{\prime}\left(\mathrm{u}_{i}^{\mathrm{n}+1}\right)$ only in the neighbouring points. Subtracting (3.42) from (3.43), the formula for the velocity correction is

$$
\begin{gathered}
u_{i}^{n+1}=u_{i}^{*}-\frac{\tau}{\rho_{i}^{n+1}} \frac{1}{B_{i}^{n}} \operatorname{grad}\left(p_{i}^{n+1}-p_{i}^{n}\right), \\
B_{i}^{n}=1-\tau A_{i}^{n} .
\end{gathered}
$$

pressure correction equation:

$$
-\operatorname{div} \frac{\tau}{\rho_{i}^{n+1}} \frac{1}{B_{i}^{n}} \operatorname{grad}\left(p_{i}^{n+1}-p_{i}^{n}\right)=s\left(t^{n+1}\right)-\operatorname{div}\left(u_{i}^{*}\right) .
$$

\section{ALGORITHM 3}


This algorithm closely resembles the Issa algorithm from [33], where the correction step is performed two times at every time level. The deviation is in the constraint equation, which was not used by Issa. The first three steps from ALGORITHM 1 are repeated with $p^{n+1}$ replaced by $p^{*}$ and $u^{n+1}$ replaced by $u^{* *}$.

prediction step:

$$
\frac{u_{i}^{*}-u_{i}^{n}}{\tau}=H\left(u_{i}^{*}\right)-\frac{1}{\rho_{i}^{n+1}} \operatorname{grad}\left(p_{i}^{n}\right) .
$$

first correction step:

$$
u_{i}^{* *}=u_{i}^{n}+\tau H\left(u_{i}^{*}\right)-\frac{\tau}{\rho_{i}^{n+1}} \operatorname{grad}\left(p_{i}^{*}\right) .
$$

Subtracting (3.46) from (3.47) yields a formula for the velocity correction,

$$
u_{i}^{* *}=u_{i}^{*}-\frac{\tau}{\rho_{i}^{n+1}} \operatorname{grad}\left(p_{i}^{*}-p_{i}^{n}\right) .
$$

pressure correction equation:

$$
\begin{gathered}
\operatorname{div}\left(u_{i}^{* *}\right)=s\left(t^{n+1}\right), \\
-\operatorname{div} \frac{\tau}{\rho_{i}^{n+1}} \operatorname{grad}\left(p_{i}^{*}-p_{i}^{n}\right)=s\left(t^{n+1}\right)-\operatorname{div}\left(u_{i}^{*}\right) .
\end{gathered}
$$

second correction step:

$$
\begin{aligned}
& \frac{u_{i}^{n+1}-u_{i}^{n}}{\tau}=H\left(u_{i}^{* *}\right)-\frac{1}{\rho_{i}^{n+1}} \operatorname{grad}\left(p_{i}^{n+1}\right), \\
& u_{i}^{n+1}=u_{i}^{n}+\tau H\left(u_{i}^{* *}\right)-\frac{\tau}{\rho_{i}^{n+1}} \operatorname{grad}\left(p_{i}^{n+1}\right) .
\end{aligned}
$$

Subtracting (3.47) from (3.52), we find the velocity correction,

$$
u_{i}^{n+1}=u_{i}^{* *}+\tau\left(H\left(u_{i}^{* *}\right)-H\left(u_{i}^{*}\right)\right)-\frac{\tau}{\rho_{i}^{n+1}} \operatorname{grad}\left(p_{i}^{n+1}-p_{i}^{*}\right) .
$$

second pressure correction equation:

$$
\begin{gathered}
\operatorname{div}\left(u_{i}^{n+1}\right)=s\left(t^{n+1}\right), \\
-\operatorname{div} \frac{\tau}{\rho_{i}^{n+1}} \operatorname{grad}\left(p_{i}^{n+1}-p_{i}^{*}\right)=s\left(t^{n+1}\right)-\operatorname{div}\left(u_{i}^{* *}\right)-\frac{\tau}{h} \operatorname{div}\left(H\left(u_{i}^{* *}\right)-H\left(u_{i}^{*}\right)\right) .
\end{gathered}
$$




\section{Spatial discretisation}

We use a collocated grid where the pressure and the other unknowns are defined at the same grid points. One-sided differences in the approximation of the pressure gradient and in the approximation of the divergence operator in the constraint equation prevents problems with checkerboard pressure at the expense of first-order accuracy. A proof of convergence of this method is given in [14].

\section{ALGORITHM 1}

prediction step:

$$
\frac{u_{i}^{*}-u_{i}^{n}}{\tau}=H\left(u_{i}^{*}\right)-\frac{1}{\rho_{i}^{n+1}} \frac{p_{i}^{n}-p_{i-1}^{n}}{h} .
$$

We linearise the convective term by using values from the previous time level.

$$
\begin{gathered}
H\left(u_{i}^{*}\right)=-u_{i+}^{n} \frac{u_{i}^{*}-u_{i-1}^{*}}{h}-u_{i-}^{n} \frac{u_{i+1}^{*}-u_{i}^{*}}{h}, \\
u_{i+}^{n}=\max \left(u_{i}^{n}, 0\right), \\
u_{i-}^{n}=\min \left(u_{i}^{n}, 0\right) .
\end{gathered}
$$

correction step:

$$
\frac{u_{i}^{n+1}-u_{i}^{n}}{\tau}=H\left(u_{i}^{*}\right)-\frac{1}{\rho_{i}^{n+1}} \frac{p_{i}^{n+1}-p_{i-1}^{n+1}}{h} .
$$

Subtracting (3.56) from (3.60) yields

$$
\begin{gathered}
u_{i}^{n+1}=u_{i}^{*}-\frac{\tau}{h} \frac{1}{\rho_{i}^{n+1}}\left(q_{i}-q_{i-1}\right), \\
q_{i}=p_{i}^{n+1}-p_{i}^{n} .
\end{gathered}
$$

pressure correction equation:

$$
\begin{gathered}
\frac{u_{i+1}^{n+1}-u_{i}^{n+1}}{h}=s\left(t^{n+1}\right) \\
-\frac{\tau}{h^{2}} \frac{1}{\rho_{i+1}^{n+1}}\left[q_{i+1}-q_{i}\right]+\frac{\tau}{h^{2}} \frac{1}{\rho_{i}^{n+1}}\left[q_{i}-q_{i-1}\right]=s\left(t^{n+1}\right)-\frac{u_{i+1}^{*}-u_{i}^{*}}{h} .
\end{gathered}
$$

The above procedure has been applied to all interior points $i=1, \ldots, N_{x}-1$. The boundary points are considered separately.

$i=0$ :

At the left boundary a Dirichlet boundary condition for velocity, see (3.25), is given at every time level

$$
u_{0}^{*}=u_{0}^{n+1}=-\mathcal{C P}\left(t^{n+1}\right) \text {. }
$$


The Neumann boundary condition for the pressure correction is derived from the constraint (3.63),

$$
\frac{u_{1}^{n+1}-u_{0}^{n+1}}{h}=s\left(t^{n+1}\right) \text { with } u_{0}^{n+1} \text { given. }
$$

Substituting (3.61), written at $i=1$, gives

$$
u_{1}^{n+1}=u_{1}^{*}-\frac{\tau}{h} \frac{1}{\rho_{1}^{n+1}}\left(q_{1}-q_{0}\right),
$$

which leads to the following boundary condition

$$
-\frac{\tau}{h^{2}} \frac{1}{\rho_{1}^{n+1}}\left[q_{1}-q_{0}\right]=s\left(t^{n+1}\right)-\frac{u_{1}^{*}-u_{0}^{n+1}}{h} .
$$

$i=N_{x}$ :

At the right boundary, the ' predictor' velocity $u_{N_{x}}^{*}$ is derived from the constraint equation

$$
u_{N_{x}}^{*}-u_{N_{x}-1}^{*}=h s\left(t^{n+1}\right) .
$$

The pressure correction has a Dirichlet boundary condition at this point

$$
\mathrm{q}_{\mathrm{N}_{\mathrm{x}}}=0 .
$$

\section{ALGORITHM 2}

The same discretisation procedure is applied to ALGORITHM 2.

predictor step:

The discretised equation (3.42) is

$$
\left(1-\tau A_{i}^{n}\right) u_{i}^{*}=u_{i}^{n}+\tau H^{\prime}\left(u_{i}^{*}\right)-\frac{\tau}{\rho_{i}^{n+1}}\left(p_{i}^{n}-p_{i-1}^{n}\right) .
$$

correction step:

The discretised equation (3.43) is

$$
\left(1-\tau A_{i}^{n}\right) u_{i}^{n+1}=u_{i}^{n}+\tau H^{\prime}\left(u_{i}^{*}\right)-\frac{\tau}{\rho_{i}^{n+1}} \frac{p_{i}^{n+1}-p_{i-1}^{n+1}}{h} .
$$

Subtracting (3.71) from (3.72), the formula for the velocity correction is now

$$
\begin{gathered}
\left(1-\tau A_{i}^{n}\right)\left(u_{i}^{n+1}-u_{i}^{*}\right)=-\frac{\tau}{h} \frac{1}{\rho_{i}^{n+1}}\left(p_{i}^{n+1}-p_{i-1}^{n+1}\right) . \\
u_{i}^{n+1}=u_{i}^{*}-\frac{\tau}{h} \frac{1}{\rho_{i}^{n+1}} \frac{1}{B_{i}^{n}}\left(q_{i}-q_{i-1}\right), \\
q_{i}=p_{i}^{n+1}-p_{i}^{n}, \quad B_{i}^{n}=1-\tau A_{i}^{n} .
\end{gathered}
$$


pressure correction equation:

$$
\begin{gathered}
\frac{u_{i+1}^{n+1}-u_{i}^{n+1}}{h}=s\left(t^{n+1}\right), \\
-\frac{\tau}{h^{2}} \frac{1}{\rho_{i+1}^{n+1}} \frac{1}{B_{i+1}^{n}}\left[q_{i+1}-q_{i}\right]+\frac{\tau}{h^{2}} \frac{1}{\rho_{i}^{n+1}} \frac{1}{B_{i}^{n}}\left[q_{i}-q_{i-1}\right]=s\left(t^{n+1}\right)-\frac{u_{i+1}^{*}-u_{i}^{*}}{h} .
\end{gathered}
$$

The boundary conditions are the same as in the previous algorithm.

\section{ALGORITHM 3}

prediction step:

$$
\frac{u_{i}^{*}-u_{i}^{n}}{\tau}=H\left(u_{i}^{*}\right)-\frac{1}{\rho_{i}^{n+1}} \frac{p_{i}^{n}-p_{i-1}^{n}}{h} .
$$

first correction step:

$$
\frac{u_{i}^{* *}-u_{i}^{n}}{\tau}=H\left(u_{i}^{*}\right)-\frac{1}{\rho_{i}^{n+1}} \frac{p_{i}^{*}-p_{i-1}^{*}}{h} .
$$

Subtracting (3.78) from (3.79), the formula for velocity correction is derived

$$
\begin{gathered}
u_{i}^{* *}=u_{i}^{*}-\frac{\tau}{h} \frac{1}{\rho_{i}^{n+1}}\left(q_{i}-q_{i-1}\right), \\
q_{i}=p_{i}^{*}-p_{i}^{n} .
\end{gathered}
$$

pressure correction equation:

$$
\begin{gathered}
\frac{u_{i+1}^{* *}-u_{i}^{* *}}{h}=s\left(t^{n+1}\right), \\
-\frac{\tau}{h^{2}} \frac{1}{\rho_{i+1}^{n+1}}\left[q_{i+1}-q_{i}\right]+\frac{\tau}{h^{2}} \frac{1}{\rho_{i}^{n+1}}\left[q_{i}-q_{i-1}\right]=s\left(t^{n+1}\right)-\frac{u_{i+1}^{*}-u_{i}^{*}}{h} .
\end{gathered}
$$

second correction step:

$$
\frac{u_{i}^{n+1}-u_{i}^{n}}{\tau}=H\left(u_{i}^{* *}\right)-\frac{1}{\rho_{i}^{n+1}} \frac{p_{i}^{n+1}-p_{i-1}^{n+1}}{h} .
$$

After subtracting equation (3.79) from (3.84) we get the following correction formula

$$
\begin{gathered}
u_{i}^{n+1}=u_{i}^{* *}+\tau\left(H\left(u_{i}^{* *}\right)-H\left(u_{i}^{*}\right)\right)-\frac{\tau}{h} \frac{1}{\rho_{i}^{n+1}}\left(q_{i}^{\prime}-q_{i-1}^{\prime}\right), \\
q_{i}^{\prime}=p_{i}^{n+1}-p_{i}^{*} .
\end{gathered}
$$

pressure correction equation:

$$
\frac{u_{i+1}^{n+1}-u_{i}^{n+1}}{h}=s\left(t^{n+1}\right),
$$




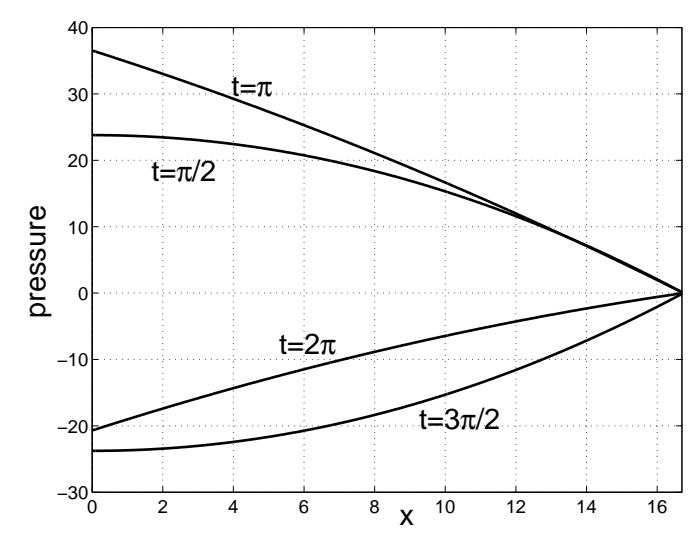

(a) pressure

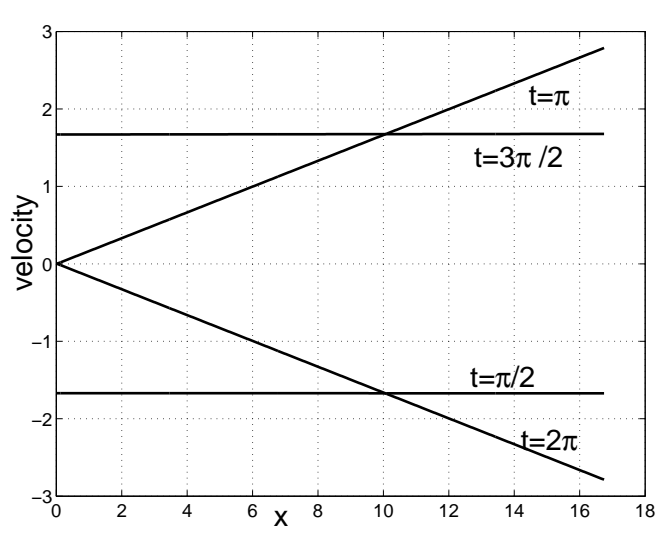

(b) velocity

Figure 3.1: Hydrodynamic pressure and velocity at times $t=\pi / 2, \pi, 3 \pi / 2$ and $2 \pi$.

$$
\begin{aligned}
& -\frac{\tau}{h^{2}} \frac{1}{\rho_{i+1}^{n+1}}\left[q_{i+1}^{\prime}-q_{i}^{\prime}\right]+\frac{\tau}{h^{2}} \frac{1}{\rho_{i}^{n+1}}\left[q_{i}^{\prime}-q_{i-1}^{\prime}\right]= \\
& \quad s\left(t^{n+1}\right)-\frac{u_{i+1}^{* *}-u_{i}^{* *}}{h}-\frac{\tau}{h}\left(H\left(u_{i+1}^{* *}\right)-H\left(u_{i+1}^{*}\right)\right)+\frac{\tau}{h}\left(H\left(u_{i}^{* *}\right)-H\left(u_{i}^{*}\right)\right) .
\end{aligned}
$$

\section{Results}

The performance of the described pressure correction schemes has been tested. The dimensionless parameter values are those of the pulse tube model, investigated in Chapter 5, namely

$$
\mathrm{L}=5.33 \pi, \mathcal{A}=6.0, \mathcal{B}=0.17, \mathcal{C}=1.67 .
$$

The numerical solution is computed from $t=0$ to $t=2 \pi$ on a grid with $N_{x}+1$ points and with $N_{t}$ time steps. The resulting systems of linear algebraic equations in the prediction step and in the pressure correction step have been solved using the iterative method BICGSTAB [59]. In combination with a preconditioner (incomplete LU-factorisation) this algorithm is fast and has a rather limited use of memory resources.

The hydrodynamic pressure and the velocity at times $t=\pi / 2, \pi, 3 \pi / 2$ and $2 \pi$ are given in Figure 3.1. At any time the velocity is a linear function of position. The accuracy is assessed from the maximum norm of the error in pressure $\left\|\epsilon_{N_{x}}\right\|_{\infty}=\|$ $p_{\text {exact }}-p_{N_{x}} \|_{\infty}$, where $p_{\text {exact }}$ is given by equation (3.32). Figure 3.2 indicates how the errors decay with $\tau=2 \pi / N_{t}$ for fixed $N_{x}=100$. It helps in estimating the optimal time step for each algorithm and also in comparing the accuracy of the different algorithms. From Figure 3.2 we may conclude that algorithm 2 allows larger time steps and is more accurate, especially for large $\tau$. The results of numerical tests with fixed $N_{x}$ and varying $N_{t}$ are also presented in Table 3.1. In Figure 3.3 the error 


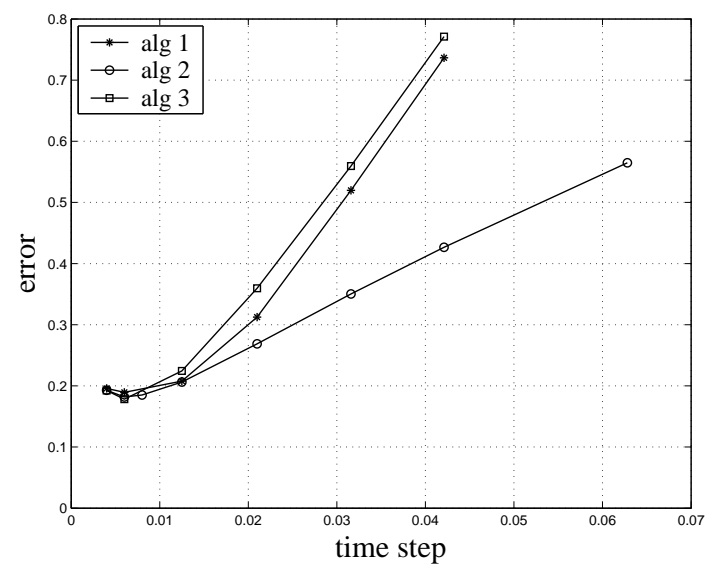

Figure 3.2: Error as function of time step $\tau$ for $N_{x}=100$ for three algorithms.

as function of time on successfully refined grids with $N_{x}=100(h=0.169), 200(h=$ $0.084)$ and $400(h=0.042)$ for all three algorithms is presented. Table 3.2 gives the maximum values of the error for refined grids.

The results of the simulation of the model problem show that

- Collocated grids and one-sided differences for the approximation of the grad and div operators produce a robust method which is first-order accurate in space and time.

- Algorithm 2 has better accuracy and allows larger time steps.

- Additional correction steps as in ALGORITHM 3 do not improve the solution in terms of maximum error in pressure.

- For the boundary conditions, we use a Dirichlet boundary condition for the velocity at the left boundary. The pressure correction has a Neumann condition

\begin{tabular}{cccc}
\hline$\tau$ & algorithm 1 & algorithm 2 & algorithm 3 \\
\hline \hline $\mathrm{N}_{\mathrm{t}}=200, \tau=0.0316$ & $5.19 \cdot 10^{-1}$ & $3.50 \cdot 10^{-1}$ & $5.59 \cdot 10^{-1}$ \\
$\mathrm{~N}_{\mathrm{t}}=300, \tau=0.0209$ & $3.13 \cdot 10^{-1}$ & $2.69 \cdot 10^{-1}$ & $3.60 \cdot 10^{-1}$ \\
$\mathrm{~N}_{\mathrm{t}}=500, \tau=0.0125$ & $2.08 \cdot 10^{-1}$ & $2.06 \cdot 10^{-1}$ & $2.24 \cdot 10^{-1}$ \\
\hline
\end{tabular}

Table 3.1: Maximum error in pressure $\left\|\epsilon_{N_{x}}\right\|_{\infty}, N_{x}=100(h=0.169)$ for the algorithms 1, 2, 3 with different $\tau$. 


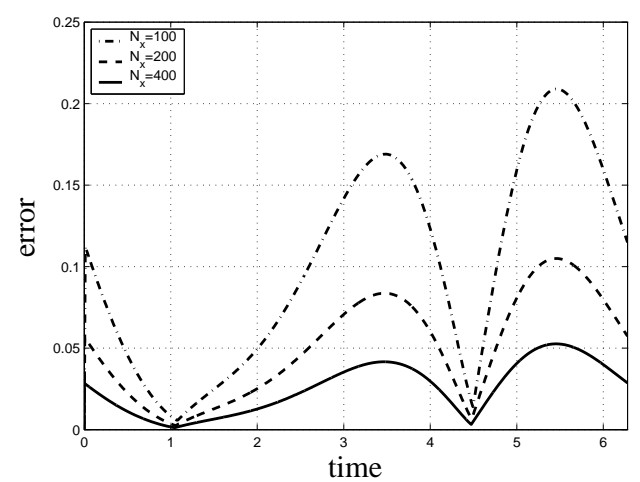

(a) algorithm 1

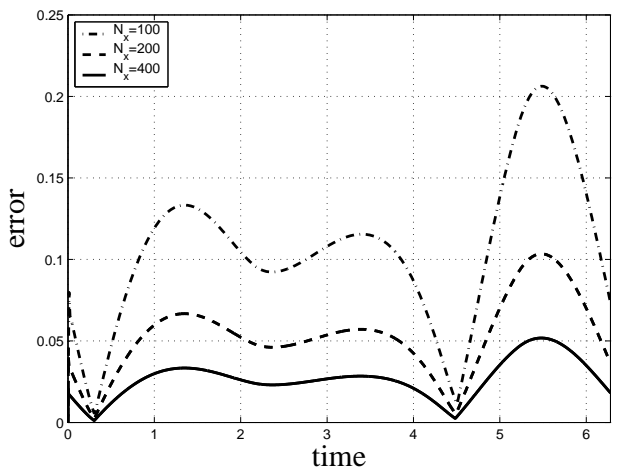

(b) algorithm 2

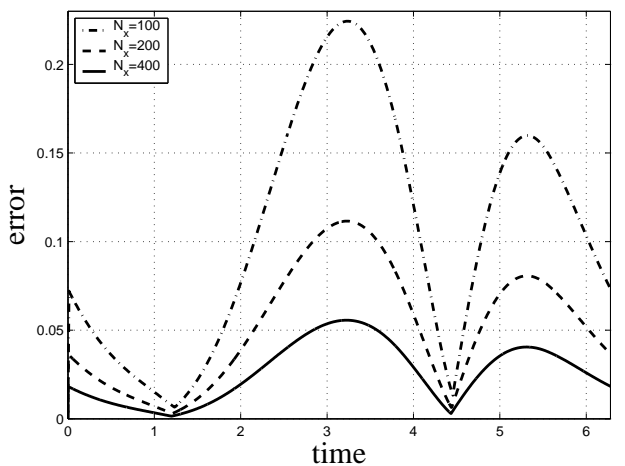

(c) algorithm 3

Figure 3.3: Error $\left\|\epsilon_{N_{x}}\right\|_{\infty}=\left\|p_{\text {exact }}-p_{N_{x}}\right\|_{\infty}$ as function of time. 


\begin{tabular}{cccc}
\hline$h, \tau$ & algorithm 1 & algorithm 2 & algorithm 3 \\
\hline \hline$h=0.169, \tau=0.0125$ & $2.08 \cdot 10^{-1}$ & $2.06 \cdot 10^{-1}$ & $2.24 \cdot 10^{-1}$ \\
$h=0.084, \tau=0.006$ & $1.05 \cdot 10^{-1}$ & $1.03 \cdot 10^{-1}$ & $1.11 \cdot 10^{-1}$ \\
$h=0.042, \tau=0.003$ & $5.26 \cdot 10^{-2}$ & $5.18 \cdot 10^{-2}$ & $5.56 \cdot 10^{-2}$ \\
\hline
\end{tabular}

Table 3.2: Maximum error in pressure $\left\|\epsilon_{N_{x}}\right\|_{\infty}$ for the algorithms 1, 2, 3 for different $h$.

at this boundary, which has been derived from the constraint equation. For the correct determination of the velocity field absolute values of the hydrodynamic pressure are not important: in the momentum equation only the pressure gradient appears. To have a unique solution for the pressure correction Poisson equation we must fix the pressure at one point. It seems reasonable to use a Dirichlet condition for the pressure at the right boundary, where the velocity has a Neumann condition. A fundamental discussion on boundary conditions for the pressure Poisson equation is presented in [23].

\subsubsection{Pulse tube flow}

In this section we apply the pressure correction ALGORITHM 2, which has shown the best performance in the model problem to the equations (3.19)-(3.22)

$$
\begin{gathered}
\rho\left(\frac{\partial u}{\partial t}+u \frac{\partial u}{\partial x}\right)=-\frac{\partial p}{\partial x}+\frac{4}{3 \operatorname{Re}} \frac{\partial^{2} u}{\partial x^{2}} \\
\frac{\partial u}{\partial x}=\epsilon \frac{\partial^{2} T}{\partial x^{2}}+s_{1}(t)
\end{gathered}
$$

with boundary condition (3.6). We assume that temperature and associated density at time level $n+1$ have already been computed from the equations (3.12) or (3.13) with time step $\tau^{n}$. Then velocity $u^{n+1}$ and pressure $p^{n+1}$ are found as follows.

$$
\begin{aligned}
& \text { prediction step: } \frac{u_{i}^{*}-u_{i}^{n}}{\tau^{n}}=H\left(u_{i}^{*}\right)-\frac{1}{\rho_{i}^{n+1}} \frac{p_{i}^{n}-p_{i-1}^{n}}{h}, \\
& H\left(u_{i}^{*}\right)=-u_{i+}^{n} \frac{u_{i}^{*}-u_{i-1}^{*}}{h}-u_{i-}^{n} \frac{u_{i+1}^{*}-u_{i}^{*}}{h}+\frac{4}{3 \operatorname{Re}} \frac{1}{\rho_{i}^{n+1}} \frac{u_{i+1}^{*}-2 u_{i}^{*}+u_{i-1}^{*}}{h^{2}} . \\
& H\left(u_{i}^{*}\right)=H^{\prime}\left(u_{i}^{*}\right)+A_{i}^{n} u_{i}^{*}, \\
& A_{i}^{n} u_{i}^{*}=-\frac{1}{h}\left(u_{i}^{n}-u_{i-}^{n}\right) u_{i}^{*}-\frac{4}{3 \operatorname{Re}} \frac{1}{\rho_{i}^{n+1}} \frac{2 u_{i}^{*}}{h^{2}}=-\frac{1}{h}\left|u_{i}^{n}\right| u_{i}^{*}-\frac{4}{3 \operatorname{Re}} \frac{1}{\rho_{i}^{n+1}} \frac{2 u_{i}^{*}}{h^{2}},
\end{aligned}
$$




$$
\begin{gathered}
A_{i}^{n}=-\frac{1}{h}\left|u_{i}^{n}\right|-\frac{4}{3 \operatorname{Re}} \frac{1}{\rho_{i}^{n+1}} \frac{2}{h^{2}}, \\
H^{\prime}\left(u_{i}^{*}\right)=u_{i}^{n} \frac{u_{i-1}^{*}}{h}-u_{i}^{n} \frac{u_{i+1}^{*}}{h}+\frac{4}{3 \operatorname{Re}} \frac{1}{\rho_{i}^{n+1}} \frac{u_{i+1}^{*}+u_{i-1}^{*}}{h^{2}} .
\end{gathered}
$$

Equation (3.92) is rewritten as

$$
\left(1-\tau^{n} A_{i}^{n}\right) u_{i}^{*}=u_{i}^{n}+\tau^{n} H^{\prime}\left(u_{i}^{*}\right)-\frac{\tau^{n}}{h} \frac{1}{\rho_{i}^{n+1}}\left(p_{i}^{n}-p_{i-1}^{n}\right) .
$$

correction step:

$$
\begin{gathered}
\frac{u_{i}^{n+1}-u_{i}^{n}}{\tau^{n}}=H\left(u_{i}^{*}\right)-\frac{1}{\rho_{i}^{n+1}} \frac{p_{i}^{n+1}-p_{i-1}^{n+1}}{h}, \\
\left(1-\tau^{n} A_{i}^{n}\right) u_{i}^{n+1}=u_{i}^{n}+\tau^{n} H^{\prime}\left(u_{i}^{*}\right)-\frac{\tau^{n}}{h} \frac{1}{\rho_{i}^{n+1}}\left(p_{i}^{n+1}-p_{i-1}^{n+1}\right) .
\end{gathered}
$$

Subtracting (3.98) from (3.100), the formula for the velocity correction is

$$
\begin{gathered}
\left(1-\tau^{n} A_{i}^{n}\right)\left(u_{i}^{n+1}-u_{i}^{*}\right)=-\frac{\tau^{n}}{h} \frac{1}{\rho_{i}^{n+1}}\left(p_{i}^{n+1}-p_{i-1}^{n+1}\right), \\
u_{i}^{n+1}=u_{i}^{*}-\frac{\tau^{n}}{h} \frac{1}{\rho_{i}^{n+1}} \frac{1}{B_{i}^{n}}\left(q_{i}-q_{i-1}\right), \quad B_{i}^{n}=1-\tau^{n} A_{i}^{n}, \\
q_{i}=p_{i}^{n+1}-p_{i}^{n} .
\end{gathered}
$$

pressure correction equation:

$$
\begin{gathered}
\frac{u_{i+1}^{n+1}-u_{i}^{n+1}}{h}=\epsilon^{n+1} \frac{T_{i-1}^{n+1}-2 T_{i}^{n+1}+T_{i+1}^{n+1}}{h^{2}}+s_{1}\left(t^{n+1}\right), \\
-\frac{\tau^{n}}{h^{2}} \frac{1}{\rho_{i+1}^{n+1}} \frac{1}{B_{i+1}^{n}}\left[q_{i+1}-q_{i}\right]+\frac{\tau^{n}}{h^{2}} \frac{1}{\rho_{i}^{n+1}} \frac{1}{B_{i}^{n}}\left[q_{i}-q_{i-1}\right]= \\
\epsilon^{n+1} \frac{T_{i-1}^{n+1}-2 T_{i}^{n+1}+T_{i+1}^{n+1}}{h^{2}}+s_{1}\left(t^{n+1}\right)-\frac{u_{i+1}^{*}-u_{i}^{*}}{h} .
\end{gathered}
$$

The above procedure is applied for the interior points $i=1, \ldots, N_{x}-1$. The boundary points are considered separately.

$i=0$ :

If the velocity has a Dirichlet boundary condition, then the pressure correction has a corresponding Neumann boundary condition, which is derived from the velocity constraint

$$
\frac{u_{1}^{n+1}-u_{0}^{n+1}}{h}=\epsilon^{n+1} \frac{T_{2}^{n+1}-2 T_{1}^{n+1}+T_{0}^{n+1}}{h^{2}}+s_{1}\left(t^{n+1}\right),
$$




$$
u_{0}^{n+1} \text { given. }
$$

Substituting equation (3.102), written for $i=1$,

$$
u_{1}^{n+1}=u_{1}^{*}-\frac{\tau^{n}}{h} \frac{1}{\rho_{1}^{n+1}} \frac{1}{B_{1}^{n}}\left(q_{1}-q_{0}\right)
$$

into equation (3.106) gives the boundary condition

$$
-\frac{\tau^{n}}{h^{2}} \frac{1}{\rho_{1}^{n+1}} \frac{1}{B_{1}^{n}}\left[q_{1}-q_{0}\right]=\epsilon^{n+1} \frac{T_{2}^{n+1}-2 T_{1}^{n+1}+T_{0}^{n+1}}{h^{2}}+s_{1}\left(t^{n+1}\right)-\frac{u_{1}^{*}-u_{0}^{n+1}}{h} .
$$

$i=N_{x}$ :

There is a Neumann boundary condition for the predictor velocity, derived from the constraint equation

$$
\frac{u_{N_{x}}^{*}-u_{N_{x}-1}^{*}}{h}=\epsilon^{n+1} \frac{T_{N_{x}}^{n+1}-2 T_{N_{x}-1}^{n+1}+T_{N_{x}-2}^{n+1}}{h^{2}}+s_{1}\left(t^{n+1}\right) .
$$

The pressure correction has a zero Dirichlet boundary condition

$$
\mathrm{q}_{\mathrm{x}}=0
$$

The solution procedure per time step is summarised as follows:

- Step1: Predict the velocity based on the momentum equations (3.98) with pressure and velocity from the previous time step.

- Step2: Solve the pressure correction equation (3.105).

- Step3: Correct the velocity, using (3.102), and pressure, using (3.103).

\subsection{Local grid refinement}

The temperature equation (3.2) is solved with a state-of-the-art flux-limiter scheme in order to preserve the steep temperature gradients that occur in the tube. When large gradients are present, either internally or adjacent to a boundary, more accurate solutions can be obtained by grid refinement. For a uniform grid throughout the computational domain grid refinement can be expensive, particularly in multidimensions. An obvious choice is to use a non-uniform, locally refined, grid. However, uniform grids have several advantages: they can be represented by simple data structures and simple accurate discretisation stencils exist for uniform grids. Instead, the solution can be approximated by using several uniform grids with different mesh sizes that cover different parts of the domain. At least one coarse grid should cover the entire domain. The mesh size of this global grid is chosen in agreement with the smooth behaviour of the solution outside the high-activity regions. Besides a global 

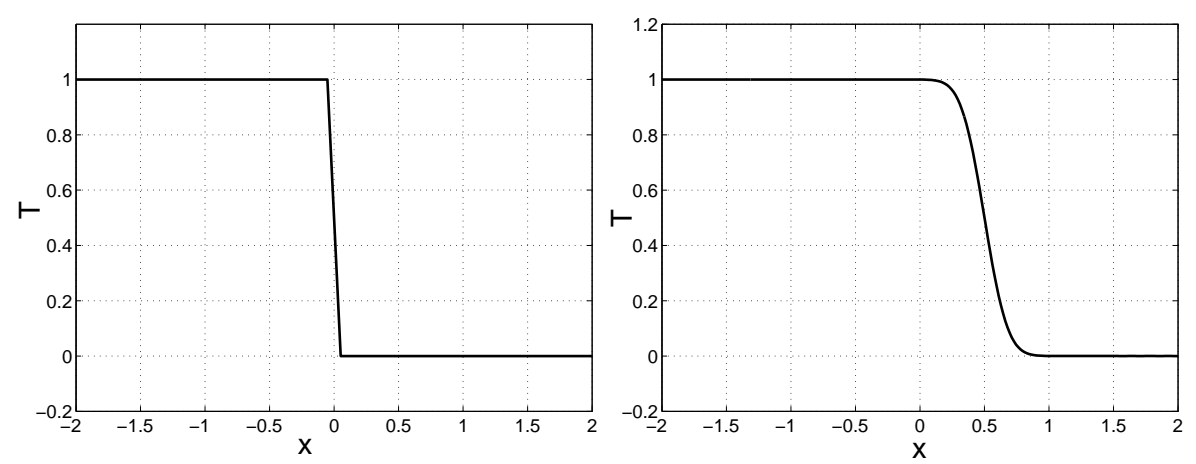

Figure 3.4: Initial $(t=0.0)$ and final $(t=1.0)$ solutions for propagating temperature front with $u=0.5, \epsilon=0.01$.

grid, several local grids can be used which are also uniform. They cover only those parts of the domain that contain the high-activity regions. The mesh size of each of these grids is chosen in agreement with the behaviour of the solution in the corresponding high-activity region. The solution is approximated on the composite grid which is the union of the uniform subgrids. This refinement strategy is known as local uniform grid refinement (LUGR). The technique has been applied to different kinds of PDEs: elliptic [15], [24], hyperbolic [2], [6] and parabolic [17], [72].

\subsubsection{A model problem}

We will explain the basic principles of LUGR in a simple model problem describing the propagation of a diffusing temperature front. The problem has strong similarities with the temperature computation in the pulse tube. The one-dimensional convection-diffusion equation can be written as

$$
\frac{\partial T}{\partial t}=\epsilon \frac{\partial^{2} T}{\partial x^{2}}-u \frac{\partial T}{\partial x}
$$

where the temperature front is convected with a known velocity $u(x, t)>0$ through a fluid of thermal diffusivity $\varepsilon$ in the domain $[-2,2]$. At $t=0$ a sharp front is located at $x=0$, see Figure 3.4(left). The final solution at time $t=1.0$ for $u=0.5, \varepsilon=0.01$ is given in Figure 3.4(right). The following boundary conditions have been used

$$
\mathrm{T}(-2, \mathrm{t})=1.0, \mathrm{~T}(2, \mathrm{t})=0.0 .
$$

To examine the behaviour of the numerical solution we will assume that $u$ and $\varepsilon$ are constants. Then an exact solution can be obtained by separation of variables, see $[18$, p. 306]:

$$
\mathrm{T}_{\text {exact }}(x, t)=0.5-\frac{2}{\pi} \sum_{k=1}^{\infty} \sin \left[(2 k-1) \frac{\pi(x-u t)}{20}\right] \frac{\exp \left[-\varepsilon(2 k-1)^{2} \pi^{2} t / 400\right]}{2 k-1} .
$$




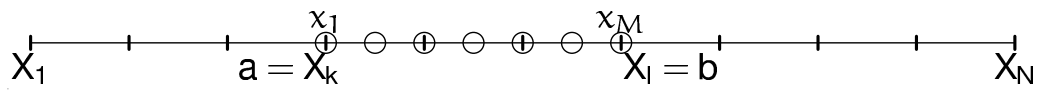

Figure 3.5: Coarse and fine grids with refinement factor $\sigma=2$.

The continuous solution of the boundary value problem (3.111) has a high-activity area. Initially it is located near $x=0$, at $t=1$ it is in the region between $x=0.25$ and $x=0.75$. In the rest of the domain the solution is constant. Due to this fact, a grid with high resolution is needed to accurately represent the solution for $0.25<x<0.75$, whereas a grid with far less resolution is needed in the rest of the domain. We will use a global coarse grid with $N$ points and with step size $H=4.0 /(N-1)$,

$$
\Omega_{[-2,2]}^{\mathrm{H}}:=\left\{X_{i}, i=1, \ldots, N\right\} .
$$

The local fine grid lies on the interval $[a, b]$, such that $a$ and $b$ are always grid points of the coarse grid. The fine grid has $M$ points and step size $h=(b-a) /(M-1)$,

$$
\Omega_{[a, b]}^{h}:=\left\{x_{j}, j=1, \ldots, M\right\} .
$$

We define the refinement factor $\sigma=H / h$. If $a=X_{k}$ and $b=X_{l}$, then the number of fine grid points is $M=\sigma(k-l)+1$. A schematic picture of two grids with refinement factor $\sigma=2$ is given in Figure 3.5. The local region of refinement may be stationary or moving.

\subsubsection{Two-grid LUGR with fixed refinement area}

To advance solution from time $t^{n}$ to $t^{n+1}$ with time step $\Delta t$ the following steps are taken:

- Coarse grid solution

An implicit scheme with central differences for the diffusion term and upwind discretisation for the convection term is used:

$$
\begin{aligned}
& \frac{T_{H}^{n+1}(i)-T_{H}^{n}(i)}{\Delta t}=-\epsilon \frac{T_{H}^{n+1}(i-1)-2 T_{H}^{n+1}(i)+T_{H}^{n+1}(i+1)}{H^{2}}+ \\
&+u \frac{T_{H}^{n+1}(i)-T_{H}^{n+1}(i-1)}{H}, i=2, \ldots, N-1, \\
& T_{H}^{n+1}(1)=1.0, T_{H}^{n+1}(N)=0.0 .
\end{aligned}
$$

The solution $\mathrm{T}_{\mathrm{H}}^{\mathrm{n}+1}$ on the coarse grid is used to define the initial boundary value problem on the fine grid.

- Interpolation 
The fine grid time step $\delta t$ is equal to $\Delta t / \sigma$. To reach the time level $t^{n+1}=t^{n}+\Delta t$ of the coarse grid we have to make intermediate time steps on the fine grid. Dirichlet boundary conditions at the points $a$ and $b$ are prescribed via interpolation of the old $\mathrm{T}_{\mathrm{H}}^{n}$ and new $\mathrm{T}_{\mathrm{H}}^{\mathrm{n}+1}$ coarse grid values. For example, if $\sigma=2$ we will perform two time steps on the fine grid with $\delta t=\Delta t / 2$ and $T_{H}^{n+\frac{1}{2}}=\left(T_{H}^{n+1}+T_{H}^{n}\right) / 2$.

- Fine grid solution $(\sigma=2)$

$$
\begin{aligned}
& \frac{T_{h}^{n+\frac{1}{2}}(i)-T_{h}^{n}(i)}{\delta t}=-\epsilon \frac{T_{h}^{n+\frac{1}{2}}(i-1)-2 T_{h}^{n+\frac{1}{2}}(i)+T_{h}^{n+\frac{1}{2}}(i+1)}{h^{2}}+ \\
& +u \frac{T_{h}^{n+\frac{1}{2}}(i)-T_{h}^{n+\frac{1}{2}}(i-1)}{h}, i=2, \ldots, M-1, \\
& T_{h}^{n+\frac{1}{2}}(1)=T_{H}^{n+\frac{1}{2}}(l), T_{h}^{n+\frac{1}{2}}(M)=T_{H}^{n+\frac{1}{2}}(k), \\
& \frac{T_{h}^{n+1}(i)-T_{h}^{n+\frac{1}{2}}(i)}{\delta t}=-\epsilon \frac{T_{h}^{n+1}(i-1)-2 T_{h}^{n+1}(i)+T_{h}^{n+1}(i+1)}{h^{2}}+ \\
& +u \frac{T_{h}^{n+1}(i)-T_{h}^{n+1}(i-1)}{h}, i=2, \ldots, M-1, \\
& T_{h}^{n+1}(1)=T_{H}^{n+1}(l), T_{h}^{n+1}(M)=T_{H}^{n+1}(k) .
\end{aligned}
$$

- Composite solution

For points of the global coarse grid that lie within the local fine grid, we have found two approximations. We construct a composite solution, using the fine grid solution at these points and the coarse grid solution elsewhere:

$$
T_{H, h}^{n+1}(i)=\left\{\begin{array}{l}
T_{h}^{n+1}(\sigma(i-l)+1) \quad l \leq i \leq k \\
T_{H}^{n+1}(i) \text { otherwise }
\end{array} .\right.
$$

The numerical results of the LUGR method with fixed refinement area are presented in Table 3.3. We consider the composite solution error $\left\|\epsilon_{H, h}\right\|_{\infty}=\left\|T_{\text {exact }}-T_{H, h}\right\|_{\infty}$, using the maximum norm. The table also gives the number of grid points on the coarse and fine meshes. The width of the refinement area is kept constant at 1.5 for all simulations. The fully implicit scheme has no time step stability restriction. But in the comparison of the error for different grids we will use the relation $\Delta t=H / 2$ for the time step on the coarse grid. Table 3.4 shows the results obtained from solving the problem with uniform grids. 


\begin{tabular}{ccccc}
\hline $\mathrm{h}$ & error & $\begin{array}{r}\mathrm{H}=0.2 \\
\text { points } \\
\mathrm{N}+\mathrm{M}\end{array}$ & error & $\begin{array}{c}\mathrm{H}=0.1 \\
\text { points } \\
\mathrm{N}+\mathrm{M}\end{array}$ \\
\hline $\mathrm{H} / 2$ & $1.67 \cdot 10^{-1}$ & $21+15$ & $1.45 \cdot 10^{-1}$ & $41+29$ \\
$\mathrm{H} / 4$ & $1.14 \cdot 10^{-1}$ & $21+29$ & $8.77 \cdot 10^{-2}$ & $41+61$ \\
$\mathrm{H} / 8$ & $7.42 \cdot 10^{-2}$ & $21+57$ & $4.96 \cdot 10^{-2}$ & $41+121$ \\
$\mathrm{H} / 20$ & $3.65 \cdot 10^{-2}$ & $21+141$ & $2.17 \cdot 10^{-2}$ & $41+301$ \\
\hline
\end{tabular}

Table 3.3: Error in temperature $\left\|T_{\text {exact }}-T_{H, h}\right\|_{\infty}$ at time $t=1.0$ with $H=0.2, \tau=0.1$ (left) and $H=0.1, \tau=0.05$ (right), order of fixed refinement $\sigma=2,4,8,20$.

\begin{tabular}{ccc}
\hline $\mathrm{H}$ & error & points \\
\hline \hline 0.05 & $1.55 \cdot 10^{-1}$ & 81 \\
0.025 & $8.43 \cdot 10^{-2}$ & 161 \\
0.0125 & $4.86 \cdot 10^{-2}$ & 321 \\
0.005 & $2.14 \cdot 10^{-2}$ & 801 \\
\hline
\end{tabular}

Table 3.4: Error in temperature $\left\|T_{\text {exact }}-T_{H}\right\|_{\infty}$ at time $t=1.0$ for uniform grids.

\subsubsection{Two-grid LUGR with moving refinement area}

If the region of refinement moves together with the zone of high activity, then we can change its width and reduce the number of fine grid points accordingly. This can be done as follows.

- Coarse grid solution

Solve the system on the coarse grid from $t^{n}$ to $t^{n+1}$ with time step $\Delta t$.

- Regridding strategy

Noting that the fine grid not necessarily moves every time step, decide where the fine grid will be for the present time level. The following regridding criteria can be used. The initial position of the fine grid $[a, b]$ is known, such that $a$ and $b$ are points of the coarse grid: $a=X_{k}, b=X_{l}$. At each time step we move the boundaries at distance $u \Delta t$. As soon as the distance $d=\left|b+u \Delta t-X_{l+1}\right|$ between $b+u \Delta t$ and the next coarse grid point $X_{l+1}$ is less than $\left|b+u \Delta t-X_{l}\right|$, we move the fine grid. 


\begin{tabular}{ccccc}
\hline $\mathrm{h}$ & error & $\begin{array}{r}\mathrm{H}=0.2 \\
\text { points } \\
\mathrm{N}+\mathrm{M}\end{array}$ & error & $\begin{array}{c}\mathrm{H}=0.1 \\
\text { points } \\
\mathrm{N}+\mathrm{M}\end{array}$ \\
\hline \hline $\mathrm{H} / 2$ & $1.74 \cdot 10^{-1}$ & $21+9$ & $1.52 \cdot 10^{-1}$ & $41+15$ \\
$\mathrm{H} / 4$ & $1.18 \cdot 10^{-1}$ & $21+17$ & $8.97 \cdot 10^{-2}$ & $41+29$ \\
$\mathrm{H} / 8$ & $1.13 \cdot 10^{-1}$ & $21+33$ & $5.08 \cdot 10^{-2}$ & $41+57$ \\
$\mathrm{H} / 20$ & $1.05 \cdot 10^{-1}$ & $21+81$ & $2.44 \cdot 10^{-2}$ & $41+141$ \\
\hline
\end{tabular}

Table 3.5: Error in temperature $\left\|T_{\text {exact }}-\mathrm{T}_{\mathrm{H}, \mathrm{h}}\right\|_{\infty}$ at time $\mathrm{t}=1.0$ with $\mathrm{H}=0.2, \tau=$ 0.1 (left) and $\mathrm{H}=0.1, \tau=0.05$ (right), order of moving refinement $\sigma=$ $2,4,8,20$. Initial position of refinement area: $[-0.2,0.4]$.

- Interpolation

If at the present time level $\mathrm{t}^{\mathrm{n}+1}$ regridding is necessary, then go to the previous time level $t^{n}$ and, using interpolation, determine the initial values for the new fine grid.

- Fine grid solution

Solve the system on the fine grid $\sigma$ times with time step $\delta t$.

- Composite solution

Inject the fine grid values in coinciding coarse grid points.

Numerical results of the LUGR method with moving refinement area are presented in Table 3.5. The width of the refinement area is equal to 0.6 (instead of 1.5 in the previous results) for all simulations. By comparing the Tables 3.3, 3.4 and 3.5 we see that the LUGR algorithm with moving refinement area is the most efficient. It provides comparable accuracy with substantially fewer grid points. For instance, to solve the given model problem with the accuracy requirement $\|\epsilon\|_{\infty}=\| T_{\text {exact }}-$ $\mathrm{T}_{\mathrm{H}, \mathrm{h}} \|_{\infty} \leq 3 \cdot 10^{-2}$ we need:

- uniform grid - solution of linear algebraic three-diagonal system of equations of size $801 \times 801$ in each of 400 time steps.

- LUGR with fixed local fine grid - 21 time steps on coarse grid with 41 points and $21^{*} 20$ time steps on fine grid with 301 grid points.

- LUGR with moving local fine grid - 21 time steps on coarse grid with 41 points and $21^{*} 20$ time steps on fine grid with 141 grid points.

From this consideration it is clear that much benefit can be gained from grid refinement moving with large gradients in the solution. 


\section{Numerical solution methods for 2D equations}

For the numerical solution of the resulting system of equations in the two-dimensional case we will use the same techniques as used in the one-dimensional case. We decouple the equations for the computation of the velocity field (2.103) - (2.105) from the energy equation (2.106) and the equation of state (2.107) by using values for temperature and density from the previous time level. The convection-diffusion equation for the gas temperature in the two-dimensional model is combined with a heat conduction equation for the wall temperature. To closely study gas-wall interaction, we employ a non-uniform boundary layer type of grid. The flux-limiter scheme needs to be modified for non-uniform grids. The pressure correction method for the flow computation is specially designed for low-Mach-number compressible flows. Using the continuity equation and the energy equation, we derive an expansion equation or velocity divergence constraint. Our pressure correction scheme is based on this expansion equation and not on the continuity equation, which is different from the common approach in the simulation of compressible flows. The method has close resemblance with incompressible flow computation, except for the non-zero velocity divergence constraint. The newly developed simulation tool, based on the proposed model, is tested on classical problems with known solutions.

\subsection{Temperature computation for the 2D case}

The non-linear convection-diffusion equation for the temperature, assuming constant thermal conductivity ( $k_{g}=1$ in dimensionless form), follows from (2.106)

$$
\frac{\partial \mathrm{T}}{\partial \mathrm{t}}+u \frac{\partial \mathrm{T}}{\partial z}+v \frac{\partial \mathrm{T}}{\partial \mathrm{r}}=\epsilon(\mathrm{t}) \mathrm{T}\left(\frac{\partial^{2} \mathrm{~T}}{\partial \mathrm{r}^{2}}+\frac{1}{\mathrm{r}} \frac{\partial \mathrm{T}}{\partial \mathrm{r}}+\frac{\partial^{2} \mathrm{~T}}{\partial z^{2}}\right)+s_{2}(\mathrm{t}) \mathrm{T},
$$




$$
\begin{aligned}
\epsilon(t) & =\frac{1}{\mathcal{B}(\mathcal{A}+\mathrm{P}(\mathrm{t}))} \frac{1}{\mathrm{Pe}} \ll 1, \\
\mathrm{~s}_{2}(\mathrm{t}) & =\frac{1}{(\mathcal{A}+\mathrm{P}(\mathrm{t}))} \frac{\gamma-1}{\gamma} \frac{\mathrm{dP}}{\mathrm{dt}} .
\end{aligned}
$$

The heat conduction equation for the wall (2.111) reads

$$
\frac{\partial \mathrm{T}_{w}}{\partial \mathrm{t}}=\alpha\left(\frac{\partial^{2} \mathrm{~T}_{w}}{\partial \mathrm{r}^{2}}+\frac{1}{\mathrm{r}} \frac{\partial \mathrm{T}_{w}}{\partial \mathrm{r}}+\frac{\partial^{2} \mathrm{~T}_{w}}{\partial z^{2}}\right) .
$$

The boundary conditions are the following

hot end $(z=0):\left\{\begin{array}{l}T(0, r, t)=T_{H} \text { if } u(0, r, t) \geq 0 \\ T_{w}(0, r, t)=T_{H} \\ u(0, r, t) \frac{\partial T}{\partial z}(0, r, t)=s_{2}(t) T(0, r, t)-\frac{\partial T}{\partial t}(0, r, t) \text { if } u(0, r, t)<0\end{array}\right.$,

cold end $(z=L):\left\{\begin{array}{l}T(L, r, t)=T_{C} \text { if } u(L, r, t) \leq 0 \\ T_{w}(L, r, t)=T_{C} \\ u(L, r, t) \frac{\partial T}{\partial z}(L, r, t)=s_{2}(t) T(L, r, t)-\frac{\partial T}{\partial t}(L, r, t) \text { if } u(L, r, t)>0\end{array}\right.$,

$$
\text { outer wall boundary }\left(r=R_{1}\right): \quad \frac{\partial T_{w}}{\partial r}=0,
$$

$$
\text { inner wall boundary }\left(r=R_{0}\right): \quad \frac{\partial T_{w}}{\partial r}=\beta \frac{\partial T}{\partial r}, T_{w}=T \text {, }
$$

$$
\text { symmetry line }(r=0): \quad \frac{\partial T}{\partial r}=0 .
$$

For the simultaneous numerical solution of the equations (4.1) and (4.4) we introduce two types of grids. The first one, see Figure 4.1(a), is a quasi-uniform grid, defined by

$$
\begin{gathered}
z_{i}=i h_{1}, i=0, \ldots, N_{z}, h_{1}=L / N_{z} . \\
r_{j}=\left\{\begin{array}{l}
j h_{2}, j=0, \ldots, N_{g}-1, h_{2}=R_{0} / N_{g} \text { in the gas domain, } \\
R_{0}+\left(j-N_{g}\right) h_{w}, j=N_{g}, \ldots, N_{r} \text { in the wall domain, } \\
h_{w}=\left(R_{1}-R_{0}\right) / N_{w}, N_{w}=N_{r}-N_{g} .
\end{array}\right.
\end{gathered}
$$

This grid has constant mesh size $h_{1}$ in $z$-direction, constant mesh size $h_{2}$ in r-direction in the gas and constant mesh size $h_{w}$ in r-direction in the wall. Only at the interface ( $r_{j}=R_{0}$ ) the mesh size in $r$-direction changes.

The second grid, see Figure 4.1(b), is uniform with mesh size $h_{1}$ in $z$-direction, uniform with mesh size $h_{w}$ in $r$-direction in the wall and non-uniform in $r$-direction in the gas. The stretching approach for generating structured grids is applied. This method utilises a standard stretching function and allows the generation of grids with node clustering, such that specific regions are resolved accurately without an increase 


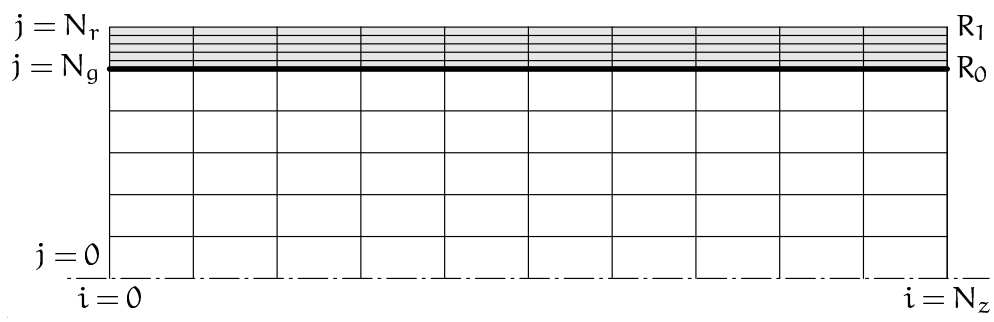

(a) quasi-uniform grid: uniform for the gas and for the wall

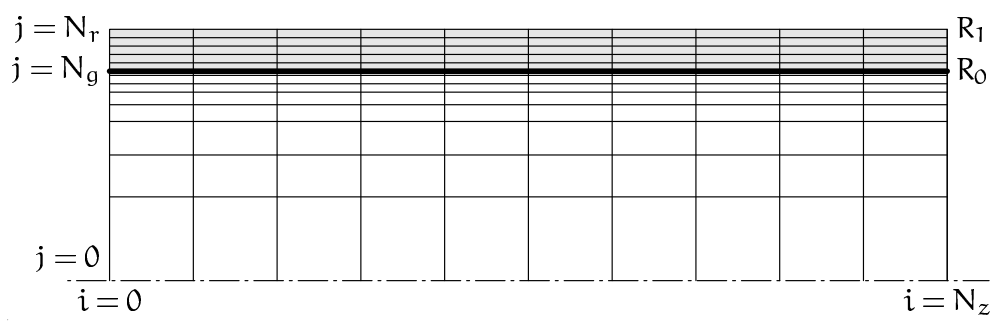

(b) uniform grid for the wall, non-uniform grid for the gas

Figure 4.1: Different types of grids used for the temperature computation.

in the total number of nodes. One effective stretching function, see [18, p. 102], can be constructed as follows

$$
r_{j}=\mathcal{P} s_{j}+(1-\mathcal{P})\left(1-\frac{\tanh \left(\Theta\left(1-s_{j}\right)\right)}{\tanh \Theta}\right)
$$

where $s_{j}$ is a uniform mesh on the unit interval $[0,1] . \mathcal{P}$ and $\Theta$ are parameters providing grid point control: $\mathcal{P}$ gives the slope of the node distribution, $\Theta$ is a damping factor. For $\mathcal{P}=1.8$ and $\Theta=2.0$ the nodes are clustered near $r=1$. For clustering points near the wall at $r=R_{0}$, the grid $\widehat{r}_{j}=R_{0} r_{j}, j=0, \ldots, N_{g}-1$ with variable mesh size $h_{j}=r_{j+1}-r_{j}$ is used. The temperature in the grid point $\left(z_{i}, r_{j}\right)$ at time level $n$ is denoted by $T_{i j}^{n}$. For $0 \leq j \leq N_{g}-1$ this gives the gas temperature and for $N_{g} \leq j \leq N_{r}$ the wall temperature. To find the temperature at time level $n+1$ we use velocities at time level $n$. For the calculation of the gas temperature the same approach is used as in the one-dimensional case: explicit time discretisation and a high-resolution scheme for the convective terms, implicit time discretisation for the diffusive terms and for the source term.

First we present the numerical schemes for the quasi-uniform grid shown in Figure 4.1(a). Then these are extended to grids with non-uniform mesh size. The discretised 
equation (4.1) for the case $u_{i j}^{n}>0, v_{i j}^{n}>0$ is given by

$$
\begin{gathered}
T_{i j}^{n+1}-\epsilon^{n} \tau^{n} T_{j}^{n}\left(\frac{1}{r_{j}} \frac{T_{i j-1}^{n+1}-T_{i j+1}^{n+1}}{2 h_{2}}+\frac{T_{i j-1}^{n+1}-2 T_{i j}^{n+1}+T_{i j+1}^{n+1}}{h_{2}^{2}}+\frac{T_{i-1 j}^{n+1}-2 T_{i j}^{n+1}+T_{i+1 j}^{n+1}}{h_{1}^{2}}\right)- \\
-\tau^{n} s_{2}\left(t^{n+1}\right) T_{i j}^{n+1}=T_{i j}^{n}-c_{i j}^{n}\left(1+\frac{1}{2}\left(1-c_{i j}^{n}\right)\left(\frac{\Psi_{i+\frac{1}{2} j}^{n}}{\theta_{i+\frac{1}{2} j}^{n}}-\Psi_{i-\frac{1}{2} j}^{n}\right)\right)\left(T_{i j}^{n}-T_{i-1 j}^{n}\right)- \\
-d_{i j}^{n}\left(1+\frac{1}{2}\left(1-d_{i j}^{n}\right)\left(\frac{\Psi_{i j+\frac{1}{2}}^{n}}{\theta_{i j+\frac{1}{2}}^{n}}-\Psi_{i j-\frac{1}{2}}^{n}\right)\right)\left(T_{i j}^{n}-T_{i j-1}^{n}\right), \\
i=2, \ldots, N_{z}-2, \quad j=2, \ldots, N_{g}-2 .
\end{gathered}
$$

If $u_{i j}^{n}<0, v_{i j}^{n}>0$, then the right-hand side (rhs) becomes

$$
\begin{aligned}
\text { rhs }= & T_{i j}^{n}-c_{i j}^{n}\left(1-\frac{1}{2}\left(1+c_{i j}^{n}\right)\left(\Psi_{i+\frac{1}{2} j}^{n}-\frac{\Psi_{i-\frac{1}{2} j}^{n}}{\theta_{i-\frac{1}{2} j}^{n}}\right)\right)\left(T_{i+1 j}^{n}-T_{i j}^{n}\right)- \\
& -d_{i j}^{n}\left(1+\frac{1}{2}\left(1-d_{i j}^{n}\right)\left(\frac{\Psi_{i j+\frac{1}{2}}^{n}}{\theta_{i j+\frac{1}{2}}^{n}}-\Psi_{i j-\frac{1}{2}}^{n}\right)\right)\left(T_{i j}^{n}-T_{i j-1}^{n}\right), \\
& i=2, \ldots, N_{z}-2, \quad j=2, \ldots, N_{g}-2 .
\end{aligned}
$$

where $c_{i j}^{n}$ and $d_{i j}^{n}$ are the Courant numbers $c_{i j}^{n}:=\tau^{n} u_{i j}^{n} / h_{1}$ and $d_{i j}^{n}:=\tau^{n} v_{i j}^{n} / h_{2}$. For the cases $u_{i j}^{n}>0, v_{i j}^{n}<0$ and $u_{i j}^{n}<0, v_{i j}^{n}<0$ similar formulae can be derived.

The ratio $\theta_{i+\frac{1}{2} j}^{n}$ is defined by

$$
\theta_{i+\frac{1}{2} j}^{n}:=\left\{\begin{array}{l}
\frac{T_{i j}^{n}-T_{i-1 j}^{n}}{T_{i+1 j}^{n}-T_{i j}^{n}} \text { if } u_{i j}^{n}>0 \\
\frac{T_{i+2 j}^{n}-T_{i+1 j}^{n}}{T_{i+1 j}^{n}-T_{i j}^{n}} \text { if } u_{i j}^{n}<0
\end{array} .\right.
$$

The ratios $\theta_{i-\frac{1}{2}}^{n}, \theta_{i j+\frac{1}{2}}^{n}$ and $\theta_{i j-\frac{1}{2}}^{n}$ are constructed analogously. Computing $T_{i j}^{n+1}$ with the formulae (4.13) and (4.14) requires nine-point stencils. For the points next to the gas boundaries $\left(i=1, i=N_{z}-1, j=1, j=N_{g}-2\right)$ a standard upwind scheme for the convection term is used.

In the wall part of the computational domain we solve the heat conduction equation (4.4) implicitly,

$$
\begin{aligned}
\frac{T_{i j}^{n+1}-T_{i j}^{n}}{\tau^{n}}= & \alpha\left(\frac{1}{r_{j}} \frac{T_{i j-1}^{n+1}-T_{i j+1}^{n+1}}{2 h_{w}}+\frac{T_{i j-1}^{n+1}-2 T_{i j}^{n+1}+T_{i j+1}^{n+1}}{h_{w}^{2}}+\frac{T_{i-1 j}^{n+1}-2 T_{i j}^{n+1}+T_{i+1 j}^{n+1}}{h_{1}^{2}}\right), \\
& i=1, \ldots, N_{z}-1, \quad j=N_{g}+1, \ldots, N_{r}-1 .
\end{aligned}
$$


At the interface $\left(j=N_{g}\right)$ we use the following approximation

$$
\frac{T_{i j+1}^{n+1}-T_{i j}^{n+1}}{r_{j+1}-r_{j}}=\beta \frac{T_{i j}^{n+1}-T_{i j-1}^{n+1}}{r_{j}-r_{j-1}}, i=1, \ldots, N_{z}-1 .
$$

We choose the time step according to the CFL (Courant-Friedrichs-Lewy) stability condition $\left|c_{i j}^{\mathfrak{n}}\right| \leq 1,\left|d_{i j}^{\mathfrak{n}}\right| \leq 1$, i.e.

$$
\tau^{n} \leq \min \left\{h_{1} / \max _{i j}\left|u_{i j}^{n}\right|, h_{2} / \max _{i j}\left|v_{i j}^{n}\right|\right\} .
$$

In general, finite-difference numerical schemes can easily be extended to non-uniform meshes, but non-trivial modifications are required for the flux-limiters. In [7] it was shown that on irregular meshes the standard limiters may need to be modified to preserve positivity of the scheme. In our case, the ratios $\theta_{i j+\frac{1}{2}}^{n}$ and $\theta_{i j-\frac{1}{2}}^{n}$ will change because the mesh is non-uniform in radial direction. According to their definition, they give the ratio of the upwind and central difference approximations of $\partial \mathrm{T} / \partial \mathrm{r}$ in $r_{j+\frac{1}{2}}$ and $r_{j-\frac{1}{2}}$,

$$
\begin{aligned}
& \theta_{i j+\frac{1}{2}}^{n}:=\left\{\begin{array}{l}
\frac{T_{i j}^{n}-T_{i j-1}^{n}}{T_{i j+1}^{n}-T_{i j}^{n}} \frac{r_{j+1}-r_{j}}{r_{j}-r_{j-1}} \text { if } v_{i j}^{n}>0 \\
\frac{T_{i j+2}^{n}-T_{i j+1}^{n}}{T_{i j+1}^{n}-T_{i j}^{n}} \frac{r_{j+1}-r_{j}}{r_{j+2}-r_{j+1}} \text { if } v_{i j}^{n}<0
\end{array},\right. \\
& \theta_{i j-\frac{1}{2}}^{n}:=\left\{\begin{array}{l}
\frac{T_{i j-1}^{n}-T_{i j-2}^{n}}{T_{i j}^{n}-T_{i j-1}^{n}} \frac{r_{j}-r_{j-1}}{r_{j-1}-r_{j-2}} \text { if } v_{i j}^{n}>0 \\
\frac{T_{i j+1}^{n}-T_{i j}^{n}}{T_{i j}^{n}-T_{i j-1}^{n}} \frac{r_{j}-r_{j-1}}{r_{j+1}-r_{j}} \text { if } v_{i j}^{n}<0
\end{array}\right.
\end{aligned}
$$

The right-hand side of scheme (4.13) for $u_{i j}^{n}>0, v_{i j}^{n}>0$ is changed to

$$
\begin{aligned}
\text { rhs }= & T_{i j}^{n}-c_{i j}^{n}\left(1+\frac{1}{2}\left(1-c_{i j}^{n}\right)\left(\frac{\Psi_{i+\frac{1}{2} j}^{n}}{\theta_{i+\frac{1}{2} j}^{n}}-\Psi_{i-\frac{1}{2} j}^{n}\right)\right)\left(T_{i j}^{n}-T_{i-1 j}^{n}\right)- \\
& -d_{i j}^{n}\left(1+\frac{1}{2}\left(1-d_{i j}^{n}\right)\left(\frac{\Psi_{i j+\frac{1}{2}}^{n}}{\theta_{i j+\frac{1}{2}}^{n}} \frac{r_{j+1}-r_{j}}{r_{j}-r_{j-1}}-\Psi_{i j-\frac{1}{2}}^{n}\right)\right)\left(T_{i j}^{n}-T_{i j-1}^{n}\right),\left(4,2, \ldots, N_{g}-2 .\right.
\end{aligned}
$$

A sufficient condition for the high-resolution scheme to be TVD, see [49], is

$$
\left|\frac{\Psi_{i j+\frac{1}{2}}^{n}}{\theta_{i j+\frac{1}{2}}^{n}} \frac{r_{j+1}-r_{j}}{r_{j}-r_{j-1}}-\Psi_{i j-\frac{1}{2}}^{n}\right| \leq 2 .
$$




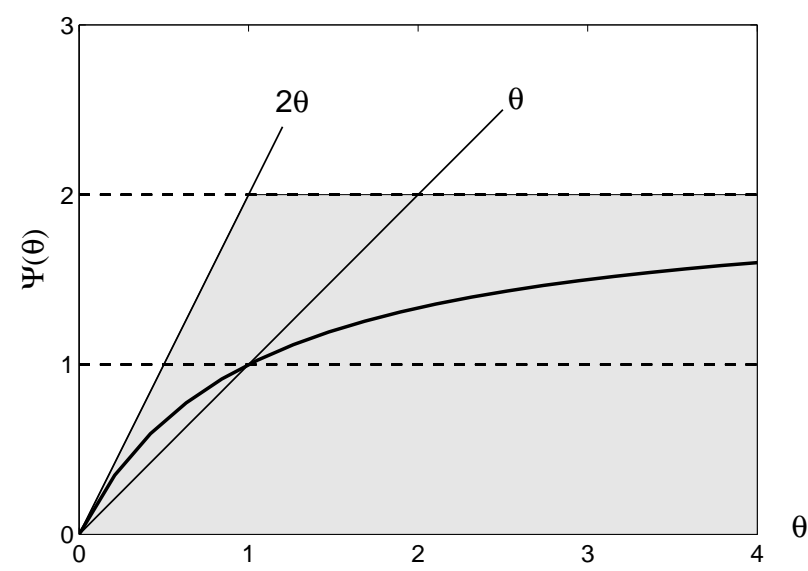

Figure 4.2: TVD region (gray) and Van Leer limiter (bold line).

If we denote the mesh ratio by $q$, the inequality (4.22) becomes

$$
\left|\frac{\Psi\left(\theta_{1}\right)}{\theta_{1}} q-\Psi\left(\theta_{2}\right)\right| \leq 2 \text { with } q=\frac{r_{j+1}-r_{j}}{r_{j}-r_{j-1}} \text { for all } \theta_{1}, \theta_{2} .
$$

For all $\theta_{1}, \theta_{2}>0$, the inequality in (4.23) holds, if $\Psi(\theta)$ satisfies

$$
0 \leq \Psi(\theta) \leq \min \left(2, \frac{2}{\mathrm{q}} \theta\right) \text { for all } \theta>0 .
$$

As can be seen from Figure 4.2, the Van Leer limiter

$$
\Psi(\theta)=\frac{\theta+|\theta|}{1+|\theta|}
$$

satisfies the condition (4.24) if $\mathrm{q} \leq 1$. The modified Van Leer limiter, defined by

$$
\Psi(\theta)=\frac{\theta+|\theta|}{1+\max (1,|\theta|)},
$$

differs from Van Leer limiter in the region $0<\theta<1$ and satisfies (4.24) if $\mathrm{q} \leq 2$.

Let us consider the case of negative radial velocity. The right-hand side of equation (4.13) for $u_{i j}^{n}>0, v_{i j}^{n}<0$ is

$$
\begin{aligned}
\text { rhs }= & T_{i j}^{n}-c_{i j}^{n}\left(1+\frac{1}{2}\left(1-c_{i j}^{n}\right)\left(\frac{\Psi_{i+\frac{1}{2} j}^{n}}{\theta_{i+\frac{1}{2} j}^{n}}-\Psi_{i-\frac{1}{2} j}^{n}\right)\right)\left(T_{i j}^{n}-T_{i-1 j}^{n}\right)- \\
& -d_{i j}^{n}\left(1-\frac{1}{2}\left(1+d_{i j}^{n}\right)\left(\Psi_{i j+\frac{1}{2}}^{n}-\frac{\Psi_{i j-\frac{1}{2}}^{n}}{\theta_{i j-\frac{1}{2}}^{n}} \frac{r_{j}-r_{j-1}}{r_{j+1}-r_{j}}-\right)\right)\left(T_{i j+1}^{n}-T_{i j}^{n}\right) \\
& i=2, \ldots, N_{z}-2, \quad j=2, \ldots, N_{g}-2 .
\end{aligned}
$$


The TVD condition is now

$$
\left|\Psi_{i j+\frac{1}{2}}^{n}-\frac{\Psi_{i j-\frac{1}{2}}^{n}}{\theta_{i j-\frac{1}{2}}^{n}} \frac{r_{j}-r_{j-1}}{r_{j+1}-r_{j}}\right| \leq 2 .
$$

Using the notation $\mathrm{q}$ for the mesh ratio, the inequality (4.28) reads

$$
\left|\Psi_{i j+\frac{1}{2}}^{n}-\frac{\Psi_{i j-\frac{1}{2}}^{n}}{\theta_{i j-\frac{1}{2}}^{n}} \frac{1}{q}\right| \leq 2 \text {, for all } \theta_{1}, \theta_{2} .
$$

For all $\theta_{1}, \theta_{2}>0$, the inequality in (4.29) holds, if $\Psi(\theta)$ satisfies

$$
0 \leq \Psi(\theta) \leq \min (2,2 q \theta) \text { for all } \theta>0
$$

The Van Leer limiter satisfies the condition (4.30) if $q>1$. The modified Van Leer limiter satisfies (4.30) if $\mathrm{q}>1 / 2$.

This consideration shows that for the Van Leer limiter the TVD condition requires $\mathrm{q} \leq 1$ for positive velocities and $\mathrm{q}>1$ for negative velocities. So it cannot be used when a stretched grid is employed. The modified Van Leer limiter satisfies the TVD condition, if the mesh ratio satisfies $1 / 2<q<2$. The minmod limiter, given by (3.16), can be used on non-uniform grids without modification, but its resolution of discontinuities is not so good as with the Van Leer limiter [26, p. 542].

\subsection{Pressure correction algorithm for the 2D case}

After the calculation of temperature $T^{n+1}$ and density $\rho^{n+1}$, we have to compute velocities $u^{n+1}, v^{n+1}$ and pressure $p^{n+1}$. The momentum equations (2.103), (2.104) and the constraint (2.105) with constant thermal conductivity are

$$
\begin{gathered}
\rho\left(\frac{\partial u}{\partial t}+u \frac{\partial u}{\partial z}+v \frac{\partial u}{\partial r}\right)=-\frac{\partial p}{\partial z}+\frac{1}{\operatorname{Re}}\left[\frac{\partial^{2} u}{\partial r^{2}}+\frac{1}{r} \frac{\partial u}{\partial r}+\frac{4}{3} \frac{\partial^{2} u}{\partial z^{2}}+\frac{1}{3} \frac{1}{r} \frac{\partial v}{\partial r}+\frac{1}{3} \frac{\partial}{\partial z}\left(\frac{\partial v}{\partial r}\right)\right], \\
\rho\left(\frac{\partial v}{\partial t}+u \frac{\partial v}{\partial z}+v \frac{\partial v}{\partial r}\right)=-\frac{\partial p}{\partial r}+\frac{1}{\operatorname{Re}}\left[\frac{4}{3} \frac{\partial^{2} v}{\partial r^{2}}+\frac{\partial^{2} v}{\partial z^{2}}+\frac{2}{3} \frac{1}{r} \frac{\partial v}{\partial r}-\frac{2}{3} \frac{1}{r} \frac{\partial u}{\partial z}+\frac{1}{3} \frac{\partial}{\partial r}\left(\frac{\partial u}{\partial z}\right)\right], \\
\frac{\partial u}{\partial z}+\frac{\partial v}{\partial r}+\frac{v}{r}=s_{1}(t)+\epsilon(t)\left(\frac{1}{r} \frac{\partial T}{\partial r}+\frac{\partial^{2} T}{\partial r^{2}}+\frac{\partial^{2} T}{\partial z^{2}}\right), \\
s_{1}(t)=-\frac{1}{\gamma} \frac{1}{(\mathcal{A}+\mathrm{P})} \frac{\mathrm{dP}}{\mathrm{dt}} .
\end{gathered}
$$

The boundary conditions are

$$
\text { hot end }(z=0): \quad u=u_{H}(t), v=0,
$$




$$
\begin{aligned}
\operatorname{cold} \text { end }(z=L): & p=0, v=0, \\
\text { wall boundary }\left(r=R_{0}\right): & u=0, v=0, p=0, \\
\text { symmetry line }(r=0): & \frac{\partial u}{\partial r}=0, v=0, \frac{\partial p}{\partial r}=0 .
\end{aligned}
$$

ALGORITHM 2 as described in Section 3.1 is now applied. A variable time step $\tau^{n}$ is used, which corresponds to the time step used in the temperature computation and which is defined by (4.18).

prediction step:

$$
\begin{gathered}
\frac{u_{i j}^{*}-u_{i j}^{n}}{\tau^{n}}=H\left(u_{i j}^{*}\right)-\frac{1}{\rho_{i j}^{n+1}} \frac{p_{i j}^{n}-p_{i-1 j}^{n}}{h_{1}}, \\
\frac{v_{i j}^{*}-v_{i j}^{n}}{\tau^{n}}=G\left(v_{i j}^{*}\right)-\frac{1}{\rho_{i j}^{n+1}} \frac{p_{i j}^{n}-p_{i j-1}^{n}}{h_{2}}, \\
H\left(u_{i j}^{*}\right)=-u_{i j}^{n} \frac{u_{i j}^{*}-u_{i-1 j}^{*}}{h_{1}}-u_{i j}^{n} \frac{u_{i+1 j}^{*}-u_{i j}^{*}}{h_{1}}-v_{i j}^{n} \frac{u_{i j}^{*}-u_{i j-1}^{*}}{h_{2}}-v_{i j}^{n} \frac{u_{i j+1}^{*}-u_{i j}^{*}}{h_{2}}+ \\
+\frac{1}{\operatorname{Re}} \frac{1}{\rho_{i j}^{n+1}}\left[\frac{u_{i j+1}^{*}-2 u_{i j}^{*}+u_{i j-1}^{*}}{h_{2}^{2}}+\frac{1}{r_{j}} \frac{u_{i j+1}^{*}-u_{i j-1}^{*}}{2 h_{2}}+\frac{4}{3} \frac{u_{i+1 j}^{*}-2 u_{i j}^{*}+u_{i-1 j}^{*}}{h_{1}^{2}}\right]+ \\
+\frac{1}{\operatorname{Re}} \frac{1}{\rho_{i j}^{n+1}}\left[\frac{1}{3} \frac{1}{r_{j}} \frac{v_{i j+1}^{n}-v_{i j-1}^{n}}{2 h_{2}}+\frac{1}{3} \frac{v_{i+1 j+1}^{n}-v_{i-1 j+1}^{n}-v_{i+1 j-1}^{n}+v_{i-1 j-1}^{n}}{4 h_{1} h_{2}}\right], \quad(4.41) \\
G\left(v_{i j}^{*}\right)=-u_{i j}^{n} \frac{v_{i j}^{*}-v_{i-1 j}^{*}}{h_{1}}-u_{i j}^{n}-\frac{v_{i+1 j}^{*}-v_{i j}^{*}}{h_{1}}-v_{i j}^{n} \frac{v_{i j}^{*}-v_{i j-1}^{*}}{h_{2}}-v_{i j-}^{n} \frac{v_{i j+1}^{*}-v_{i j}^{*}}{h_{2}}+ \\
+\frac{1}{\operatorname{Re}} \frac{1}{\rho_{i j}^{n+1}}\left[\frac{4}{3} \frac{v_{i j+1}^{*}-2 v_{i j}^{*}+v_{i j-1}^{*}}{h_{2}^{2}}+\frac{2}{3} \frac{1}{r_{j}} \frac{v_{i j+1}^{*}-v_{i j-1}^{*}}{2 h_{2}}+\frac{v_{i+1 j}^{*}-2 v_{i j}^{*}+v_{i-1 j}^{*}}{h_{1}^{2}}\right]+ \\
+\frac{1}{\operatorname{Re}} \frac{1}{\rho_{i j}^{n+1}}\left[-\frac{2}{3} \frac{1}{r_{j}} \frac{u_{i+1 j}^{n}-u_{i-1 j}^{n}}{2 h_{1}}+\frac{1}{3} \frac{u_{i+1 j+1}^{n}-u_{i-1 j+1}^{n}-u_{i+1 j-1}^{n}+u_{i-1 j-1}^{n}}{4 h_{1} h_{2}}\right] .
\end{gathered}
$$

correction step:

$$
\begin{aligned}
& \frac{u_{i j}^{n+1}-u_{i j}^{n}}{\tau^{n}}=H\left(u_{i j}^{*}\right)-\frac{1}{\rho_{i j}^{n+1}} \frac{p_{i j}^{n+1}-p_{i-1 j}^{n+1}}{h_{1}}, \\
& \frac{v_{i j}^{n+1}-v_{i j}^{n}}{\tau^{n}}=G\left(v_{i j}^{*}\right)-\frac{1}{\rho_{i j}^{n+1}} \frac{p_{i j}^{n+1}-p_{i j-1}^{n+1}}{h_{2}} .
\end{aligned}
$$

Subtraction of the equations (4.39) and (4.40) from (4.43) and (4.44), respectively, gives

$$
u_{i j}^{n+1}=u_{i j}^{*}-\frac{\tau^{n}}{h_{1}} \frac{1}{\rho_{i j}^{n+1}} \frac{1}{C_{i j}^{n}}\left[q_{i j}-q_{i-1 j}\right],
$$




$$
\begin{gathered}
v_{i j}^{n+1}=v_{i j}^{*}-\frac{\tau^{n}}{h_{2}} \frac{1}{\rho_{i j}^{n+1}} \frac{1}{D_{i j}^{n}}\left[q_{i j}-q_{i j-1}\right], \\
C_{i j}^{n}=1-\tau^{n} A_{i j}^{n} \text { with } A_{i j}^{n}=-\frac{1}{h_{1}}\left|u_{i j}^{n}\right|-\frac{1}{h_{2}}\left|v_{i j}^{n}\right|-\frac{1}{\operatorname{Re}} \frac{1}{\rho_{i j}^{n+1}}\left(\frac{2}{h_{2}^{2}}+\frac{4}{3} \frac{2}{h_{1}^{2}}\right), \\
D_{i j}^{n}=1-\tau^{n} B_{i j}^{n} \text { with } B_{i j}^{n}=-\frac{1}{h_{1}}\left|u_{i j}^{n}\right|-\frac{1}{h_{2}}\left|v_{i j}^{n}\right|-\frac{1}{\operatorname{Re}} \frac{1}{\rho_{i j}^{n+1}}\left(\frac{4}{3} \frac{2}{h_{2}^{2}}+\frac{2}{h_{1}^{2}}\right) .
\end{gathered}
$$

pressure correction equation:

Equation (4.33) is approximated by

$$
\begin{gathered}
\frac{u_{i+1 j}^{n+1}-u_{i j}^{n+1}}{h_{1}}+\frac{v_{i j+1}^{n+1}-v_{i j}^{n+1}}{h_{2}}+\frac{v_{i j}^{n+1}}{r_{j}}=F_{i j}^{n+1}, \\
F_{i j}^{n+1}=s_{1}\left(t^{n+1}\right)+ \\
\epsilon^{n+1}\left[\frac{1}{r_{j}} \frac{T_{i j-1}^{n+1}+T_{i j+1}^{n+1}}{2 h_{2}}+\frac{T_{i j-1}^{n+1}-2 T_{i j}^{n+1}+T_{i j+1}^{n+1}}{h_{2}^{2}}+\frac{T_{i-1 j}^{n+1}-2 T_{i j}^{n+1}+T_{i+1 j}^{n+1}}{h_{1}^{2}}\right] .
\end{gathered}
$$

Substitution of (4.45) and (4.47) gives

$$
\begin{gathered}
-\frac{\tau^{n}}{h_{1}^{2}} \frac{1}{C_{i+1 j}^{n}}\left[q_{i+1 j}-q_{i j}\right]+\frac{\tau^{n}}{h_{1}^{2}} \frac{1}{C_{i j}^{n}}\left[q_{i j}-q_{i-1 j}\right]-\frac{\tau^{n}}{h_{2}^{2}} \frac{1}{D_{i j+1}^{n}}\left[q_{i j+1}-q_{i j}\right]+ \\
\frac{\tau^{n}}{h_{2}^{2}} \frac{1}{D_{i j}^{n}}\left[q_{i j}-q_{i j-1}\right]-\frac{\tau^{n}}{h_{2}^{2}} \frac{1}{D_{i j}^{n}} \frac{1}{r_{j}}\left[q_{i j}-q_{i j-1}\right]=F_{i j}^{n+1}-\frac{u_{i+1 j}^{*}-u_{i j}^{*}}{h_{1}}-\frac{v_{i j+1}^{*}-v_{i j}^{*}}{h_{2}}-\frac{v_{i j}^{*}}{r_{j}} .
\end{gathered}
$$

The equations (4.39) - (4.49) are valid in the interior points of the computational domain. Boundary points are considered separately.

$$
\begin{aligned}
& \text { Left boundary }(z=0): i=0, j=0, \ldots, N_{g}-1 \\
& u_{0 j}^{n+1} \text { given, } v_{0 j}^{n+1}=0 \text {, } \\
& \frac{u_{1 j}^{n+1}-u_{0 j}^{n+1}}{h_{1}}=F_{1 j}^{n+1}, \quad u_{1 j}^{n+1}=u_{1 j}^{*}-\frac{\tau^{n}}{h_{1}} \frac{1}{\rho_{1 j}^{n+1}} \frac{1}{C_{1 j}^{n}}\left[q_{1 j}-q_{0 j}\right] \text {, } \\
& -\frac{\tau^{n}}{h_{1}^{2}} \frac{1}{\rho_{1 j}^{n+1}} \frac{1}{C_{1 j}^{n}}\left[q_{1 j}-q_{0 j}\right]=F_{1 j}^{n+1}-\frac{u_{1 j}^{*}-u_{0 j}^{n+1}}{h_{1}} .
\end{aligned}
$$


Right boundary $(z=\mathrm{L}): i=\mathrm{N}_{\mathrm{x}}, \mathrm{j}=0, \ldots, \mathrm{N}_{\mathrm{g}}-1$

Using the boundary condition for the radial velocity $v=0$, a Neumann boundary condition for the axial velocity in the predictor step is derived from the constraint equation

$$
\frac{u_{N_{x} j}^{*}-u_{N_{x}-1 j}^{*}}{h_{1}}=F_{N_{x} j}^{n+1}
$$

The correction step is the same as for the interior points

$$
u_{N_{x} j}^{n+1}=u_{N_{x} j}^{*}-\frac{\tau^{n}}{h_{1}} \frac{1}{\rho_{N_{x} j}^{n+1}} \frac{1}{C_{N_{x} j}^{n}}\left[q_{N_{x} j}-q_{N_{x}-1 j}\right], v_{N_{x} j}^{n+1}=0, q_{N_{x} j}=0 .
$$

Wall boundary $\left(r=R_{0}\right): i=0, \ldots, N_{x}, j=N_{g}$

$$
u_{i N_{g}}^{n+1}=0, v_{i N_{g}}^{n+1}=0, q_{i N_{g}}=0
$$

$$
\text { Symmetry boundary }(r=0): i=1, \ldots, N_{x}-1, j=0
$$

Applying the conditions $v=0, \partial u / \partial r=0, \partial \mathrm{T} / \partial r=0$ and using the following asymptotic relations at $r=0$ for removing singularities

$$
\frac{1}{r} \frac{\partial u}{\partial r} \rightarrow \frac{\partial^{2} u}{\partial r^{2}}, \frac{v}{r} \rightarrow \frac{\partial v}{\partial r}, \frac{1}{r} \frac{\partial \mathrm{T}}{\partial r} \rightarrow \frac{\partial^{2} \mathrm{~T}}{\partial r^{2}},
$$

the equations (4.31) - (4.33) become

$$
\begin{gathered}
\rho\left(\frac{\partial u}{\partial t}+u \frac{\partial u}{\partial z}\right)=-\frac{\partial p}{\partial z}+\frac{1}{\operatorname{Re}}\left[2 \frac{\partial^{2} u}{\partial r^{2}}+\frac{4}{3} \frac{\partial^{2} u}{\partial z^{2}}+\frac{1}{3} \frac{\partial^{2} v}{\partial r^{2}}+\frac{1}{3} \frac{\partial}{\partial z}\left(\frac{\partial v}{\partial r}\right)\right], \\
\frac{\partial u}{\partial z}+2 \frac{\partial v}{\partial r}=s_{1}(t)+\epsilon(t)\left(2 \frac{\partial^{2} T}{\partial r^{2}}+\frac{\partial^{2} T}{\partial z^{2}}\right) .
\end{gathered}
$$

The pressure correction steps are then

$$
\begin{gathered}
\frac{u_{i 0}^{*}-u_{i 0}^{n}}{\tau^{n}}=H\left(u_{i 0}^{*}\right)-\frac{p_{i 0}^{n}-p_{i-10}^{n}}{h_{1}}, \\
H\left(u_{i 0}^{*}\right)=-u_{i 0+}^{n} \frac{u_{i 0}^{*}-u_{i-10}^{*}}{h_{1}}-u_{i 0_{-}}^{n} \frac{u_{i+10}^{*}-u_{i 0}^{*}}{h_{1}}+ \\
+\frac{1}{\operatorname{Re}}\left(4 \frac{u_{i 1}^{*}-u_{i 0}^{*}}{h_{2}^{2}}+\frac{4}{3} \frac{u_{i+10}^{*}-2 u_{i 0}^{*}+u_{i-10}^{*}}{h_{1}^{2}}+\frac{2}{3} \frac{v_{i 1}^{n}}{h_{2}^{2}}+\frac{1}{6} \frac{v_{i+11}^{n}-v_{i-11}^{n}}{h_{1} h_{2}}\right),
\end{gathered}
$$




$$
\begin{gathered}
\frac{u_{i+10}^{n+1}-u_{i 0}^{n+1}}{h_{1}}+\frac{2 v_{i 1}^{n+1}}{h_{2}}=F_{i 0}^{n+1} \\
-\frac{\tau^{n}}{h_{1}^{2}} \frac{1}{C_{i+10}^{n}} q_{i+10}-\frac{\tau^{n}}{h_{1}^{2}} \frac{1}{C_{i 0}^{n}} q_{i-10}-\frac{\tau^{n}}{h_{2}^{2}} \frac{2}{D_{i 1}^{n}} q_{i 1}+\frac{\tau^{n}}{h_{1}^{2}}\left[\frac{1}{C_{i+10}^{n}}+\frac{1}{C_{i 0}^{n}}\right] q_{i 0}+\frac{\tau^{n}}{h_{2}^{2}} \frac{2}{D_{i 1}^{n}} q_{i 0}= \\
F_{i 0}^{n+1}-\frac{u_{i+10}^{*}-u_{i 0}^{*}}{h_{1}}-\frac{2 v_{i 1}^{*}}{h_{2}}, \\
F_{i 0}^{n+1}=s_{1}\left(t^{n+1}\right)+\epsilon^{n+1}\left[2 \frac{T_{i 1}^{n+1}-T_{i 0}^{n+1}}{h_{2}^{2}}+\frac{T_{i-10}^{n+1}-2 T_{i 0}^{n+1}+T_{i+10}^{n+1}}{h_{1}^{2}}\right]
\end{gathered}
$$

\subsection{Numerical examples}

The described methods have been implemented in a $\mathrm{C}++$ code. Before performing the pulse tube simulations, the credibility of the code needs to be assessed. For the code verification the following problems have been chosen: Hagen-Poiseuille flow, starting flow, flow due to an oscillating pressure gradient, backward facing step flow and two Graetz problems. The first three examples are well known problems with analytical solutions. The backward facing step flow is a benchmark problem widely used in computational fluid dynamics, a lot of experimental and numerical information about this type of flow is available. The Graetz problems allow the assessment of the temperature computations. Analytical solutions are available for the temperature distribution in fully developed pipe flow with constant wall heat flux or with constant wall temperature.

\subsubsection{Hagen-Poiseuille flow in a circular pipe}

Laminar incompressible flow in a circular pipe driven by a steady pressure gradient is perhaps the most celebrated viscous flow with a known analytical solution. It was first studied by Hagen (1839) and Poiseuille (1840). The governing equation for fully developed pipe flow is the axial momentum equation in cylindrical coordinates. If the flow direction is assumed to be parallel to the $z$ axis, then the radial component of the velocity vanishes. The equation of continuity gives then $\partial u / \partial z=0$, so that $u$ is independent of $z$. With this assumption of one-directional flow, the momentum equation has the form

$$
\frac{\partial p}{\partial z}=\mu\left[\frac{\partial^{2} u}{\partial r^{2}}+\frac{1}{r} \frac{\partial u}{\partial r}\right],
$$

with the boundary conditions being $u=0$ at $r=R_{0}$ and $\partial u / \partial r=0$ at $r=0$. Integration of equation (4.63) gives the velocity distribution

$$
u(r)=-\frac{1}{4 \mu} \frac{\partial p}{\partial z}\left(R_{0}^{2}-r^{2}\right)
$$




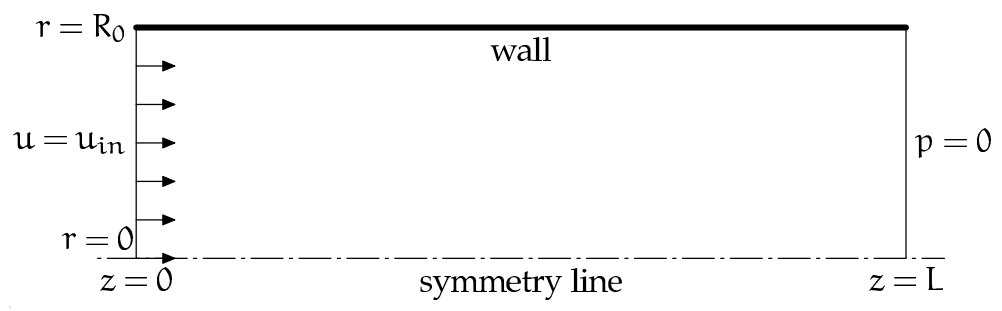

Figure 4.3: Configuration of the test problem: developing Hagen-Poiseuille flow .

The maximum velocity $u_{\max }$ is in the centre, the mean (cross-sectional averaged) velocity is $u_{a v}=u_{\max } / 2$, hence

$$
u_{\mathrm{a} v}=-\frac{\mathrm{R}_{0}^{2}}{8 \mu} \frac{\partial p}{\partial z} .
$$

Let us consider the following test problem: a circular pipe with imposed plug (radially uniform) flow at the inlet and known pressure at the exit. Figure 4.3 illustrates the axisymmetrical configuration of the problem. The governing equations are the conservation laws for incompressible viscous flow in cylindrical coordinates:

$$
\begin{gathered}
\rho\left(\frac{\partial u}{\partial t}+u \frac{\partial u}{\partial z}+v \frac{\partial u}{\partial r}\right)=-\frac{\partial p}{\partial z}+\mu\left[\frac{\partial^{2} u}{\partial r^{2}}+\frac{1}{r} \frac{\partial u}{\partial r}\right], \\
\rho\left(\frac{\partial v}{\partial t}+u \frac{\partial v}{\partial z}+v \frac{\partial v}{\partial r}\right)=-\frac{\partial p}{\partial r}+\mu\left[\frac{\partial^{2} v}{\partial r^{2}}+\frac{1}{r} \frac{\partial v}{\partial r}-\frac{v}{r^{2}}\right], \\
\frac{\partial u}{\partial z}+\frac{\partial v}{\partial r}+\frac{v}{r}=0 .
\end{gathered}
$$

The parameters of the test problem are: length of the tube $L=3[\mathrm{~m}]$, radius $R_{0}=$ $1[\mathrm{~m}]$, viscosity $\mu=1\left[\mathrm{~kg} \cdot \mathrm{m}^{-1} \cdot \mathrm{s}^{-1}\right]$, density $\rho=12\left[\mathrm{~kg} \cdot \mathrm{m}^{-3}\right]$ and inflow velocity $u_{\text {in }}=1 / 24\left[\mathrm{~m} \cdot \mathrm{s}^{-1}\right]$.

The boundary and initial conditions are defined as follows:

$$
\begin{aligned}
\text { inflow }(z=0): & u=u_{i n}, v=0, \\
\operatorname{outflow}(z=L): & v=0, p=0, \\
\text { wall }\left(r=R_{0}\right): & u=0, v=0, \\
\text { symmetry line }(r=0): & \frac{\partial p}{\partial r}=0, \frac{\partial u}{\partial r}=0, v=0, \\
\text { initial conditions }(t=0): & u=u_{i n}, v=0, p=0 .
\end{aligned}
$$




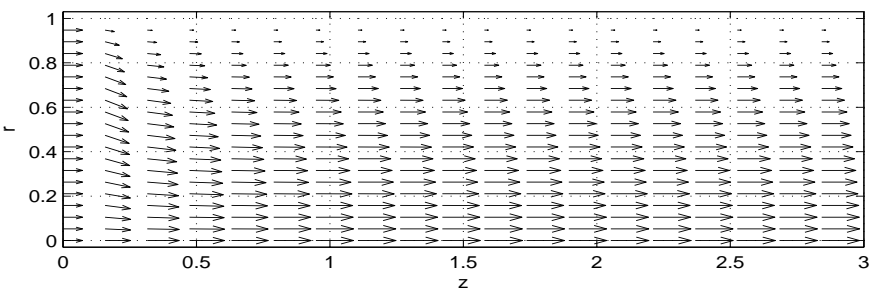

Figure 4.4: Velocity field for developing Hagen-Poiseuille flow.

The non-dimensional equations (4.66) - (4.68) read

$$
\begin{gathered}
\frac{\partial u}{\partial t}+u \frac{\partial u}{\partial z}+v \frac{\partial u}{\partial r}=-\frac{\partial p}{\partial z}+\frac{1}{\operatorname{Re}}\left[\frac{\partial^{2} u}{\partial r^{2}}+\frac{1}{r} \frac{\partial u}{\partial r}\right] \\
\frac{\partial v}{\partial t}+u \frac{\partial v}{\partial z}+v \frac{\partial v}{\partial r}=-\frac{\partial p}{\partial r}+\frac{1}{\operatorname{Re}}\left[\frac{\partial^{2} v}{\partial r^{2}}+\frac{1}{r} \frac{\partial v}{\partial r}-\frac{v}{r^{2}}\right] \\
\frac{\partial u}{\partial z}+\frac{\partial v}{\partial r}+\frac{v}{r}=0 .
\end{gathered}
$$

The corresponding Reynolds number $\operatorname{Re}=D \rho u_{a v} / \mu$ equals 1 . If $u_{i n}=u_{a v}=1 / 24$, then according to (4.65) the pressure gradient is $\mathrm{dp} / \mathrm{d} z=-1 / 3$, and the velocity profile approaches the parabolic profile of steady Hagen-Poiseuille flow, $u(r)=(1-$ $\left.r^{2}\right) / 12$. The resulting velocity field at dimensionless time $t=1.0$ is shown in Figure 4.4. The development of the steady state parabolic profile is clearly evident. The radial component of the velocity deviates from zero only in the entrance region. The maximum norm of the error in the axial velocity for different grid sizes is given in Table 4.1. We have used the first-order method described in section4.2 and Table 4.1 confirms the first-order convergence of the numerical solution.

\begin{tabular}{cccc}
\hline $\mathrm{N}_{z} \times \mathrm{N}_{\mathrm{r}}$ & $\mathrm{N}_{\mathrm{t}}$ & $\mathrm{h}_{1}, \mathrm{~h}_{2}, \tau$ & error \\
\hline \hline $20 \times 20$ & 20 & $\mathrm{~h}_{1}=0.158, \mathrm{~h}_{2}=0.053, \tau=0.053$ & $4.17 \cdot 10^{-3}$ \\
$40 \times 40$ & 40 & $\mathrm{~h}_{1}=0.078, \mathrm{~h}_{2}=0.0256, \tau=0.0256$ & $2.0 \cdot 10^{-3}$ \\
$100 \times 100$ & 100 & $h_{1}=0.03, h_{2}=0.01, \tau=0.01$ & $8.4 \cdot 10^{-4}$ \\
\hline
\end{tabular}

Table 4.1: Error in the axial velocity at $z=\mathrm{L}$ for Hagen-Poiseuille flow. 


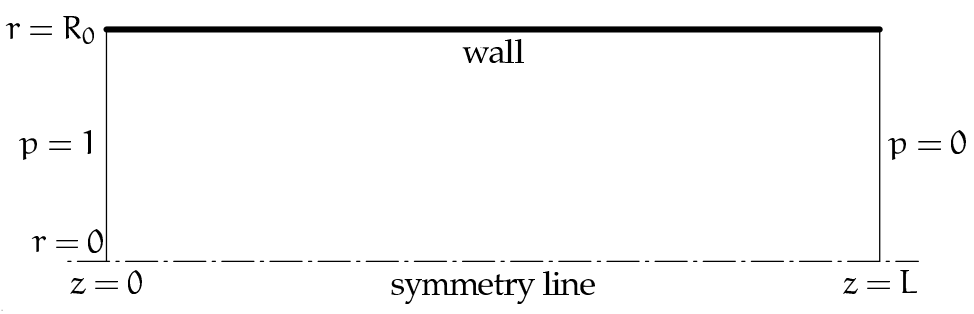

Figure 4.5: Configuration of the test problem: starting flow in a circular pipe.

\subsubsection{Starting flow in a circular pipe}

Another example with a known solution is starting laminar incompressible flow in a pipe. We take the same flow parameters as in the previous example. Suppose that the fluid is at rest at $t=0$, at which time a sudden, uniform and constant pressure gradient $\mathrm{dp} / \mathrm{d} z$ is applied. An axial flow will commence which gradually approaches the steady Hagen-Poiseuille flow. The analytical solution, derived by Szymanski in 1932 [68], gives the deviation of $u$ from the Hagen-Poiseuille flow at any time

$$
\frac{u(r, t)}{u_{\max }}=\left(1-r^{2}\right)-\sum_{n=1}^{\infty} \frac{8 J_{0}\left(\lambda_{n} r\right)}{\lambda_{n}^{3} J_{1}\left(\lambda_{n}\right)} \exp \left(-\lambda_{n}^{2} t\right)
$$

where $u_{\max }=-(d p / d z) R_{0}^{2} / 4 \mu$. Here $J_{0}$ and $J_{1}$ are Bessel functions of the first kind of zero and first order, respectively, and $\lambda_{n}$ are the roots of the Bessel function $J_{0}$.

Figure 4.5 illustrates the configuration of this test problem. The difference with the previous example in Section 4.3.1 is that we define constant pressures at the inflow and outflow boundaries of the domain. The initial conditions are zero flow and a constant pressure gradient. This example allows us not only to find the steady state solution, but also to see the development of the parabolic velocity profile in time.

The velocity profiles during flow acceleration for various values of the dimensionless time are plotted in Figure 4.6(a). They were obtained on $(40 \times 40)$-point regular grid and with time step $\tau=0.0256$. It is clear that the same results, as shown by White [86, Fig. 3-14], are reproduced with our code. The accuracy is assessed using the infinity norm of the difference between the analytical and numerical solution: $\|\epsilon\|_{\infty}=\left\|u_{a n}-u_{n u m}\right\|_{\infty}$. The error as function of time for different grids is also displayed in Figure 4.6(b). We start from the rest situation at $t=0$, where the error is zero. During the flow acceleration the numerical error grows and it decreases when the velocity profile tends asymptotically to the parabolic distribution of steady flow.

\subsubsection{Flow due to an oscillating pressure gradient}

In this example we consider laminar incompressible flow through a circular pipe under the influence of a harmonically oscillating pressure gradient. With the assumption that the flow is independent of the axial coordinate, the Navier-Stokes equation 


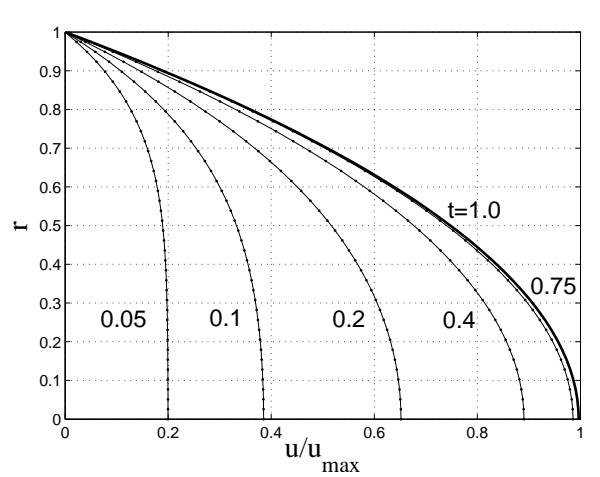

(a) velocity profiles at $z=\mathrm{L}$

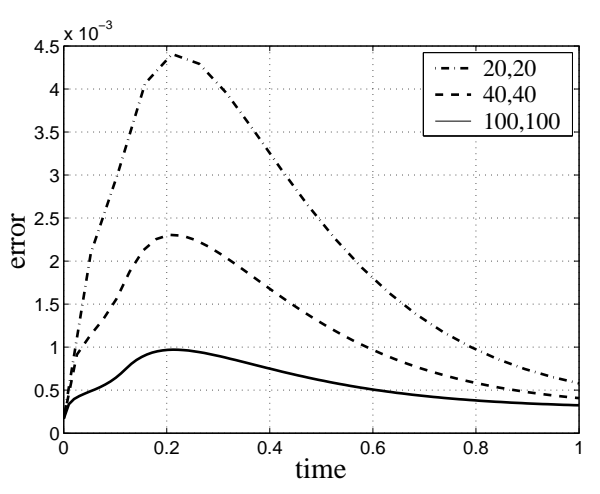

(b) error in velocity as function of time

Figure 4.6: Numerical results for starting flow in a pipe.

has the form

$$
\frac{\partial u}{\partial t}=-\frac{1}{\rho} \frac{\partial p}{\partial z}+v\left[\frac{\partial^{2} u}{\partial r^{2}}+\frac{1}{r} \frac{\partial u}{\partial r}\right] .
$$

We shall assume that the pressure gradient varies harmonically with time,

$$
\frac{\partial p}{\partial z}=-\rho K \cos (\omega t)
$$

where $\mathrm{K}$ is a constant. If we use complex notation and put

$$
\frac{\partial p}{\partial z}=-\rho K e^{i \omega t}
$$

then the analytical solution found by Grace (1928) [21] and Sexl (1930) [62] is given by

$$
u(r, t)=\frac{K}{i \omega} e^{i \omega t}\left[1-\frac{J_{0}(r \sqrt{-i \omega / v})}{J_{0}\left(R_{0} \sqrt{-i \omega / v}\right)}\right] .
$$

We introduce dimensionless variables, denoted by a hat, via

$$
t=\frac{1}{\omega} \widehat{t}, r=R_{0} \widehat{r}, u=\bar{u} \hat{u}, p=\bar{\rho} \bar{u}^{2} \hat{p} .
$$

The non-dimensional equation (4.78), the hats on the dimensionless variables are omitted, is

$$
\frac{\partial u}{\partial t}=-\frac{1}{\rho} \frac{\partial p}{\partial z}+\frac{1}{\operatorname{Re}_{\omega}}\left[\frac{\partial^{2} u}{\partial r^{2}}+\frac{1}{r} \frac{\partial u}{\partial r}\right] .
$$

The quantity $\operatorname{Re}_{\omega}=R_{0}^{2} \omega / v$ is called the kinetic Reynolds number and it is a measure for the viscous effects in oscillating flows, see section 2.3.1. Using series approximations for the Bessel functions, approximations for the velocity can be obtained, see [86, p. 144]. 


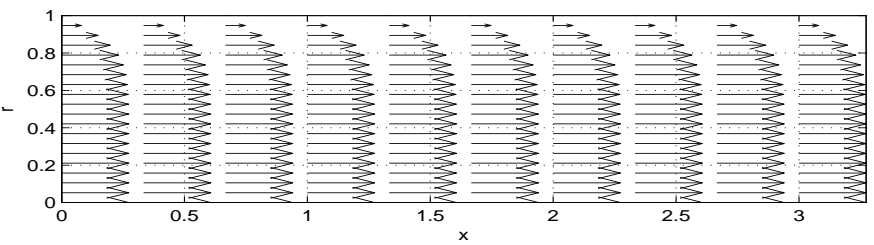

(a) $\omega t=\pi / 2$

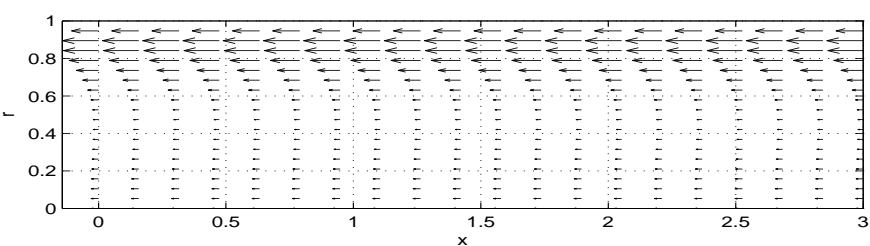

(b) $\omega t=\pi$

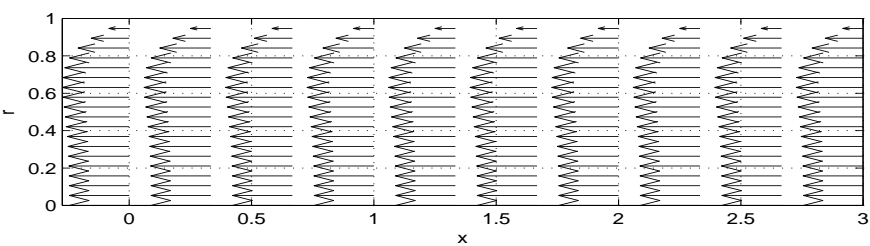

(c) $\omega t=3 \pi / 2$

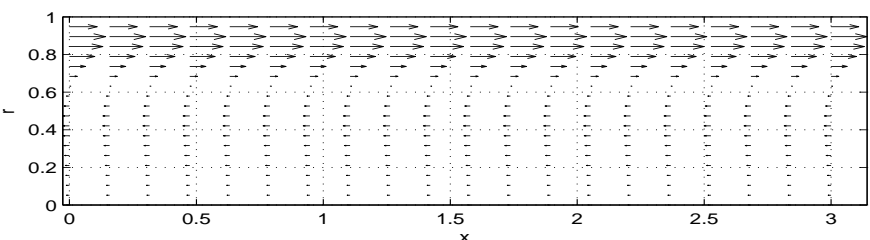

(d) $\omega t=2 \pi$

Figure 4.7: Velocity fields of the oscillating flow at different times in the pressure cycle, $\operatorname{Re}_{\omega}=100$. 


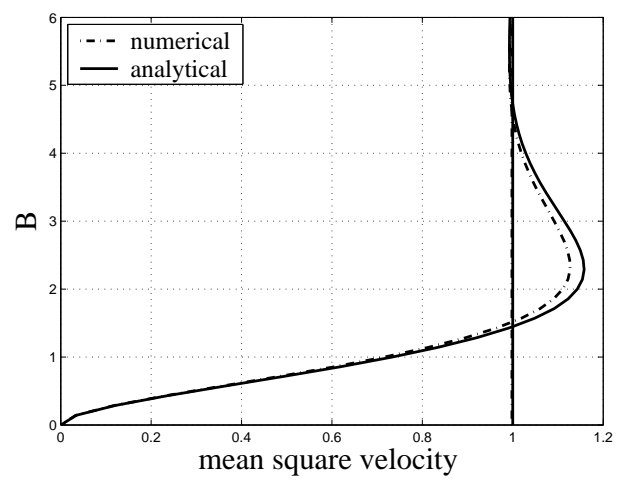

Figure 4.8: The mean square of the velocity distribution as a function of

$$
\mathrm{B}=\sqrt{\operatorname{Re}_{\omega}}(1-\widehat{r}), \operatorname{Re}_{\omega}=100 \text { (Richardson's annular effect). }
$$

For $\operatorname{Re}_{\omega}<4$ :

$$
\begin{gathered}
\frac{u(r, t)}{u_{\max }} \approx\left(1-\widehat{r}^{2}\right) \cos (\omega t)+\frac{\operatorname{Re}_{\omega}}{16}\left(\widehat{r}^{4}+4 \widehat{r}^{2}-5\right) \sin (\omega t)+\mathcal{O}\left(\operatorname{Re}_{\omega}^{2}\right), \\
u_{\max }=\frac{K R_{0}^{2}}{4 v} .
\end{gathered}
$$

Remembering that $d p / d z$ is proportional to $\cos (\omega t)$, we see that for very small $\operatorname{Re}_{\omega}$ the flow is nearly a quasi-steady Hagen-Poiseuille flow in phase with the slowly varying pressure gradient.

For $\operatorname{Re}_{\omega}>4$ :

$$
\begin{gathered}
\frac{u(r, t)}{u_{\max }} \approx \frac{4}{\operatorname{Re}_{\omega}}\left[\sin (\omega t)-\frac{e^{-B}}{\sqrt{\widehat{r}}} \sin (\omega t-B)\right]+\mathcal{O}\left(\operatorname{Re}_{\omega}^{-2}\right), \\
B=(1-\widehat{r}) \sqrt{\frac{\operatorname{Re}_{\omega}}{2}} .
\end{gathered}
$$

At large $\operatorname{Re}_{\omega}$ the flow approximately lags the pressure gradient by $\pi / 2$. The second term in expression (4.83) quickly damps out as the distance from the wall $1-\widehat{r}$ becomes larger. Consequently at a large distance from the wall only the first term is important, which is independent of that distance.

For the example problem with parameter values $L=3[\mathrm{~m}], R_{0}=1[\mathrm{~m}], v=$ $1\left[\mathrm{~m}^{2} \cdot \mathrm{s}^{-1}\right], \mathrm{K}=10\left[\mathrm{~m} \cdot \mathrm{s}^{-2}\right], \omega=100\left[\mathrm{~s}^{-1}\right]$, the kinetic Reynolds number is $\operatorname{Re}_{\omega}=100$. The results of the numerical simulation at different times in the pressure cycle are presented in Figure 4.7.

As can be seen, at times $\omega t=\pi$ and $2 \pi$ there is a region of high-velocity flow near the wall. This effect is characteristic for flow oscillating at high frequencies and was 


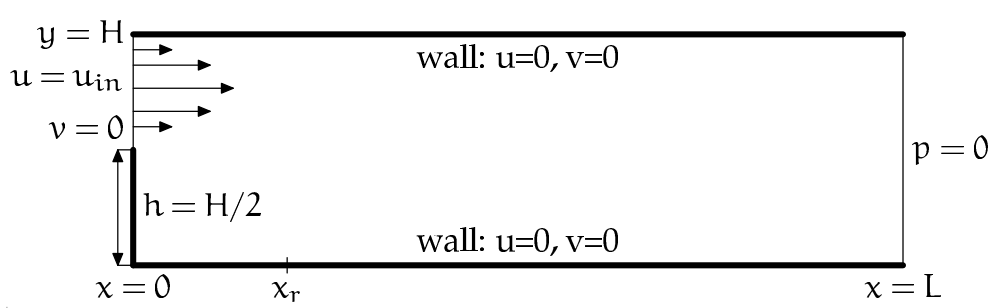

Figure 4.9: Configuration of the test problem: flow over a backward-facing step.

first observed by Richardson and Tyler in 1929 [58]. The overshoot in velocity, now called Richardson's annular effect, was predicted theoretically by Witzig in 1914 [89], Grace in 1928 [21] and Sexl in 1930 [62]. By averaging equation (4.83) over one cycle, we obtain an analytical expression for the mean square velocity

$$
\overline{u^{2}(r)}=\frac{K^{2}}{2 \omega^{2}}\left[1-\frac{2 e^{-B}}{\sqrt{r}} \cos (B)+\frac{e^{-2 B}}{r}\right] .
$$

The maximum in the mean velocity occurs for $B \approx 2.284$, where $B$ is the dimensionless distance from the wall, weighted by $\sqrt{\operatorname{Re}_{\omega} / 2}$. The numerical result for the mean square velocity and the analytical curve are given in Figure 4.8. The computation is performed with a $(20 \times 100)$-point grid for two pressure cycles with 100 time steps per cycle. The numerical and analytical predictions of the overshoot location are the same.

\subsubsection{Flow over a backward-facing step}

Our next example is the classical problem of laminar incompressible flow over a backward-facing step. There have been many numerical and experimental studies investigating this type of flow, see [1], [5], [19], [22]. This is one of the simplest geometrical cases that provides an interesting, non-trivial flow. The main feature of the flow is the presence of recirculation zones, one on the lower wall and one on the upper wall. These recirculating regions and vortex-shedding phenomena, encountered in most flows of practical engineering interest, make the backward-facing step both a relevant and severe test case for our code.

The dimensionless parameters for the computational domain are: length $L=20 \mathrm{H}$, height $H=1$, step height $h=H / 2$. Figure 4.9 displays the flow geometry and boundary conditions. We are interested in the time-dependent simulation of a flow that starts instantaneously. The problem is described by the Navier-Stokes equations for unsteady incompressible viscous flow

$$
\begin{aligned}
& \frac{\partial u}{\partial t}+u \frac{\partial u}{\partial x}+v \frac{\partial u}{\partial y}=-\frac{\partial p}{\partial x}+\frac{1}{\operatorname{Re}}\left(\frac{\partial^{2} u}{\partial x^{2}}+\frac{\partial^{2} u}{\partial y^{2}}\right), \\
& \frac{\partial v}{\partial t}+u \frac{\partial v}{\partial x}+v \frac{\partial v}{\partial y}=-\frac{\partial p}{\partial y}+\frac{1}{\operatorname{Re}}\left(\frac{\partial^{2} v}{\partial x^{2}}+\frac{\partial^{2} v}{\partial y^{2}}\right),
\end{aligned}
$$




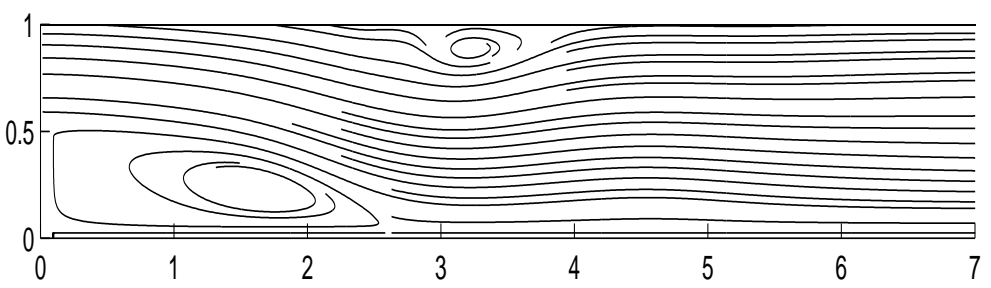

(a) $\mathrm{t}=5$

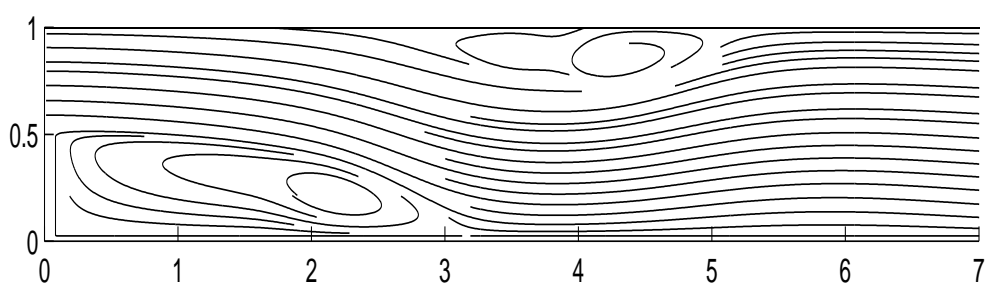

(b) $t=10$

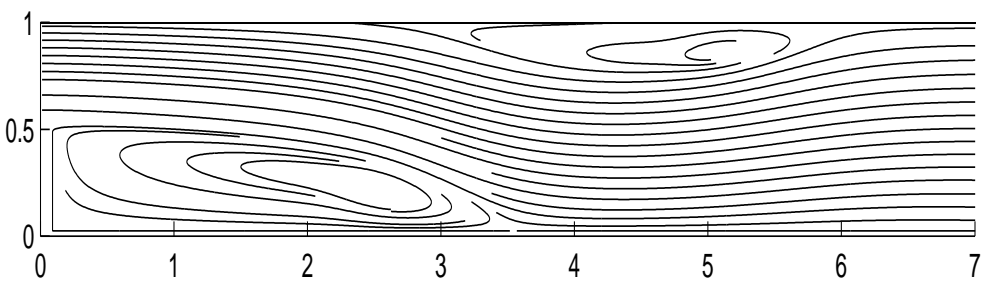

(c) $\mathrm{t}=15$

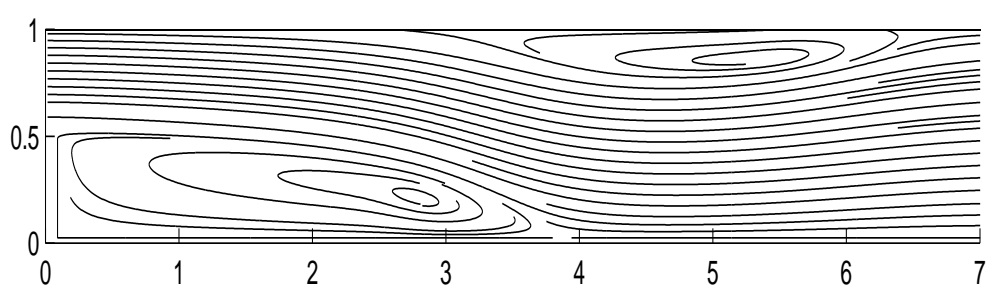

(d) $t=20$

Figure 4.10: Development in time of the streamlines of flow over a backward-facing step at $\operatorname{Re}=800$ between $x=0$ and $x=7$. 


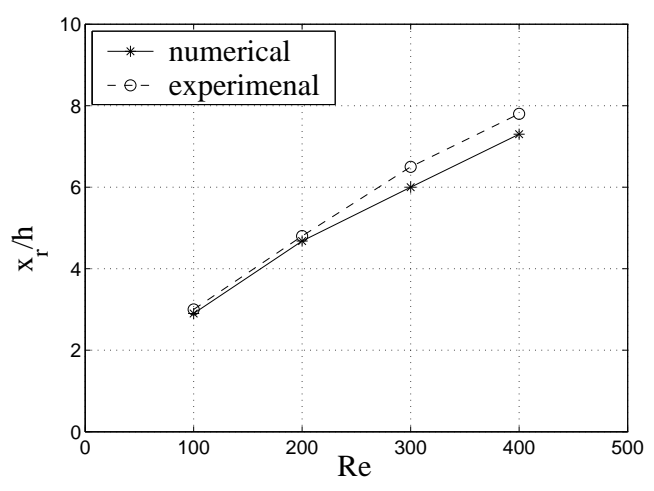

Figure 4.11: Reattachment length versus Reynolds number.

$$
\frac{\partial u}{\partial x}+\frac{\partial v}{\partial y}=0 .
$$

At the inlet, the velocity has a parabolic profile:

$$
u_{i n}=u(y)=\left\{\begin{array}{rll}
24(y-1)(0.5-y) & \text { if } & H / 2 \leq y \leq H, \\
0 & \text { if } & 0<y<H / 2 .
\end{array}\right.
$$

The computations have been performed on a $(200 \times 40)$-point regular grid with a time step $\tau=0.0025$. The transient evolution of flow at $\operatorname{Re}=800$ is presented in Figure 4.10 as a sequence of streamline plots for different values of the dimensionless time $t=5,10,15$ and 20. In Figure 4.10(a) at $t=5$, the lower and upper eddies are already present, although both eddies are far upstream from their final positions. Further in time they are slowly creeping down the walls and stretching. Far downstream the flow develops to the parabolic Poiseuille regime. The portrayal of the transient evolution, given in Figure 4.10, is qualitatively in good agreement with the time history plots of the streamwise velocity component given by Gresho et al. [22].

The accuracy of our numerical method can be assessed if we study the dependence of the reattachment length $x_{r}$, i.e. the horizontal distance between the step and the point where the recirculation zone ends, on the Reynolds number. The numerical results for $\operatorname{Re}=100,200,300,400$, shown in Figure 4.11, are close to the experimental results of Armaly et al. [1]. For larger values of Re, computational results start to deviate from the experimental results. The difference between the experimental results and the numerical results is usually attributed to the fact that the flow becomes three-dimensional for values of Re larger than 400 , thus questioning the existence of a two-dimensional steady state.

\subsubsection{Temperature distribution in fully developed pipe flow}

In the previous sections we validated our numerical method from the point of view of velocity computation. The flow was incompressible and isothermal. We also need 
tests for the temperature computation. The following two problems with analytical solutions are used

- temperature distribution for fully developed flow in a circular pipe with constant heat flux at the wall,

- temperature distribution for fully developed flow in a circular pipe with constant wall temperature.

Because of early studies by Graetz in 1883, the problems under consideration are usually called "Graetz problems" [60]. We consider an incompressible fluid with constant physical properties, having a fully developed Hagen-Poiseuille velocity profile $u(r)$. For hydrodynamically fully developed flow the heat transfer between fluid and wall can be studied theoretically. When the velocity profile is not influenced by heat transfer, the energy equation can be solved analytically and the fully developed temperature profile will be found.

The dimensionless unsteady energy balance in a circular tube gives

$$
\frac{\partial T}{\partial t}+u \frac{\partial T}{\partial z}+v \frac{\partial T}{\partial r}=\alpha\left(\frac{1}{r} \frac{\partial T}{\partial r}+\frac{\partial^{2} T}{\partial r^{2}}+\frac{\partial^{2} T}{\partial z^{2}}\right) \text { with } \alpha=\frac{k_{g}}{\rho c_{p}} .
$$

Assuming that there is no convective transport in the radial direction and no conduction in axial direction, the steady energy equation becomes

$$
\begin{gathered}
\frac{\alpha}{r} \frac{\partial}{\partial r}\left(r \frac{\partial T}{\partial r}\right)=u \frac{\partial T}{\partial z} \\
u(r)=2 u_{a v}\left(1-\frac{r^{2}}{R_{0}^{2}}\right), u_{a v}-\text { mean velocity. }
\end{gathered}
$$

For the case of constant wall heat flux, equation (4.91) must be solved with the boundary conditions

$$
\begin{gathered}
\text { inflow }(z=0): \quad T=T_{0}=\text { constant, } \\
\text { wall }\left(r=R_{0}\right): \quad-k_{g} \frac{\partial T}{\partial r}=q_{w}=\text { constant, } \\
\text { symmetry line }(r=0): \quad \frac{\partial T}{\partial r}=0 .
\end{gathered}
$$

For the fully developed thermal field a constant heat flux results in

$$
\frac{\partial \mathrm{T}}{\partial z}=\frac{\partial \mathrm{T}_{w}}{\partial z}=\text { constant }
$$

where $T_{w}$ is the temperature of the wall. It means that the axial temperature gradient is independent of $r$. Integrating equation (4.91) two times and substituting the boundary conditions, we obtain

$$
\mathrm{T}(z, \mathrm{r})=\mathrm{T}_{w}(z)-\frac{\mathrm{u}_{\mathrm{a} v} \mathrm{R}_{0}^{2}}{8 \alpha} \frac{\partial \mathrm{T}}{\partial z}\left(3-\frac{4 \mathrm{r}^{2}}{\mathrm{R}_{0}^{2}}+\frac{\mathrm{r}^{4}}{\mathrm{R}_{0}^{4}}\right) .
$$


For the case of constant wall temperature, equation (4.91) must be solved with the boundary conditions (4.93), (4.95) and

$$
\text { wall }\left(\mathrm{r}=\mathrm{R}_{0}\right): \quad \mathrm{T}=\mathrm{T}_{w}=\text { constant. }
$$

The steady state series solution of equation (4.91) with boundary conditions (4.93)(4.94) was first found by Graetz in 1885 and rediscovered by Nusselt in 1910. The first four terms in the series are

$\mathrm{T}(z, \mathrm{r})=1-1.477 \mathrm{e}^{-3.658 z / \mathrm{Pe}} \mathrm{R}_{0}(\mathrm{r})-0.810 \mathrm{e}^{-22.178 z / \mathrm{Pe}} \mathrm{R}_{1}(\mathrm{r})+0.385 \mathrm{e}^{-53.05 z / \mathrm{Pe}} \mathrm{R}_{2}(\mathrm{r})+\ldots$,

where $R_{0}(r), R_{1}(r), R_{2}(r)$ are tabulated by Jakob [34, p. 455]. For $r=0: R_{0}(0)=$ $R_{1}(0)=R_{2}(0)=\ldots=1$, so that $T(z, 0)$ is directly available from equation (4.98).

For testing our numerical method, the following non-dimensional unsteady problem was solved

$$
\frac{\partial T}{\partial t}=-u \frac{\partial T}{\partial z}+\frac{1}{P e}\left(\frac{1}{r} \frac{\partial T}{\partial r}+\frac{\partial^{2} T}{\partial r^{2}}\right) \text { with } u(r)=2 u_{a v}\left(1-\frac{r^{2}}{R_{0}^{2}}\right) .
$$

The dimensionless parameters of the test problem are: length of the tube $\mathrm{L}=30$, radius $R_{0}=1$, average velocity $u_{a v}=1$ and Peclet number $P e=u_{a v} R_{0} / \alpha=60$. The boundary and initial conditions are defined as follows

$$
\begin{gathered}
\operatorname{inflow}(z=0): T(0, r, t)=0 \\
\text { wall }\left(r=R_{0}\right):\left\{\begin{array}{l}
\frac{\partial T}{\partial r}\left(z, R_{0}, t\right)=1 \text { constant wall flux } \\
\text { or } \\
T\left(z, R_{0}, t\right)=1 \quad \text { constant wall temperature }
\end{array}\right. \\
\text { symmetry line }(r=0): \quad \frac{\partial T}{\partial r}(z, 0, t)=0 \\
\text { initial conditions }(t=0): \quad T(z, r, 0)=0 .
\end{gathered}
$$

The steady state numerical solutions, obtained with a $(100 \times 20)$-point grid and with time step $\tau=0.05$, are presented in Figure 4.12. The steady state is reached at non-dimensional time $t=60$. To validate our steady state numerical results, a comparison with the two analytical solutions is made. For the case of constant wall flux, the radial temperature profiles of the numerical solutions at $t=10,20,30,60$ together with the analytical solution, given by (4.96), are plotted in Figure 4.13. For constant wall temperature, the axial temperature profiles of the numerical solutions at $t=10,15,20,60$ and the analytical solution (4.98) are plotted in Figure 4.14. The agreement is good, except for the points near the inflow and, to a less extent, outflow boundaries. The analytical series solution for the case of constant wall temperature is inaccurate near $z=0$. The boundary condition (4.100) specifies $T(0,0)=0$, but (4.98) gives $T(0,0)=-0.052$. This means that more than four terms are required to closely satisfy the specified boundary condition. 


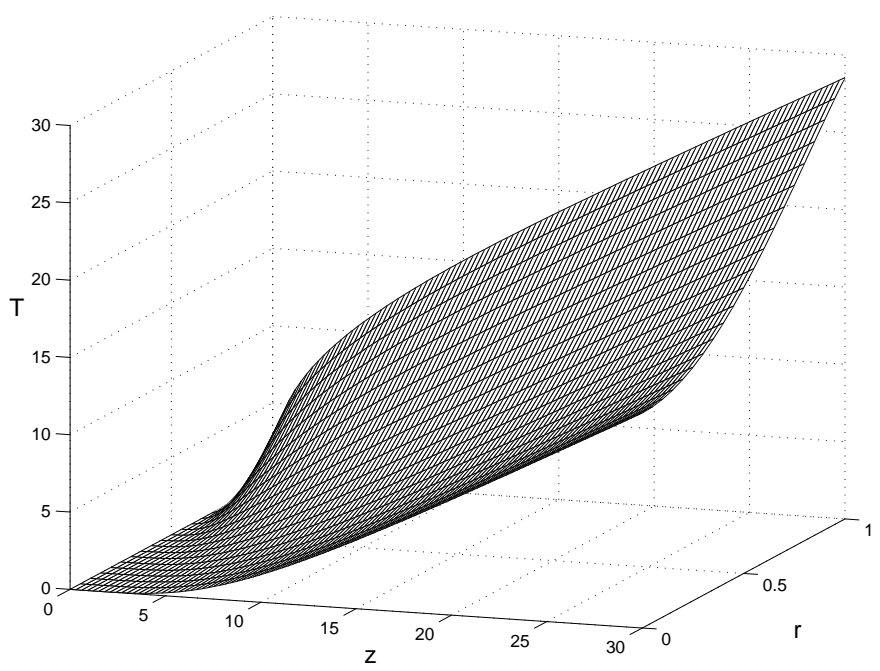

(a) constant wall flux

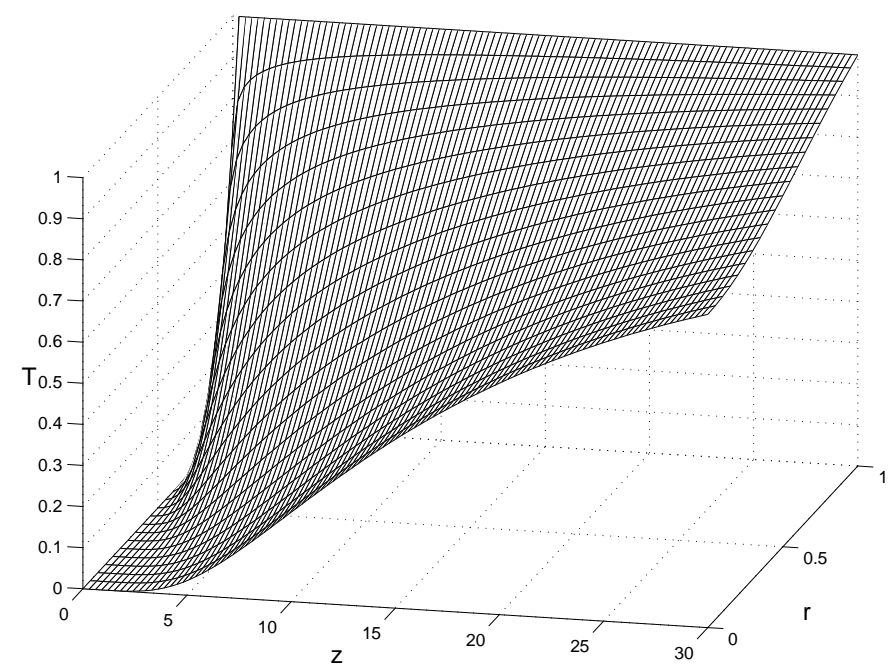

(b) constant wall temperature

Figure 4.12: Steady state numerical solutions $\mathrm{T}(z, \mathrm{r})$ for the Graetz problems. 


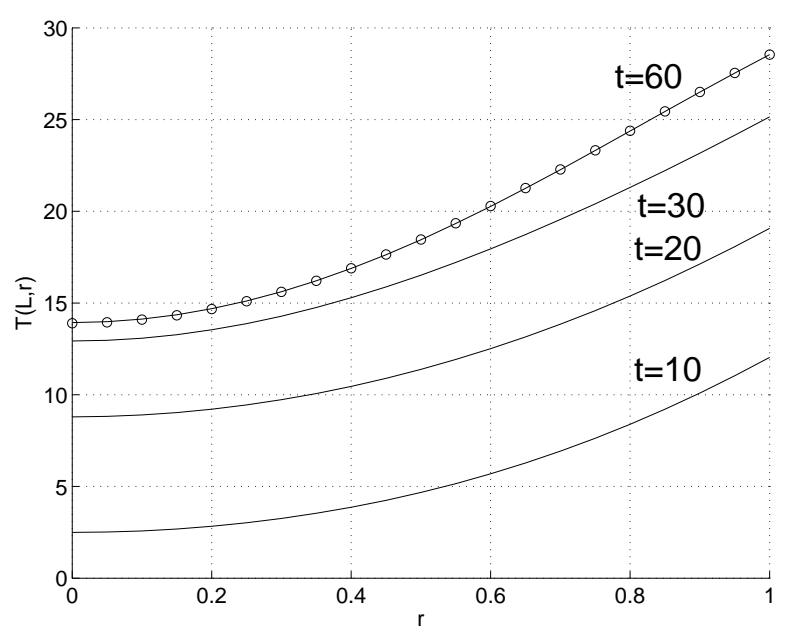

Figure 4.13: Graetz problem with constant heat flux at the wall. Radial temperature profiles of the numerical solutions at different times and analytical steady state solution (4.96) (dots).

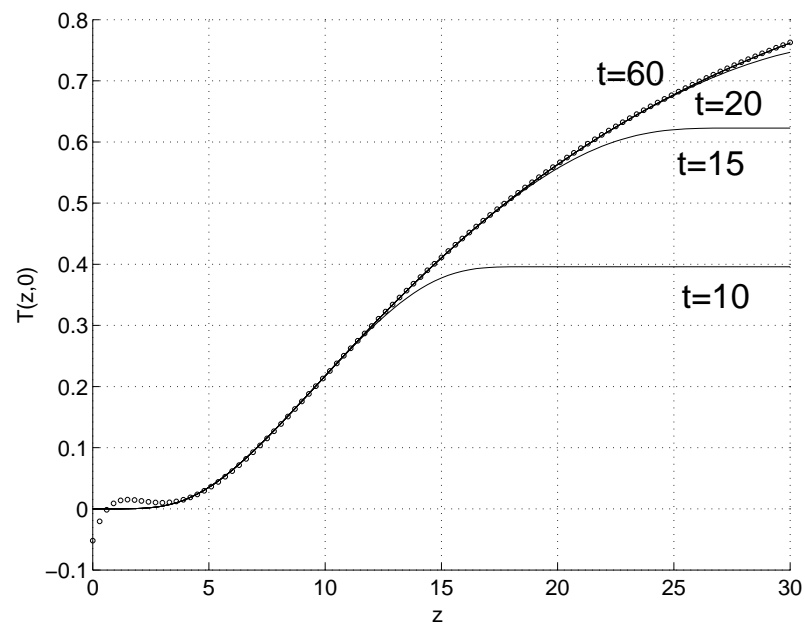

Figure 4.14: Graetz problem with constant wall temperature. Axial temperature profiles of the numerical solutions at different times and analytical steady state solution (4.98) (dots). 


\section{Flow and heat transfer computations for the pulse tube}

In the previous chapters we derived one-dimensional and two-dimensional mathematical models and we analysed numerical techniques needed for the simulation of heat transfer in oscillating compressible laminar flow. The described methods have been implemented in a newly developed simulation tool. In this chapter computations for a typical pulse-tube refrigerator are presented. First, the one-dimensional results are discussed. The velocity, temperature, mass flow and enthalpy flow are investigated for two driving pressures: sinusoidal and trapezoidal. The developed model is validated by comparing the results for the sinusoidal driving pressure with a first-order harmonic analysis. The one-dimensional results have been obtained at low computational cost and they serve as a reference for the two-dimensional results, presented secondly. In the two-dimensional model radial thermal and viscous effects are taken into account. The heat transfer between gas and wall is studied in detail.

\subsection{One-dimensional results}

The physical parameters and corresponding non-dimensional numbers used in our simulations are given in Appendix A. These parameter values are representative of a large scale, high-frequency orifice pulse tube. In our numerical simulations the global pressure, $P(t)$, is of sinusoidal or trapezoidal shape, as shown in Figure 5.1. In practice, any realistic pressure, for example measured data, can be accommodated by our model. Results of this section are obtained using the equations (3.1), (3.2), together with the boundary conditions (3.6), (3.7), (3.8) and initial conditions (3.9), (3.10). 


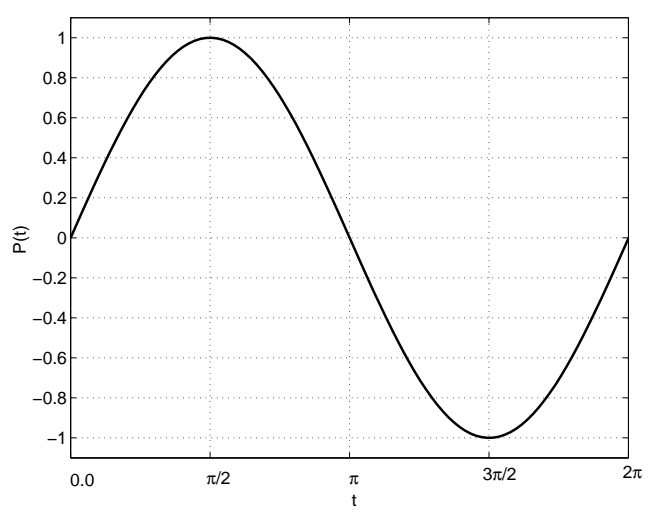

(a) sinusoidal driving pressure

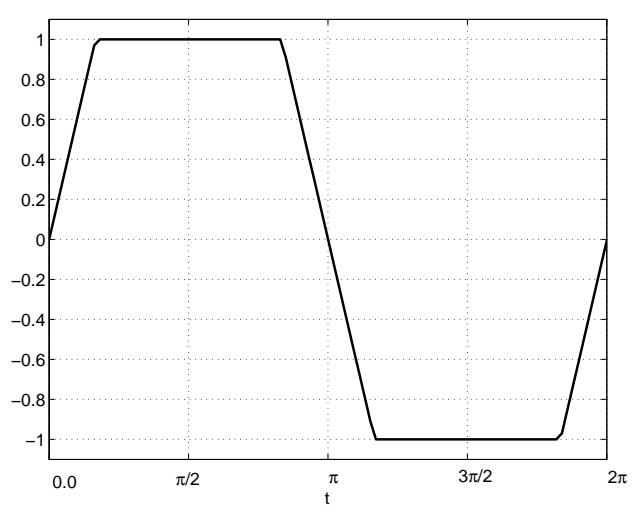

(b) trapezoidal driving pressure

Figure 5.1: Dimensionless driving pressures used in the simulations.

\subsubsection{Velocity}

For the velocity computation we have used equation (3.1) and boundary condition (3.6). The buffer pressure can be considered either as constant or it can be obtained from equation (2.52). The difference is noticeable only during the first cycles of the simulation, when the oscillations are not steady yet.

In Figure 5.2 velocities for four different times in the third pressure cycle are presented. The dimensionless period of the pressure variations is $t_{c}=2 \pi$. For a pulse tube working at $20 \mathrm{~Hz}$ frequency this corresponds to a dimensional time $t_{c}=0.05[\mathrm{~s}]$. At every instant the velocity is approximately a linear function of position. When the pressure has its maximum (at $t=4.5 \pi$ ) or minimum (at $t=5.5 \pi$ ), the velocities are constant for both the sinusoidal and trapezoidal pressure variations. At these times the pressure is equal to 1 or -1 and the pressure time derivatives are equal to zero. This means that the function $s_{1}(t)$ in equation (3.1) is zero. The resulting formula for the velocity is

$$
\frac{\partial u}{\partial x}=\epsilon \frac{\partial^{2} \mathrm{~T}}{\partial x^{2}} \text { with } \epsilon=\frac{1}{\mathcal{B}(\mathcal{A}+1)} \frac{1}{\mathrm{Pe}} \approx 3 \cdot 10^{-4} .
$$

As can be seen from the graphs at $t=4 \pi$ and $t=5 \pi$ faster pressure changes result in higher velocities. This follows directly from equation (3.1).

Figure 5.3 shows the velocities at the cold end and at the hot end as function of time for two different pressures. For the sinusoidal pressure variation the phase shift between the velocities at the cold and hot ends is approximately $\pi / 4$.

\subsubsection{Temperature dynamics}

Our model allows us to study the temperature dynamics in the tube. The results in Figure 5.4 have been obtained with the numerical schemes (3.12), (3.13) with Van 


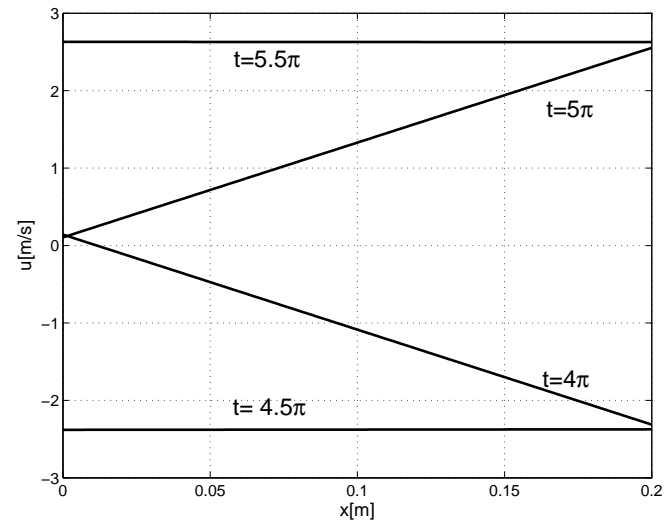

(a) sinusoidal driving pressure

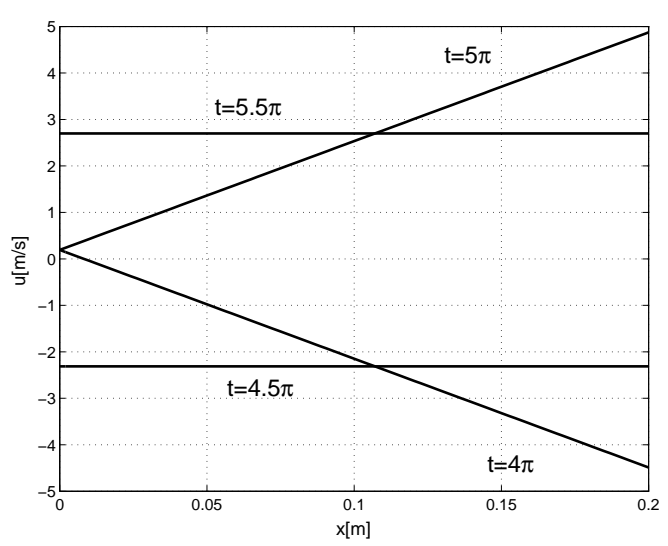

(b) trapezoidal driving pressure

Figure 5.2: Velocities for four different times in the third pressure cycle.

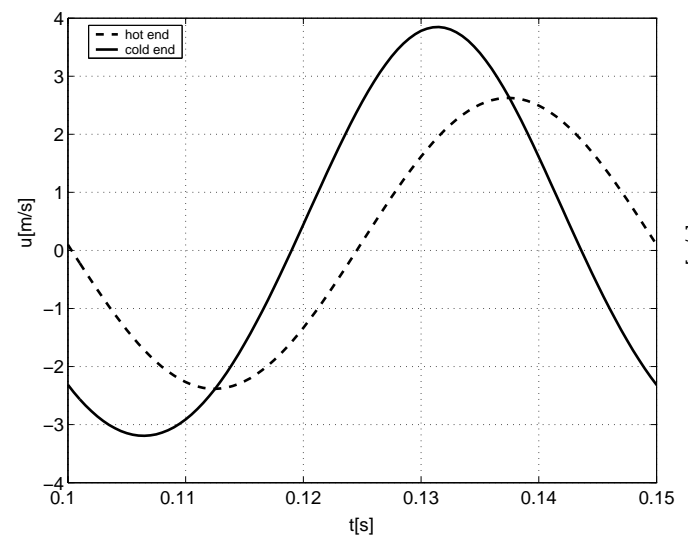

(a) sinusoidal driving pressure

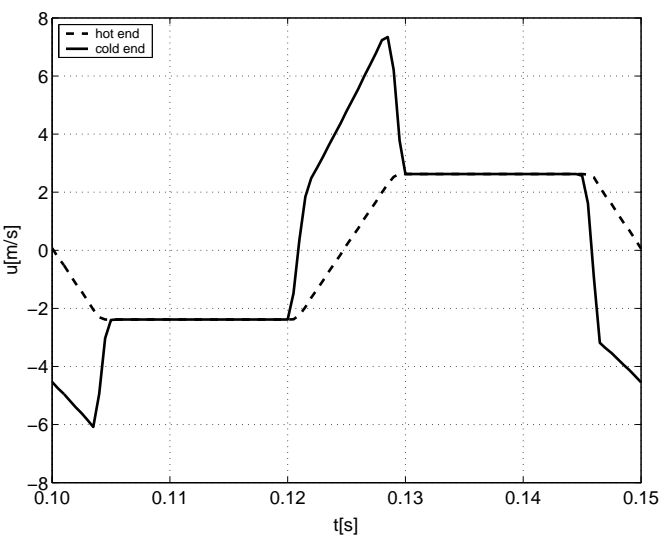

(b) trapezoidal driving pressure

Figure 5.3: Velocities at the cold and hot ends for sinusoidal and trapezoidal driving pressures during the third cycle. 
Leer flux limiter and with $h=0.015$. A variable time step was chosen according to condition (3.17). The resulting system of discretised equations has been solved using the iterative method BICGSTAB [59], with incomplete LU-factorisation as preconditioner. In Figure 5.4 calculated temperature distributions are depictured at times $\mathrm{t}=4 \pi, 4.5 \pi, 5 \pi$ and $5.5 \pi$, which correspond to different parts of the sinusoidal pressure cycle: $4 \pi$ - pressure increases, $4.5 \pi$ - maximum pressure, $5 \pi$ - pressure decreases, $5.5 \pi-$ minimum pressure. The heat exchanger temperatures at the tube ends are $\mathrm{T}_{\mathrm{H}}=300 \mathrm{~K}$ and $\mathrm{T}_{\mathrm{C}}=70 \mathrm{~K}$; a linear temperature profile has been taken as initial condition. The penetration of cold and hot gas at opposite sides of the tube is clearly seen. Part of the gas, called the gas piston, maintains a linear temperature profile during the entire simulation. This part of the gas undergoes adiabatic compression and expansion but never leaves the tube. There are large temperature gradients (contact discontinuities) in the tube between the gas piston and the boundary regions. Figure 5.5 shows temperature profiles on successively refined grids to show the convergence of our numerical solution. For comparison purposes we also plotted the solution without flux-limiter, obtained on the finest grid $(h=0.015)$.

The time integration proceeds until the system exhibits periodic behaviour. We require that the difference in temperature between two consecutive cycles for all discretised points to be less than a predefined tolerance. Concerning the expected periodicity of the solution, for $\mathrm{h}=0.015$ and linear initial temperature distribution, we need 9 cycles to achieve a situation where the maximum norm of the difference in temperature between two consecutive cycles for all discretised points is less than $10^{-4}$. For different initial conditions, for example a step-function initial temperature as plotted in Figure 5.6(b), many more cycles are needed to reach periodicity. The heat conduction of helium is very small, the thermal penetration depth, see [67],

$$
\delta_{k}=\sqrt{\frac{2 k_{g}}{\omega \rho c_{p}}}
$$

is approximately $2.6 \cdot 10^{-4}[\mathrm{~m}]$. This means that a time, proportional to $\delta_{\mathrm{k}}^{2}$, or $\mathrm{t} \sim$ $6.8 \cdot 10^{8}[\mathrm{~s}]$ is needed for the real system to come to a steady situation. It means $10^{10}$ cycles of 0.05 [sec]. In fact, on a coarse grid the numerical thermal penetration depth is larger than given by equation (5.2) and the resulting time to reach the steady situation is much shorter. A coarse mesh computation quickly finds an approximation to the steady state which then can be used as an initial condition for computations with locally refined grids. Figure 5.6 shows that the same steady solution is obtained with different initial conditions $(h=0.15)$.

The temperatures at the cold end and at the hot end for 20 cycles are displayed in Figure 5.7. In this picture the periodicity of the solution is clearly seen. Figure 5.8 shows the temperature near the cold and hot ends for one cycle. For the sinusoidal driving pressure, we can compare our results with the harmonic model described in [78] and with three-dimensional CFD results from [88]. The temperatures obtained with these two other models are also depictured in Figure 5.8. The results of the three models are consistent.

The one-dimensional and three-dimensional numerical results differ slightly from 


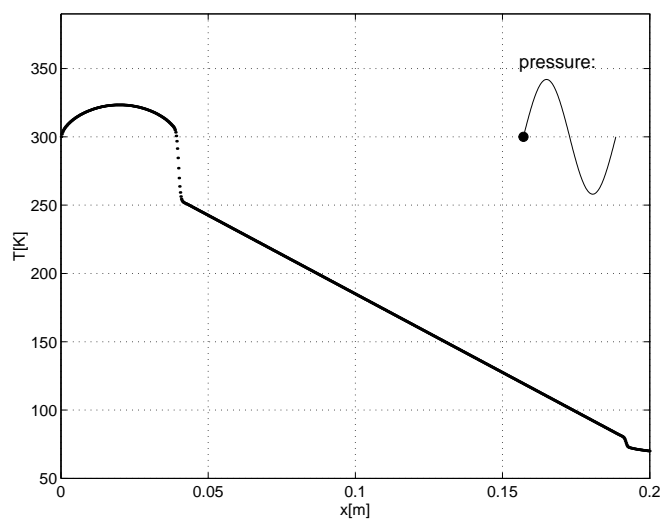

(a) $\mathrm{t}=4 \pi$

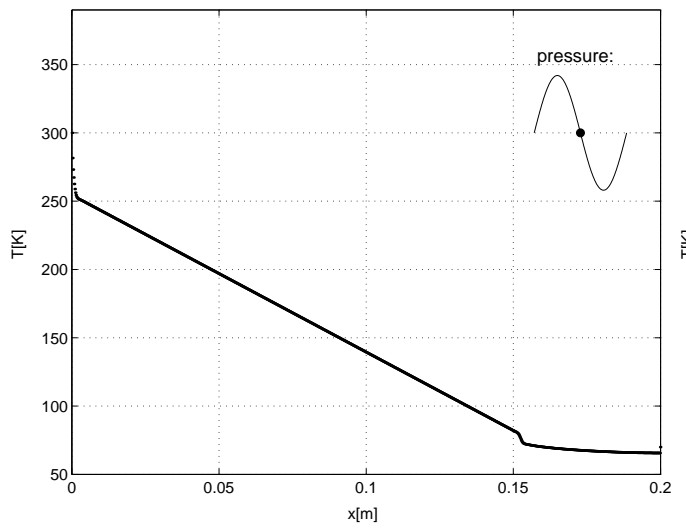

(c) $t=5 \pi$

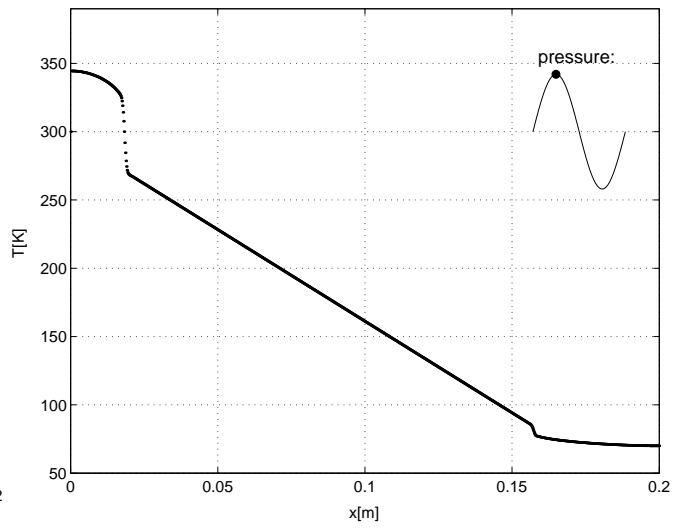

(b) $\mathrm{t}=4.5 \pi$

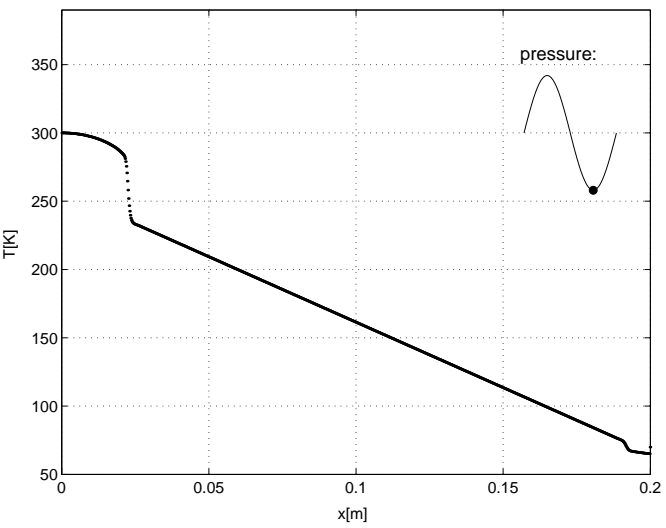

(d) $t=5.5 \pi$

Figure 5.4: Temperature for four different times in the third pressure cycle. 

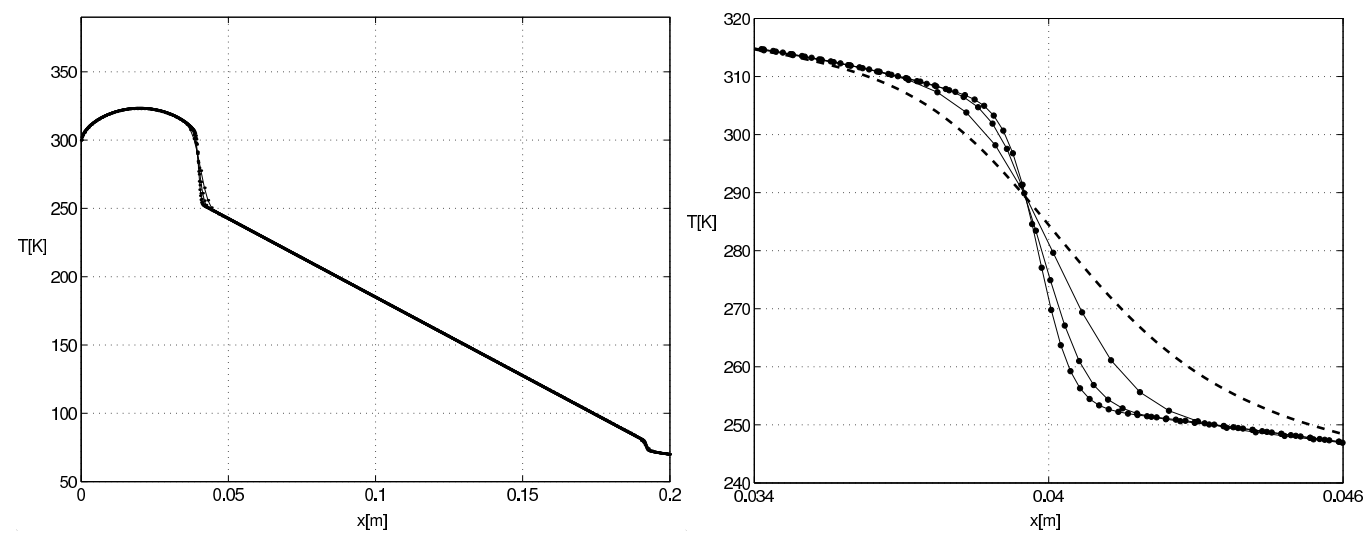

Figure 5.5: Temperature profiles at $t=4 \pi$ obtained on successively refined grids $(\mathrm{h}=$ $0.05,0.025,0.015)$. Dashed line: solution on the finest grid $(h=0.015)$ obtained without flux-limiter.

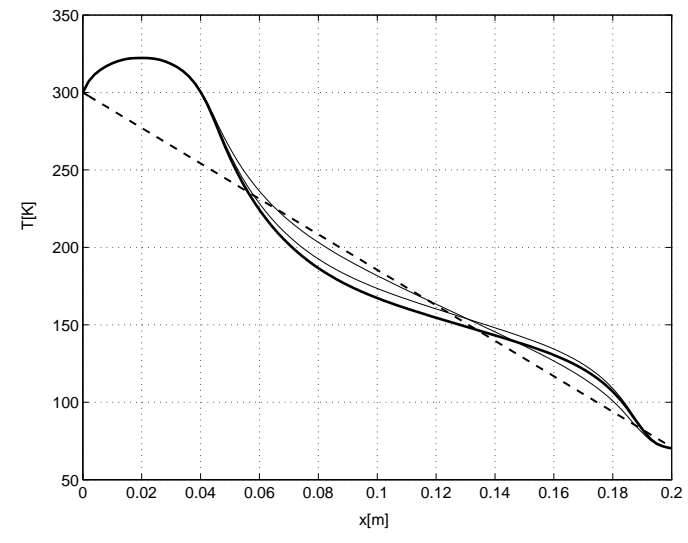

(a) linear initial temperature

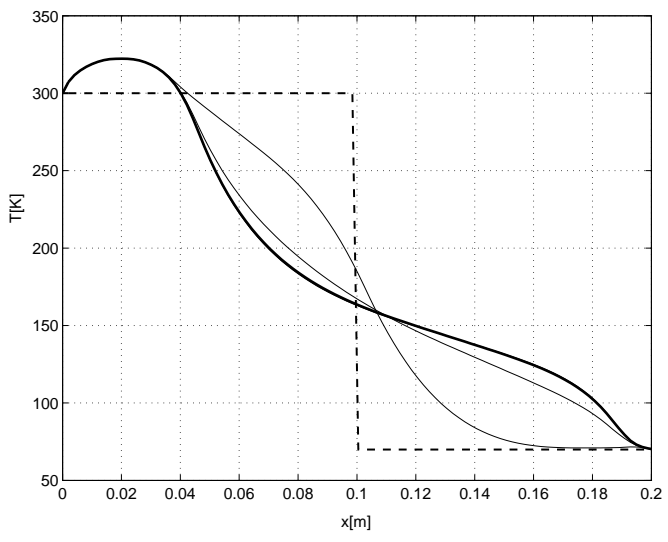

(b) step-function initial temperature

Figure 5.6: Temperature after 100, 500 (thin lines) and 1000 cycles (bold line), $\mathrm{t}=$ $200 \pi, 1000 \pi, 2000 \pi$, for two different initial conditions (broken lines). 


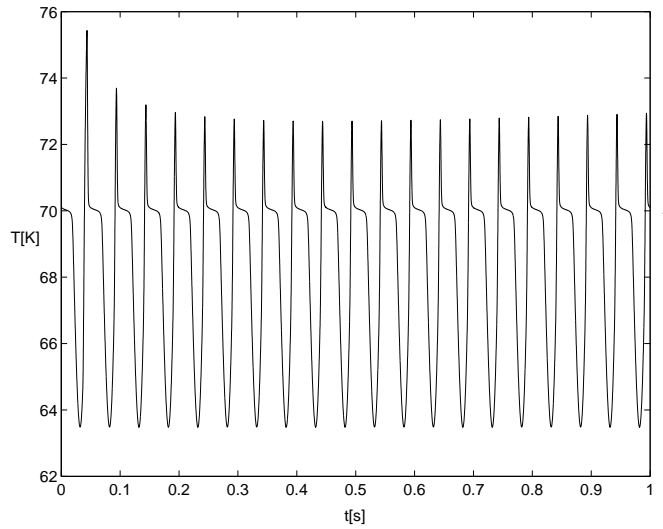

(a) cold end

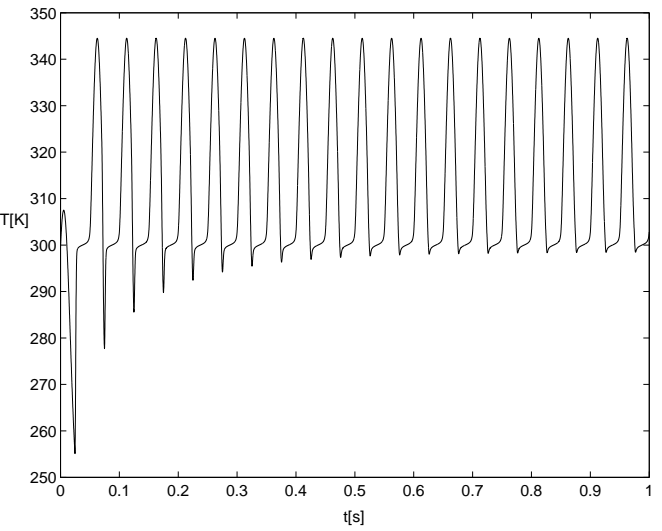

(b) hot end

Figure 5.7: Temperature at the cold end and the hot end for the first 20 cycles.

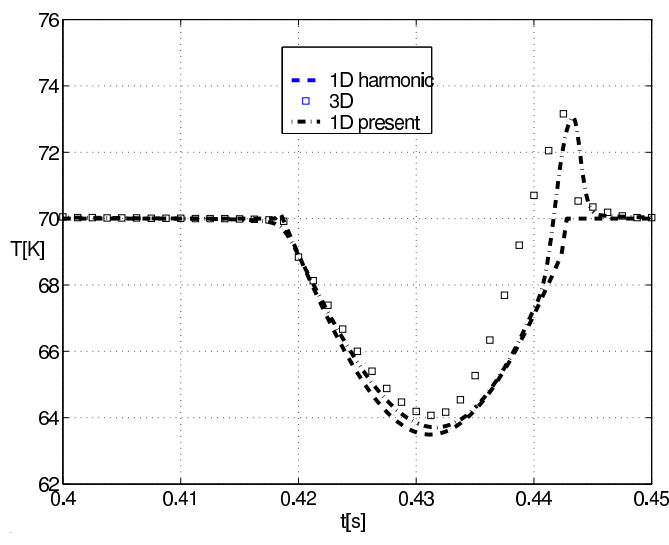

(a) cold end

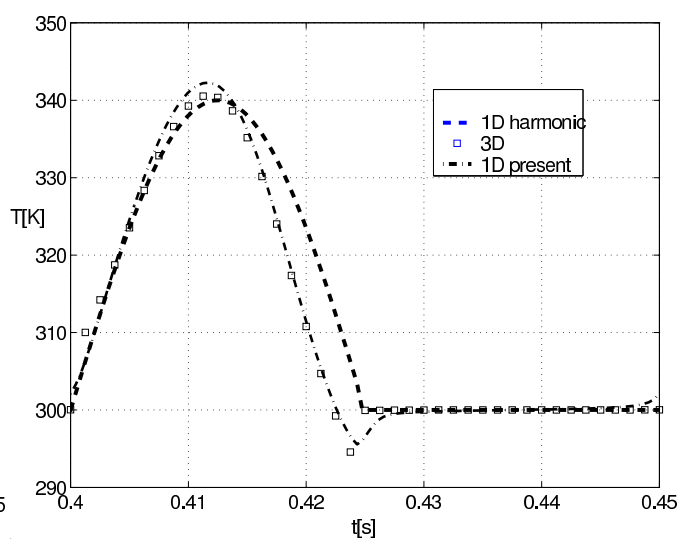

(b) hot end

Figure 5.8: Temperature at the cold and hot end after 9 cycles for $h=0.015$ (sinusoidal pressure variation). 
the harmonic results: they give an overshoot in temperature at the cold and hot end boundaries. This overshoot is also present in other one-dimensional simulations of single-inlet pulse tubes, see [35], [82], [84]. The same effect was reported in [83] for a double-inlet pulse tube. Is this effect real or is it just an artifact of the simulation model? A possible physical explanation is the presence of a small net gas flow, the socalled DC gas flow, in the system. Numerical experiments show that the overshoot is sensitive to the velocity and temperature boundary conditions, the initial conditions and the grid refinements. In the one-dimensional model it never disappears completely. Surprisingly, in our two-dimensional results it is only present in the first cycles.

Using our model we can compute the position of the gas particles moving due to the pressure oscillations inside the pulse tube. Figure 5.9 shows the temperature of the gas versus particle position during one cycle for both driving pressures. From these two figures, it is seen that the distance travelled by the gas particles depends on the pressure wave form and the temperature variations during the cycle are different at different locations in the tube. Figure 5.10 shows pressure versus displacement for the two driving pressures at two different positions.

\subsubsection{Mass flow and enthalpy flow}

If velocity and temperature are known, we can compute the mass flow, the enthalpy flow and the time-averages of these.

The mass flow is

$$
\dot{\mathrm{m}}=A_{\mathrm{t}} \rho \mathrm{u} .
$$

Figure 5.11 shows the mass flow at the cold and hot end for the sinusoidal and trapezoidal pressure variations after 9 cycles. The mass flow at the cold end is about ten times larger, because the cold gas travels faster and has larger density. Note that the net mass flow must be zero. We want to emphasize that the mass flow is affected by the velocity boundary condition (3.6), (2.52) prescribed at the hot end. Even a small variation in this boundary condition causes a net mass flow, in which case the cycle steady-state cannot be reached.

The time-averaged enthalpy flow is computed from

$$
\overline{\mathrm{H}}=\frac{1}{t_{c}} \int_{0}^{t_{c}} c_{p} \dot{m} T d t,
$$

where $t_{c}$ is the cycle period. The analysis in [66, p. 63] gives an analytical estimate for the cycle-averaged enthalpy flow, valid for small amplitude pressure oscillations,

$$
\overline{\mathrm{H}}=a C_{o r} \bar{p}^{2} \text { with } a=\frac{1}{t_{c}} \int_{0}^{t_{c}} P^{2}(t) d t .
$$

The parameter a depends on the function $\mathrm{P}(\mathrm{t})$ : for the sinusoidal pressure variation 


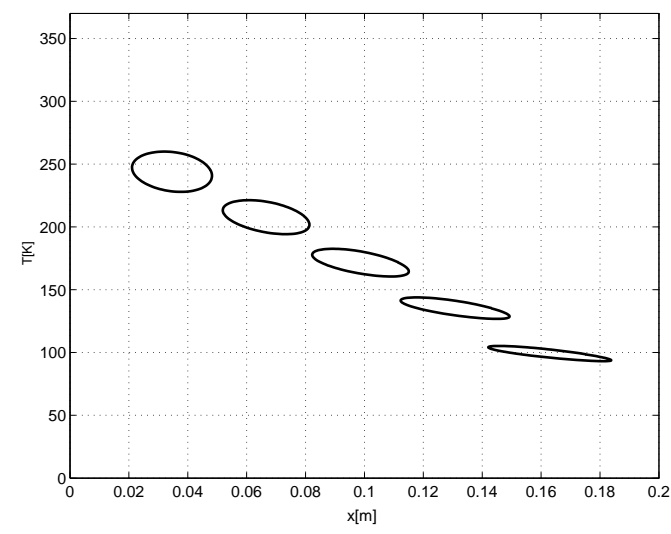

(a) sinusoidal driving pressure

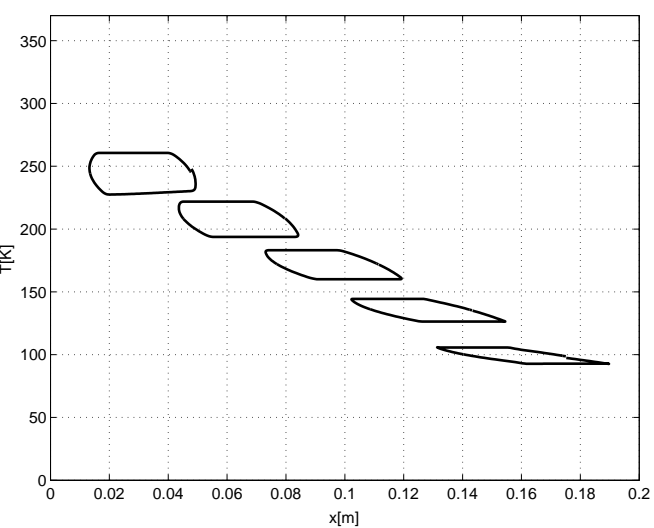

(b) trapezoidal driving pressure

Figure 5.9: Temperature of gas particles versus position for two different driving pressures.

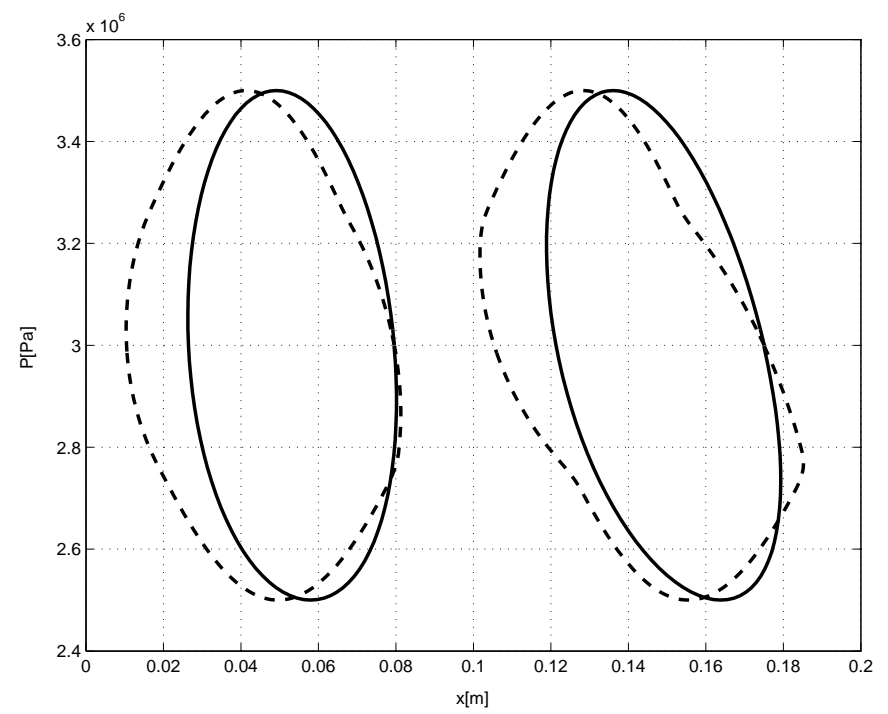

Figure 5.10: Pressure versus position of gas particles (solid line: sinusoidal pressure, dashed line: trapezoidal pressure). 


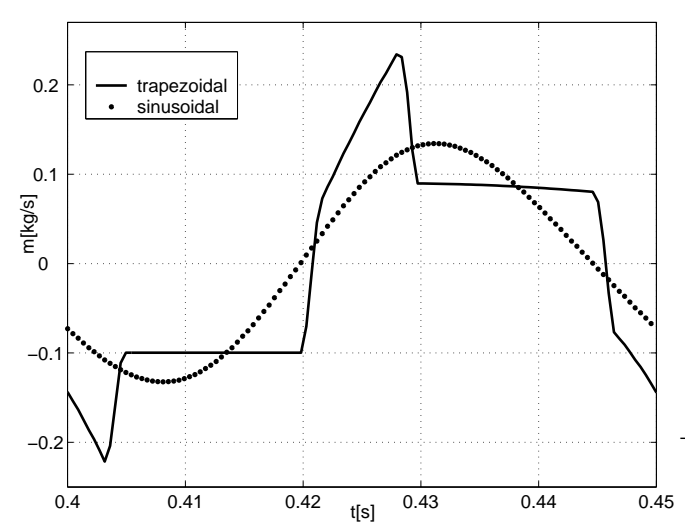

(a) cold end

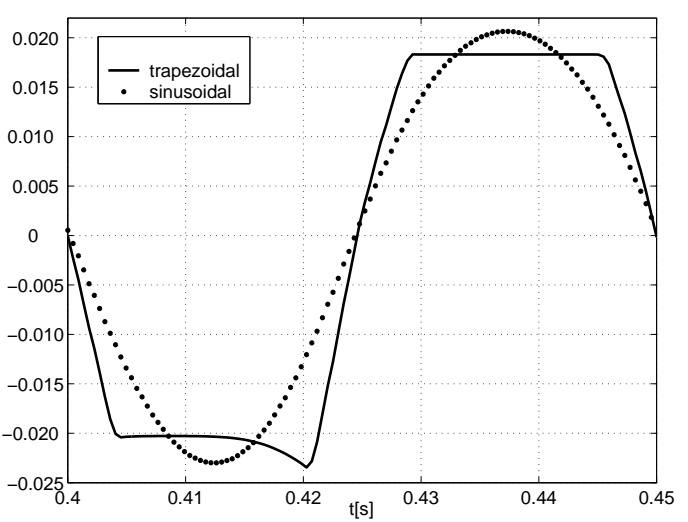

(b) hot end

Figure 5.11: Mass flow at the cold and hot ends for two different driving pressures, net mass flow $\approx 10^{-5}[\mathrm{~kg} / \mathrm{s}]$.

$a=1 / 2$, for the trapezoidal pressure variation $a=7 / 9$, which directly follows from

$$
P(t)= \begin{cases}6 t / \pi & \text { if } t<\pi / 6 \\ 1 & \text { if } \pi / 6 \leq t \leq 5 \pi / 6 \\ -6 t / \pi+6 & \text { if } 5 \pi / 6<t \leq \pi\end{cases}
$$

and the integration of $P^{2}(t)$ over the cycle. These results show that the shape of the pressure oscillations influences the magnitude of the enthalpy flow in the tube and therefore affects the refrigeration power. An important quantity with respect to refrigeration is the coefficient of performance. It is defined as the refrigeration power divided by the work input and it is independent of the shape of the pressure oscillations [66, p. 39]. The trapezoidal pressure has more refrigeration power, but producing this pressure variation costs more input power.

In the Tables 5.1 and 5.2 numerical values and analytical estimates for the timeaveraged enthalpy flow for the two different driving pressures of the same amplitude are presented. The direction of the enthalpy flow is shown by its sign: a minus sign means flow from the cold end to the hot end. The numerical results are averaged over the first and second halves of the cycle. The net enthalpy flows at the cold end and at the hot end are nearly the same in the one-dimensional simulation. The heat transfer between the gas and the wall is not included in the model and, ideally, there should be a very small enthalpy flow loss due to gas conduction. The order of magnitude can be estimated by

$$
\overline{\mathrm{H}}_{\mathrm{C}}-\overline{\mathrm{H}}_{\mathrm{H}} \approx \mathrm{k}_{\mathrm{g}} \mathrm{A}_{\mathrm{t}} \frac{\mathrm{T}_{\mathrm{H}}-\mathrm{T}_{\mathrm{C}}}{\mathrm{L}}=0.4[\mathrm{~W}] .
$$




\begin{tabular}{cccc}
\hline & $\begin{array}{c}\text { first half of the cycle } \\
\mathrm{P}(\mathrm{t})>0\end{array}$ & $\begin{array}{c}\text { second half of the cycle } \\
\mathrm{P}(\mathrm{t})<0\end{array}$ & cycle-averaged \\
\hline \hline hot end & $-23656.1 \mathrm{~W}$ & $22388.4 \mathrm{~W}$ & $-1267.7 \mathrm{~W}$ \\
middle & $-23656.1 \mathrm{~W}$ & $22388.5 \mathrm{~W}$ & $-1267.6 \mathrm{~W}$ \\
cold end & $-23655.9 \mathrm{~W}$ & $22388.6 \mathrm{~W}$ & $-1267.3 \mathrm{~W}$ \\
\hline & & & $-1250 \mathrm{~W}$ \\
\hline
\end{tabular}

Table 5.1: Time-averaged enthalpy flow $\overline{\mathrm{H}}$ for sinusoidal pressure variation.

\begin{tabular}{cccc}
\hline & $\begin{array}{c}\text { first half of the cycle } \\
\mathrm{P}(\mathrm{t})>0\end{array}$ & $\begin{array}{c}\text { second half of the cycle } \\
\mathrm{P}(\mathrm{t})<0\end{array}$ & cycle-averaged \\
\hline \hline hot end & $-30818.4 \mathrm{~W}$ & $28846.7 \mathrm{~W}$ & $-1971.7 \mathrm{~W}$ \\
middle & $-30610.6 \mathrm{~W}$ & $28642.0 \mathrm{~W}$ & $-1968.6 \mathrm{~W}$ \\
cold end & $-30403.1 \mathrm{~W}$ & $28437.9 \mathrm{~W}$ & $-1965.2 \mathrm{~W}$ \\
\hline & & & $-1944 \mathrm{~W}$ \\
\hline
\end{tabular}

Table 5.2: Time-averaged enthalpy flow $\overline{\mathrm{H}}$ for trapezoidal pressure variation. 
The numerical results with the sinusoidal pressure are more accurate because the analytical expressions for pressure $(\sin (t))$ and pressure derivative $(\cos (t))$ have been used. For the trapezoidal pressure, we have used sampled data. This gives an additional error associated with numerical interpolation and differentiation. Such an error would also occur when using measured (i.e. sampled) pressures.

\subsection{Two-dimensional results}

The two-dimensional model allows a detailed study of heat transfer between gas and wall and gives a quantitative prediction of energy transport in the system.

The same physical parameters as in the one-dimensional computation have been used in the two-dimensional simulation. The numerical parameters for the simulation described in this section are given in Table 5.3. With these values a one-cycle computation takes about 10 minutes on a standard PC with Intel's Xeon $2.66 \mathrm{GHz}$ processor and with $1 \mathrm{~GB}$ of internal memory.

\begin{tabular}{ccc}
\hline Symbol & Definition & Value \\
& & \\
\hline & & \\
$N_{z}$ & number of points in $z$ direction & 101 \\
$\mathrm{~N}_{r}$ & number of points in r direction & 45 \\
$\mathrm{~N}_{w}$ & number of points in the wall & 5 \\
$\mathrm{~N}_{\mathrm{t}}$ & number of time steps per cycle & 200 \\
$\mathrm{~N}_{\text {cycles }}$ & number of cycles & 100 \\
\hline
\end{tabular}

Table 5.3: Numerical parameters.

\subsubsection{Temperature and flow computations}

The temperature equations (4.1) for the gas and (4.4) for the wall, together with the boundary conditions (4.5) - (4.9), have been solved. The velocity equations have been decoupled from the temperature equation by using values from the previous time level. The velocity field has been computed from the equations (4.31) - (4.33) with boundary conditions (4.35) - (4.38). The prescribed velocity at the hot end is a plug flow varying with time. It is defined by the equations (2.48), (2.52). This is a realistic boundary condition from a physical point of view because of the usage of flow straighteners at the ends of the tube [69, p. 38]. For the numerical solution of the two-dimensional equations we use a uniform mesh in the $z$-direction and a nonuniform mesh, defined by equation (4.12) with parameter values $\mathcal{P}=1.8$ and $\Theta=2.0$, in the r-direction. A variable time step is chosen according to the CFL condition (4.18).

Temperature distributions and corresponding velocity fields at different parts of the third pressure cycle, $t=4 \pi, 4.5 \pi, 5 \pi$ and $5.5 \pi$, are presented in Figure 5.12. The 

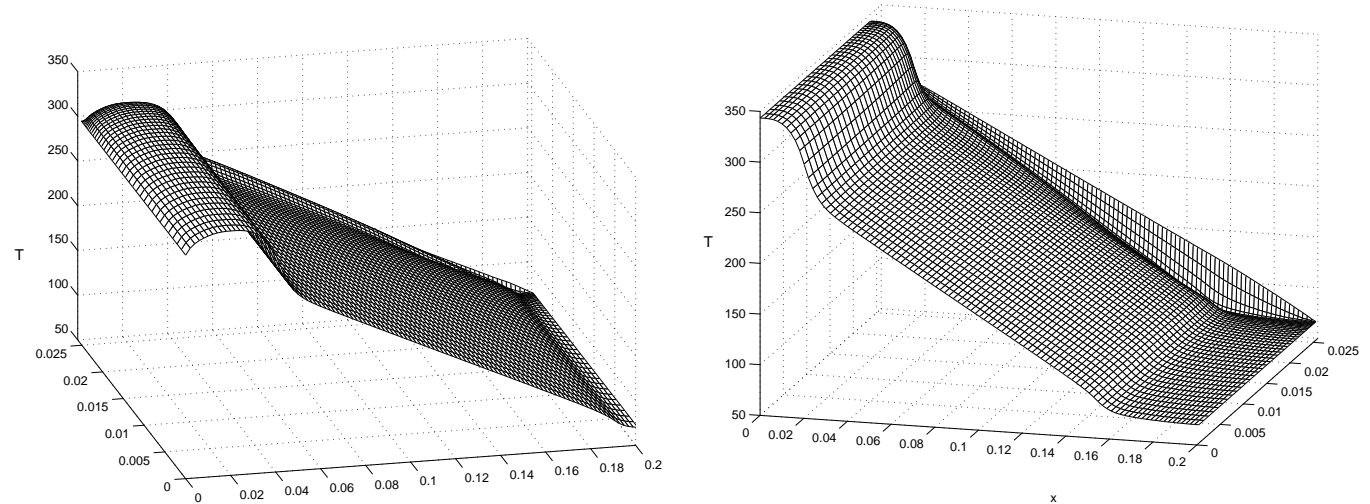

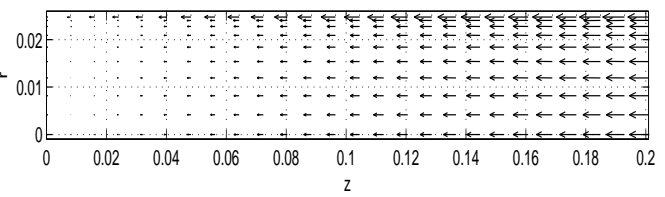

(a) $\mathrm{t}=4 \pi$
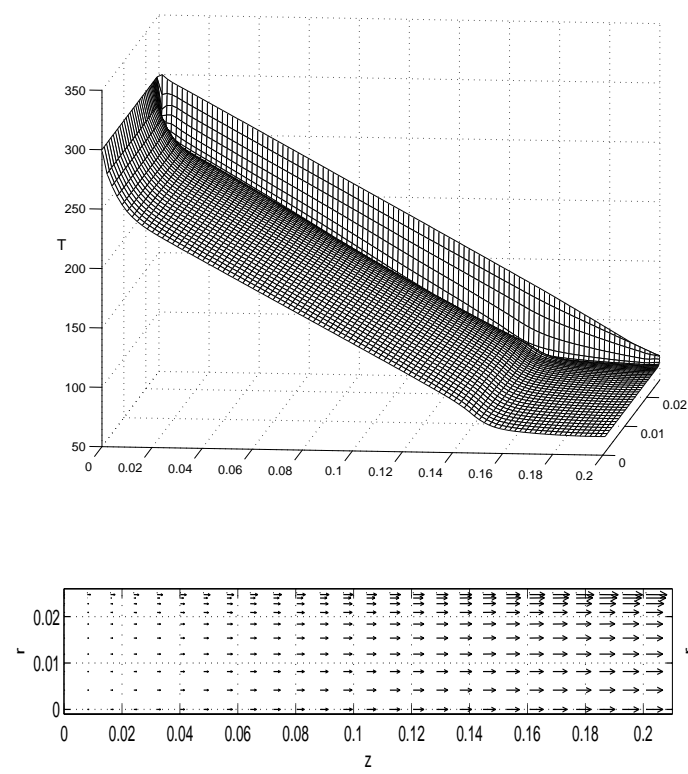

(c) $t=5 \pi$

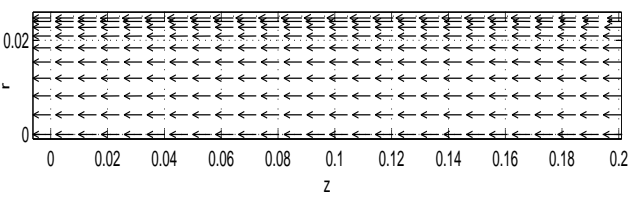

(b) $\mathrm{t}=4.5 \pi$
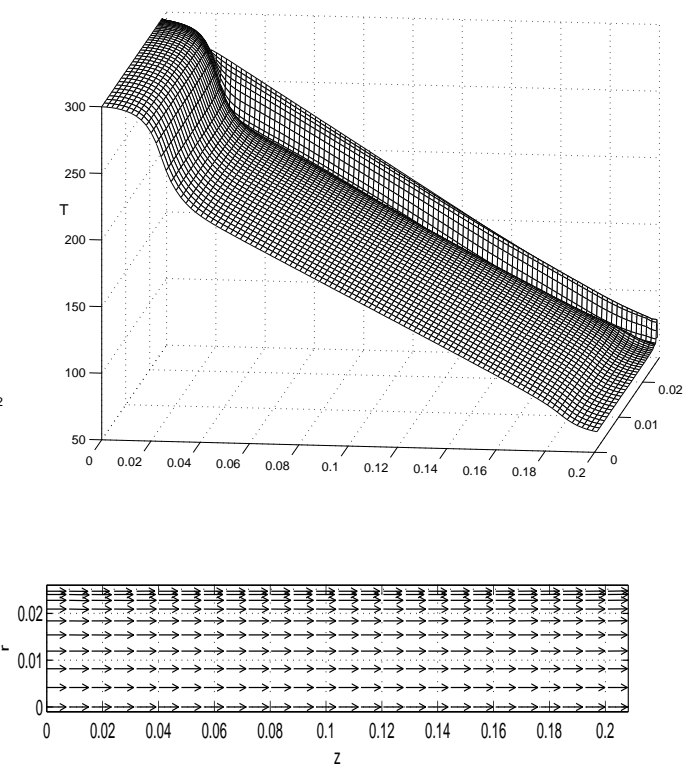

(d) $t=5.5 \pi$

Figure 5.12: Temperatures and velocity fields at four different times in the third pressure cycle. 


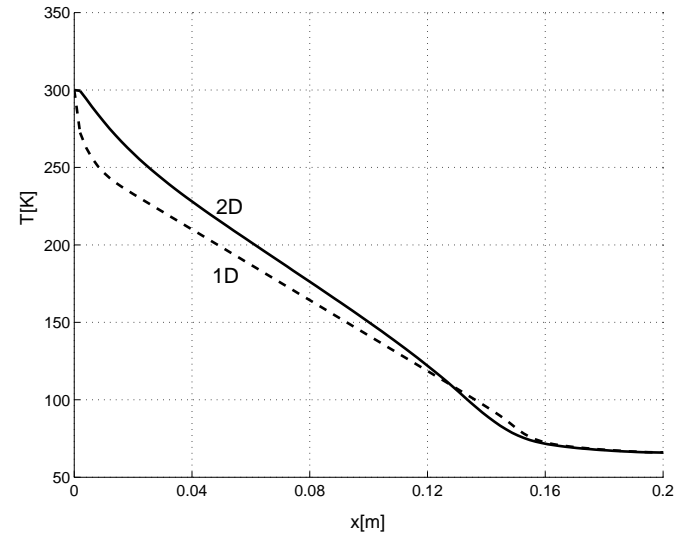

(a) $t=199 \pi$

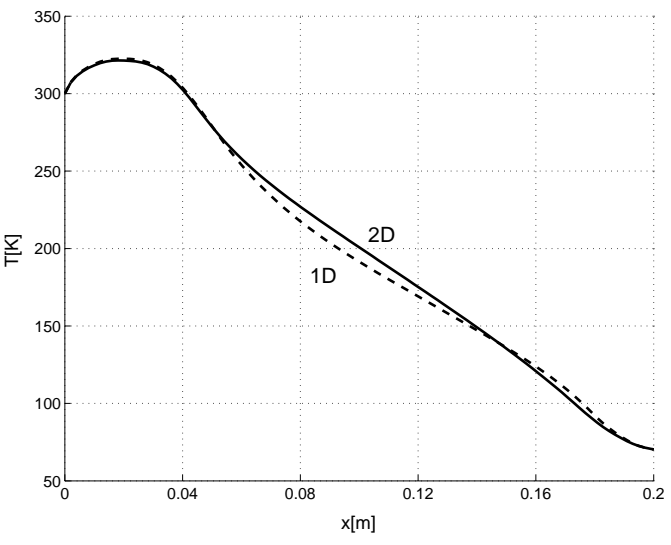

(b) $t=200 \pi$

Figure 5.13: Temperature at times $t=199 \pi$ and $t=200 \pi$, obtained with 1D and 2D models.

velocity fields in these graphs give the impression of one-directional flow. Indeed, the radial components of the velocities are close to zero. Small deviations from zero are observed only in the entrance regions near the wall. The temperatures in the core of the tube resemble the one-dimensional results in Figure 5.4. The temperature calculated by the one-dimensional model and the cross-sectional averaged temperature calculated from the two-dimensional model

$$
\mathrm{T}_{\mathrm{a} v}(z)=\frac{2}{\mathrm{R}_{0}^{2}} \int_{0}^{\mathrm{R}_{0}} \mathrm{~T}(z, \mathrm{r}) \mathrm{r} \mathrm{dr},
$$

are nearly the same at time $t=4 \pi$ : the influence of the wall is not visible in the beginning of the simulation. This comparison also provides a measure of confidence in the two-dimensional computations. The same comparison was done after 100 cycles, at times $t=199 \pi$ and $t=200 \pi$, as shown in Figure 5.13. After 100 cycles the influence of the wall is clearly visible. These graphs show that only the middle part of the gas, called the gas piston, is significantly affected by the wall.

The temperature at the cold end and at the hot end, averaged over the cross-section, for the first cycles are shown in Figure 5.14. The overshoot, discussed in Section 5.1.2, is present only in the first cycles, it disappears after 50 cycles.

Variations of velocity and temperature during the third cycle at midpoint $z=\mathrm{L} / 2$ are given in Figure 5.15, where the region near the wall is zoomed in (bottom graphs). The uniform profiles outside the thermal and viscous boundary layers show that the gas in the core is essentially adiabatic. Near the wall the well known Richardson's annular effect is clearly seen: both in the velocity profiles and in the temperature profiles. 


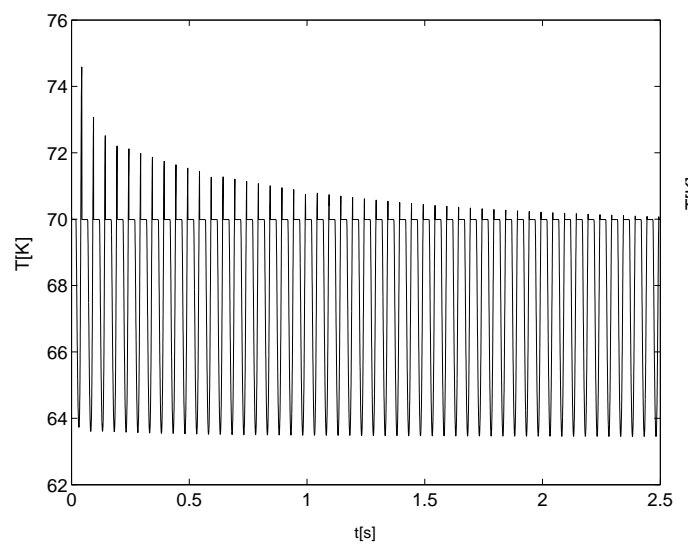

(a) cold end

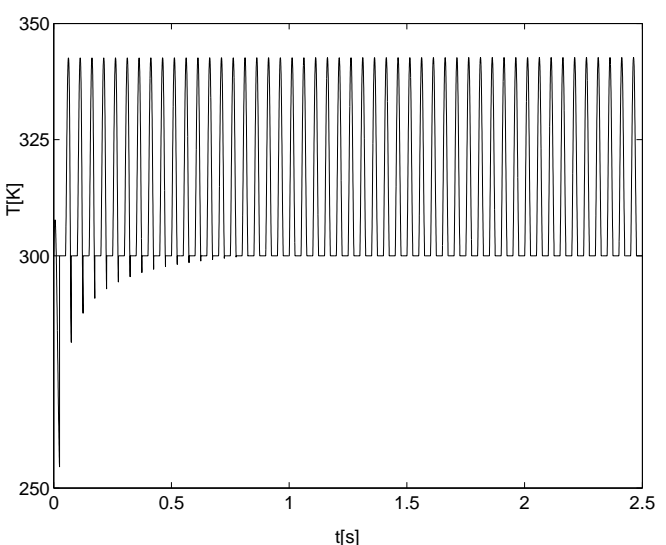

(b) hot end

Figure 5.14: Temperature, averaged over the cross-section, at cold and hot ends for the first 50 cycles.
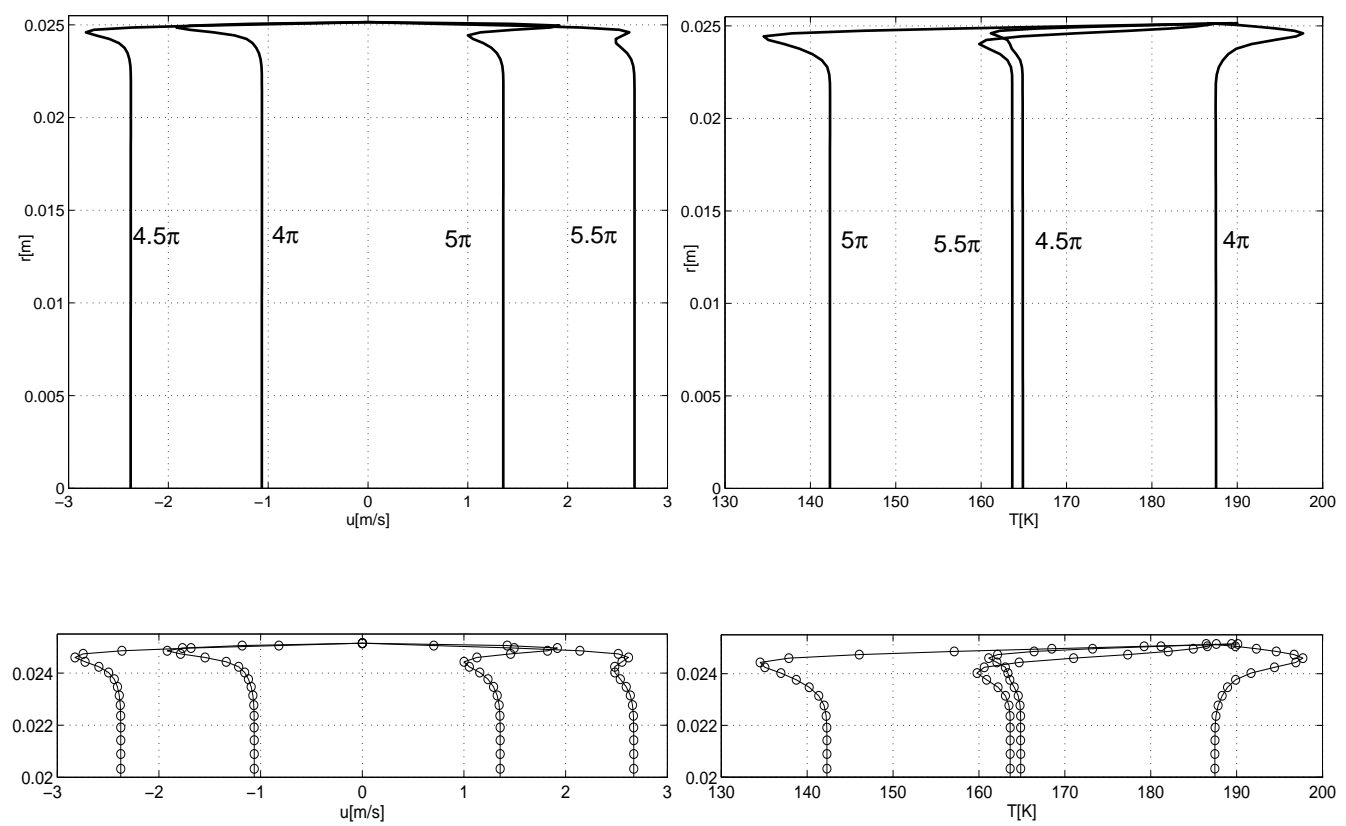

(a) velocity

(b) temperature

Figure 5.15: Radial temperature and velocity profiles at four different times in the third pressure cycle. 


\begin{tabular}{ccc}
\hline Symbol & Definition & Value \\
& & \\
\hline$N_{z}$ & number of points in $z$ direction & 101 \\
$\mathrm{~N}_{r}$ & number of points in r direction & 25 \\
$\mathrm{~N}_{w}$ & number of points in the wall & 5 \\
$\mathrm{~N}_{\mathrm{t}}$ & number of time steps per cycle & 100 \\
$\mathrm{~N}_{\text {cycles }}$ & number of cycles & 2000 \\
\hline
\end{tabular}

Table 5.4: Numerical parameters.

\subsubsection{Fluid-wall interaction}

The ability of the two-dimensional model to predict the temperature of the gas and the wall at any moment in time allows us to evaluate the heat fluxes and to estimate the energy balance in the system. In this section we present results of much longer simulation times, the numerical parameters are given in Table 5.4. To speed up the simulations we have used a coarser grid in the r-direction and fewer time steps per cycle.

The fluid-wall interface heat flux is computed using the definition

$$
q_{i n t}(z)=-k_{g}\left(\frac{\partial T(z, r)}{\partial r}\right)_{r=R_{0}} .
$$

Positive interface heat flux means heat flow in the positive $r$-direction: from the gas to the wall . The heat fluxes due to axial heat conduction in the gas and in the wall are computed using cross-sectional averaged temperatures

$$
\begin{aligned}
& \mathrm{q}_{\mathrm{gas}}(z)=-\mathrm{k}_{\mathrm{g}} \frac{\partial \mathrm{T}_{\mathrm{av}}(z)}{\partial z}, \\
& \mathrm{q}_{w \mathrm{all}}(z)=-\mathrm{k}_{w} \frac{\partial \mathrm{T}_{\mathrm{av}}(z)}{\partial z} .
\end{aligned}
$$

The interface heat fluxes at four different times in the 2000th sinusoidal pressure cycle: $\pi / 2$ - maximum pressure (one quarter of the cycle), $\pi$ - pressure decreases (half of the cycle), $3 \pi / 2$ - minimum pressure (three quarters of the cycle), $2 \pi$ - pressure increases (full cycle), are presented in Figure 5.16(a). The interface heat flux at $t=\pi$ is negative, the gas has expanded and the wall is warmer than the gas. At $t=2 \pi$ the interface heat flux is positive, the gas has been compressed and the wall is colder than the gas. The heat fluxes due to axial conduction during the last computed cycle are given in Figure 5.16(b). These heat fluxes are most of the time positive, heat is transported from the hot end to the cold end. Comparing these two figures, we see 


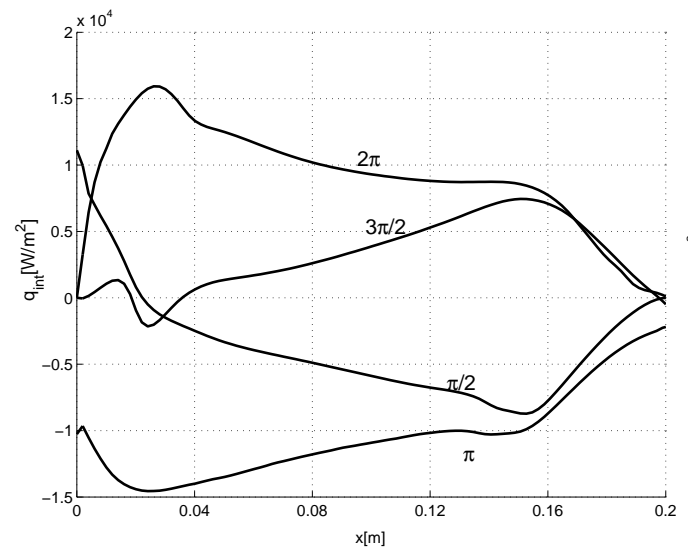

(a) interface heat flux

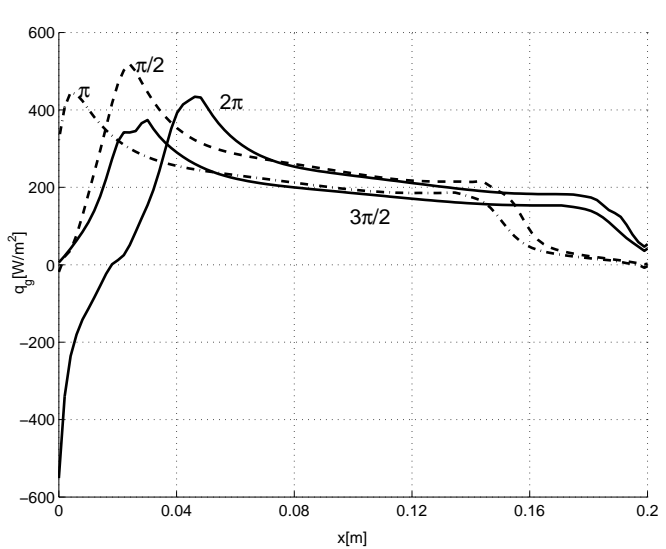

(b) axial gas heat flux

Figure 5.16: The interface fluxes and gas heat fluxes for different times during the 2000th cycle.

that the interface heat flux is of the order of $10^{4}\left[\mathrm{~W} / \mathrm{m}^{2}\right]$ and the axial gas heat flux is of the order of $10^{2}\left[\mathrm{~W} / \mathrm{m}^{2}\right]$.

The cycle-averaged heat fluxes at the interface, in the gas and in the wall are

$$
\begin{aligned}
\bar{q}_{\text {int }}(z) & =\frac{1}{t_{c}} \int_{0}^{t_{c}} q_{\text {int }} d t, \\
\bar{q}_{\text {gas }}(z) & =\frac{1}{t_{c}} \int_{0}^{t_{c}} q_{\text {gas }} d t, \\
\bar{q}_{\text {wall }}(z) & =\frac{1}{t_{c}} \int_{0}^{t_{c}} q_{\text {wall }} d t .
\end{aligned}
$$

The cycle-averaged interface heat flux as function of the axial position for different numbers of cycles is plotted in Figure 5.17(a). It shows how much heat is transferred from the gas to the wall (positive values) or from the wall to the gas (negative values) during one cycle. The heat transport between the wall and the gas is larger at the beginning of the simulation. As time proceeds the net amount of heat transported during one cycle becomes smaller and nearly constant in the gas piston region when the cyclic steady-state is approached. The cycle-averaged gas and wall heat fluxes, plotted in Figure 5.17(b) and Figure 5.17(c), change little with time. The enthalpy flow per unit area for different numbers of cycles as function of axial position, crosssectional averaged and time averaged according to

$$
\overline{\dot{h}}(z)=\overline{\dot{H}}(z) / A_{t}=\frac{c_{p}}{t_{c}} \int_{0}^{t_{c}}\left[\frac{2}{R_{0}^{2}} \int_{0}^{R_{0}} \rho(z, r) u(z, r) T(z, r) r d r\right] d t,
$$




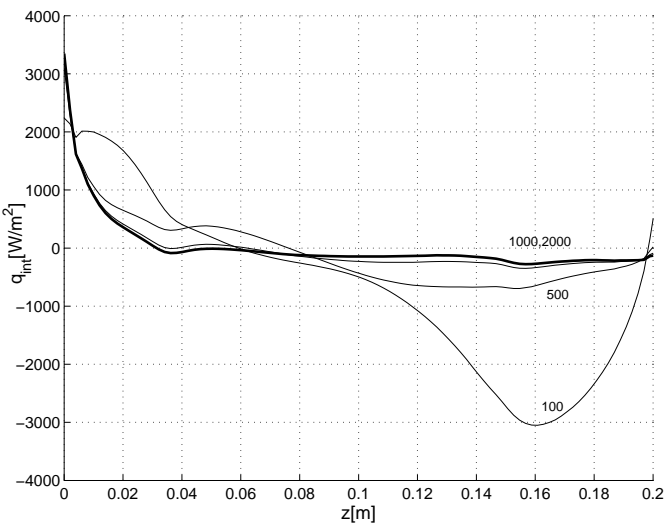

(a) interface heat flux

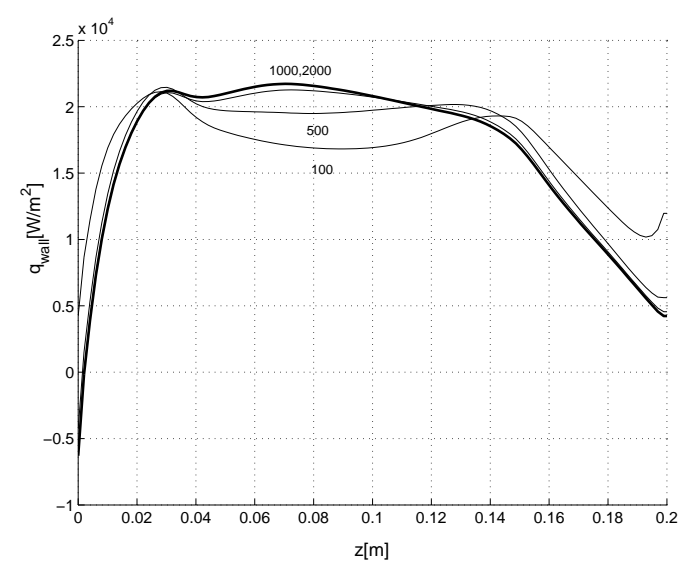

(c) wall axial heat flux

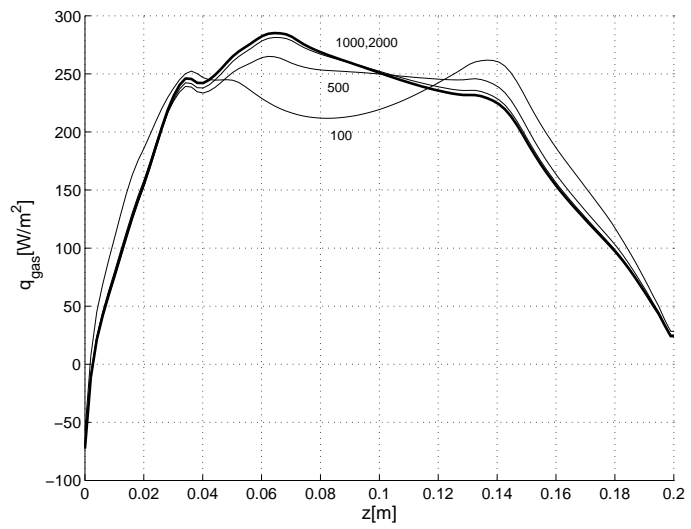

(b) gas axial heat flux

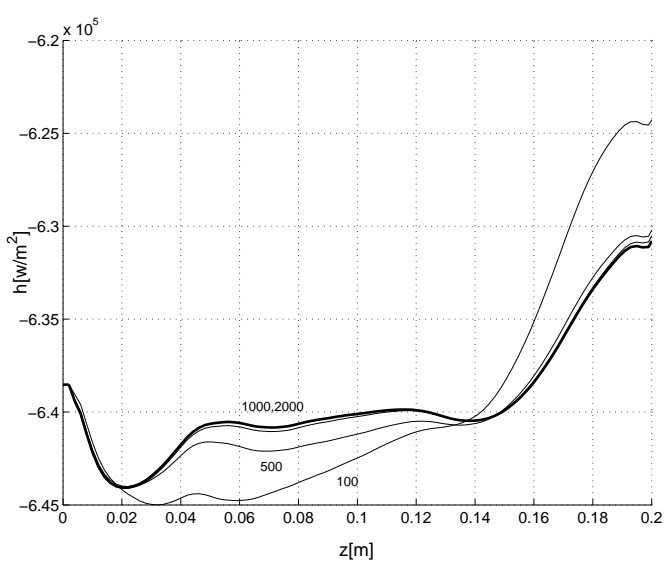

(d) enthalpy flow

Figure 5.17: The cycle-averaged interface, gas and wall heat fluxes and the enthalpy flow for different numbers of cycles. 


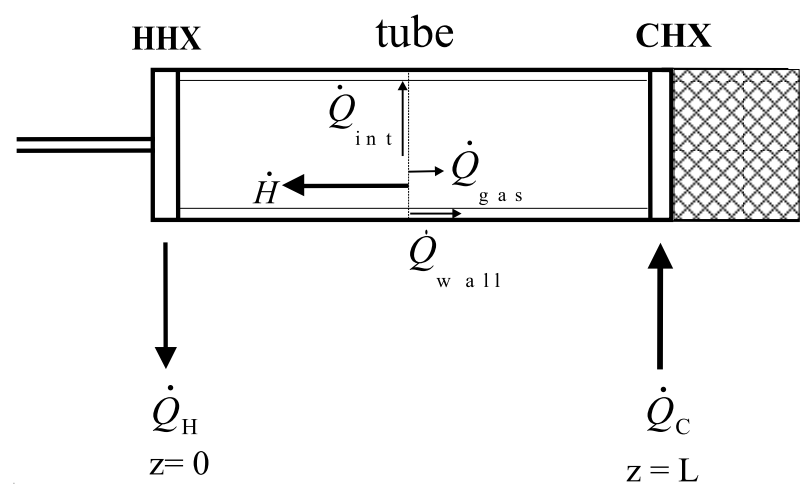

Figure 5.18: Cycle-averaged energy transport in the tube.

is given in Figure 5.17(d). From all these graphs we see that the difference between the 1000th and 2000th cycle is very small and the system has reached the cyclic steadystate.

The energy transport in the tube is schematically plotted in Figure 5.18. In steadystate the total energy flow along the tube is the sum of the enthalpy flow and the heat flows due to conduction in the gas and in the wall:

$$
\overline{\mathrm{E}}=\overline{\mathrm{H}}+\overline{\mathrm{Q}}_{g a s}+\overline{\mathrm{Q}}_{w a l l} \text {. }
$$

The heat flows due to conduction in the gas, $\overline{\mathrm{Q}}_{\mathrm{gas}}=A_{t} \overline{\mathrm{q}}_{\mathrm{gas}}$, and conduction in the wall, $\overline{\mathrm{Q}}_{w a l l}=A_{w} \overline{\mathrm{q}}_{w a l l}$, are plotted in Figure 5.19(a,b). They are comparable to the expected order of magnitude that can be estimated by

$$
\begin{aligned}
& \overline{\mathrm{Q}}_{\mathrm{gas}} \approx \mathrm{k}_{\mathrm{g}} \mathrm{A}_{\mathrm{g}} \frac{\mathrm{T}_{\mathrm{H}}-\mathrm{T}_{\mathrm{C}}}{\mathrm{L}} \approx 0.4[\mathrm{~W}], \\
& \overline{\mathrm{Q}}_{w \mathrm{all}} \approx \mathrm{k}_{w} A_{w} \frac{\mathrm{T}_{\mathrm{H}}-\mathrm{T}_{\mathrm{C}}}{\mathrm{L}} \approx 2.7[\mathrm{~W}] .
\end{aligned}
$$

$\overline{\mathrm{Q}}_{\mathrm{gas}}$ and $\overline{\mathrm{Q}}_{\text {wall }}$ are much smaller than the enthalpy flow $\overline{\mathrm{H}}$, which has an average value of $-1279[\mathrm{~W}]$ per cycle. The enthalpy flow along the tube lifts heat from the cold end towards the hot end. The enthalpy flow $\overline{\dot{H}}$ and the total energy flow along the tube $\overline{\dot{E}}$ as functions of axial position are given in Figure 5.19(c). The energy flow is about constant in the middle of the tube and deviates up to $1.4 \%$ from the average value in the boundary regions because of numerical error. The interface flux per unit length $\left(\pi D \bar{q}_{i n t}\right)$ as function of axial position is given in Figure $5.19(\mathrm{~d})$. Heat is withdrawn from the gas when its temperature is higher than the wall temperature (positive flux) and heat is retrieved to the gas when it is colder (negative flux). The 


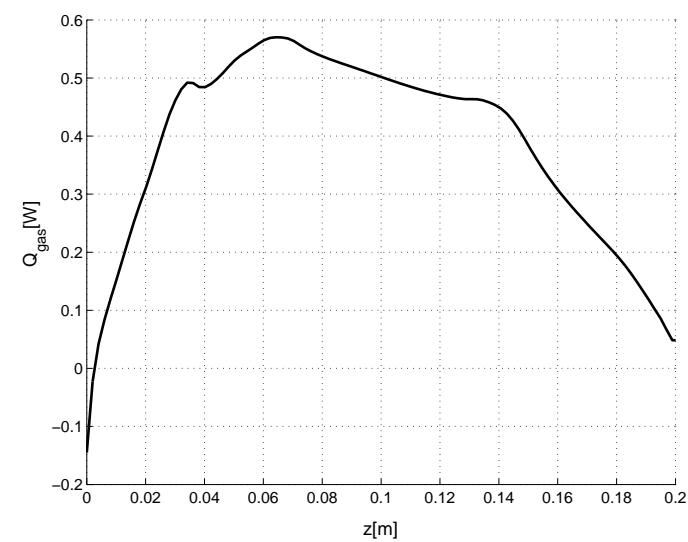

(a) gas axial heat flow

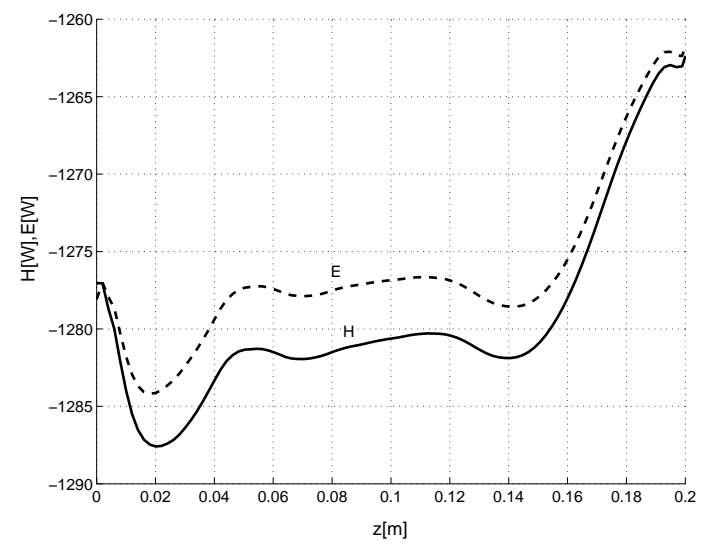

(c) axial enthalpy and energy flows

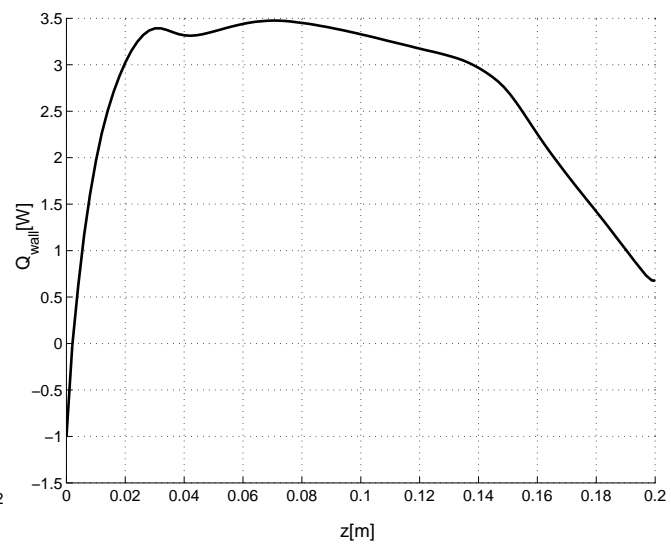

(b) wall axial heat flow

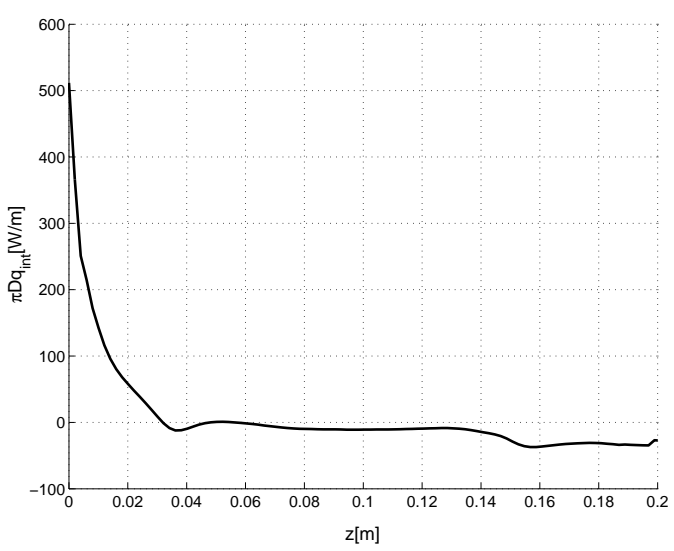

(d) interface heat flux per unit length

Figure 5.19: Gas axial heat flow (a), wall axial heat flow (b), axial enthalpy (solid line) and energy (dashed line) flows (c) and interface heat flux per unit length (d). 


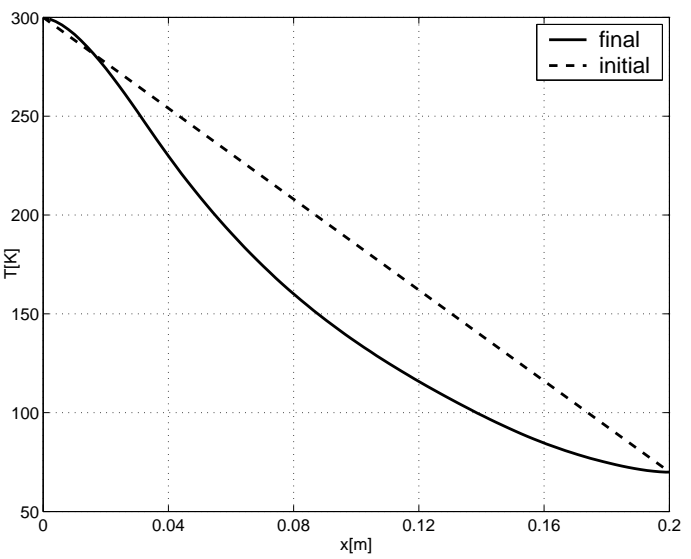

Figure 5.20: Wall temperature at the beginning of the simulation (dashed line) and after 2000 cycles (solid line). The solid line also represents the average gas temperature after 2000 cycles.

net gas-to-wall heat transfer amounts

$$
\overline{\mathrm{Q}}_{\text {int }}=\int_{0}^{\mathrm{L}} \pi \mathrm{D} \overline{\mathrm{q}}_{\text {int }}(z) \mathrm{d} z \approx 1.6[\mathrm{~W}]
$$

per cycle. This shows how much heat is transported from the gas to the wall, which is confirmed, see Figure 5.19(b), by the energy balance for the wall:

$$
\overline{\mathrm{Q}}_{\text {int }}=\overline{\mathrm{Q}}_{w \mathrm{all}}(z=\mathrm{L})-\overline{\mathrm{Q}}_{w a l l}(z=0) \approx 0.7[\mathrm{~W}]+1.0[\mathrm{~W}]=1.7[\mathrm{~W}] .
$$

According to the above considerations the heat flows in the tube are much smaller than the enthalpy flows. From a physical point of view this means that heat conduction is not a relevant loss mechanism in the investigated pulse-tube.

The wall temperature at the beginning of the simulation and after 2000 cycles is displayed in Figure 5.20. After cyclic steady-state is reached the wall temperature is equal to the cross-sectionally averaged and time-averaged gas temperature. The prediction of the wall temperature is important from a validation point of view. It can be easily measured in physical experiments. 


\section{Concluding remarks and future work}

The newly developed model simulates oscillating flow and heat transfer in the tube section of a pulse-tube refrigerator. The prediction of heat transfer in oscillatory flow regimes is of great importance in the area of thermal engineering. In this study we concentrated on one particular system: the orifice pulse-tube refrigerator. However, the developed mathematical model has been kept general and can be applied for studying unsteady flow and heat transfer in a wide range of applications. With respect to the pulse tube, high frequencies, arbitrary pressure variations, different length to diameter ratios can be simulated.

The main assumptions in our one-dimensional model are: laminar flow, ideal gas, Newtonian fluid, no external forces. The one-dimensional model can predict velocity, temperature, mass flow and enthalpy flow in the tube. Using a state-of-the-art flux-limiter scheme the steep temperature gradients that occur in the tube are preserved. The model allows the incorporation of measured data. It is more versatile than the widely used harmonic analysis and it is computationally not expensive. A serious drawback of the one-dimensional model is its inability to describe fluid-wall interactions, which play an important role in the cooling process.

Our two-dimensional model includes radial thermal and viscous effects. Additional assumptions in the two-dimensional computations are uniform viscosity and thermal conductivity. The two-dimensional model allows a detailed study of heat transfer between gas and wall and it gives a quantitative prediction of energy transport in the system.

The proposed model and numerical algorithms have been implemented in a simulation tool. The implementation has been done in $\mathrm{C}++$, using the computational platform NumLab [12]. The workbench NumLab runs on Linux and Silicon Graphics systems and provides an interface for efficient software development: reuse of existing libraries in an unaltered way, data exchange with MATLAB, Mathematica, LATEX and visualisation of results. Our simulation tool gives tremendous gain in com- 
putational time when compared to commercial CFD software. The results reported in [88] required 16 hours per cycle on a $\mathrm{PC}$ with $1 \mathrm{GHz}$ processor whereas we needed only several minutes for a one-cycle computation employing approximately the same number of grid points and time steps.

Though the newly developed model is able to predict oscillating flow and heat transfer in a pulse tube, there is considerable room for further investigations. Possible areas for future studies include:

- Detailed parametric study and optimisation. This would estimate the influence on the cooling power the major physical parameters of the system, such as operating frequency, pressure amplitude, length to diameter ratio, flow conductance of the orifice, cold end temperature. To be able to do this, the simulation tool should be optimised with respect to time and memory usage to perform the large amount of calculations within reasonable time. A user-friendly interface is an important part of the design tool and needs to be added.

- Extending the two-dimensional model to temperature-dependent thermophysical properties, such as viscosity $\mu(T)$, thermal conductivity $k_{g}(T)$ and heat capacity $c_{p}(T)$. The heat transfer characteristics of a cryogenic fluid change significantly with temperature. The constant-property analysis, valid for many ambient temperature applications, is often inaccurate when applied to cryogenic systems. The temperature-dependent viscosity $\mu(T)$ allows to study acoustic streaming effects.

- Extending the model for different geometries. Several theoretical and experimental studies have been done on tapered pulse tubes, see [3], [4], [52], [64], [91]. It was found that the performance of pulse tube refrigerators can be improved, i.e. decreasing the minimum temperature or increasing the cooling power at the same refrigerating temperature, when using a tapered tube with optimal cone angle.

- Incorporating other parts of the pulse-tube refrigerator, such as regenerator and heat exchangers, in the model. It will give a more complete design tool.

- Three-dimensional modelling can give an additional insight on gravity effect, asymmetry, turbulence and external forces effect such as vibration. 


\section{Appendix A. Parameters for a typical single-inlet pulse-tube refrigerator}

\begin{tabular}{|c|c|c|}
\hline Symbol & Definition & Typical Value \\
\hline $\operatorname{Re}$ & $\bar{\rho} \bar{u}^{2} / \bar{\mu} \omega$ & $4.2 \times 10^{3}$ \\
\hline Мa & $\overline{\mathrm{u}} /\left(\mathrm{p}_{\mathrm{a} v} / \bar{\rho}\right)^{1 / 2}$ & $1.9 \times 10^{-3}$ \\
\hline $\mathrm{M}$ & $\overline{\mathrm{u}} /(\overline{\mathrm{p}} / \bar{\rho})^{1 / 2}$ & $4.6 \times 10^{-3}$ \\
\hline $\operatorname{Pr}$ & $c_{p} \bar{\mu} / \bar{k}_{g}$ & 0.66 \\
\hline $\mathrm{Pe}$ & $\operatorname{RePr}$ & $2.6 \times 10^{3}$ \\
\hline $\mathrm{Ec}$ & $\bar{u}^{2} / c_{p} T_{a}$ & $1.5 \times 10^{-6}$ \\
\hline $1 / \mathrm{M}^{2}$ & $\bar{p} / \bar{\rho} \bar{u}^{2}$ & $4.7 \times 10^{4}$ \\
\hline $1 / \operatorname{Re}$ & $\bar{\mu} \omega / \bar{\rho} \bar{u}^{2}$ & $2.5 \times 10^{-4}$ \\
\hline $1 / \mathrm{Pe}$ & $\bar{k}_{\mathrm{g}} \omega / \bar{\rho} c_{p} \bar{u}^{2}$ & $3.6 \times 10^{-4}$ \\
\hline Fo & $\overline{\mathrm{k}}_{w} \omega / \rho_{w}^{-} \mathrm{c}_{w} \overline{\mathrm{u}}^{2}$ & $2.6 \times 10^{-4}$ \\
\hline $\mathcal{A}$ & $\mathrm{p}_{\mathrm{a} v} / \overline{\mathrm{p}}$ & 6 \\
\hline $\mathcal{B}$ & $\bar{p} / \bar{\rho} R_{m} T_{a}$ & 0.17 \\
\hline $\mathcal{C}$ & $C_{o r} \bar{p} / A_{t} \bar{u}$ & 1.67 \\
\hline $\mathcal{D}$ & $C_{o r} c_{p} \bar{p} / V_{B} c_{\nu} \omega$ & $1 / 24 \pi$ \\
\hline $\mathcal{E}$ & $\overline{\mathrm{p}}_{\mathrm{b}} / \overline{\mathrm{p}}$ & 6.048 \\
\hline$\gamma$ & $c_{p} / c_{v}$ & $5 / 3$ \\
\hline
\end{tabular}

Table 6.1: Dimensionless numbers for a typical single-inlet pulse-tube refrigerator. 


\begin{tabular}{|c|c|c|}
\hline Symbol & Definition & Value \\
\hline$f$ & frequency & $20 \mathrm{~Hz}$ \\
\hline$\omega$ & angular frequency & $125.66 \mathrm{~s}^{-1}$ \\
\hline $\bar{\rho}$ & gas density & $4.7 \mathrm{~kg} \mathrm{~m}^{-3}$ \\
\hline $\bar{\rho}_{w}$ & wall density & $7850 \mathrm{~kg} \mathrm{~m}^{-3}$ \\
\hline $\bar{u}$ & gas velocity & $1.5 \mathrm{~m} \mathrm{~s}^{-1}$ \\
\hline $\bar{\mu}$ & viscosity & $2.0 \times 10^{-5} \mathrm{~Pa} \mathrm{~s}$ \\
\hline$\overline{\mathrm{k}}_{g}$ & gas thermal conductivity & $1.58 \times 10^{-1} \mathrm{~W} \mathrm{~m}^{-1} \mathrm{~K}^{-1}$ \\
\hline $\bar{k}_{w}^{9}$ & wall thermal conductivity & $14.67 \mathrm{~W} \mathrm{~m}^{-1} \mathrm{~K}^{-1}$ \\
\hline$c_{p}$ & gas specific heat capacity & $5.2 \times 10^{3} \mathrm{~J} \mathrm{~kg}^{-1} \mathrm{~K}^{-1}$ \\
\hline$c_{w}$ & wall specific heat capacity & $4 \times 10^{2} \mathrm{~J} \mathrm{~kg}^{-1} \mathrm{~K}^{-1}$ \\
\hline $\bar{p}$ & pressure oscillation amplitude & $5 \times 10^{5} \mathrm{~Pa}$ \\
\hline$p_{a v}$ & average pressure & $3 \times 10^{6} \mathrm{~Pa}$ \\
\hline $\mathrm{T}_{\mathrm{a}}$ & ambient temperature & $300 \mathrm{~K}$ \\
\hline $\mathrm{R}_{\mathrm{m}}$ & specific gas constant & $2.1 \times 10^{3} \mathrm{~J} \mathrm{~kg}^{-1} \mathrm{~K}^{-1}$ \\
\hline$A_{t}$ & cross-sectional area of tube & $2.0 \times 10^{-3} \mathrm{~m}^{2}$ \\
\hline $\mathrm{D}$ & diameter of the tube & $5.0 \times 10^{-2} \mathrm{~m}$ \\
\hline $\mathrm{C}_{\mathrm{or}}$ & flow conductance of the orifice & $10^{-8} \mathrm{~m}^{3} \mathrm{~Pa}^{-1} \mathrm{~s}^{-1}$ \\
\hline $\mathrm{L}$ & length of tube & $0.2 \mathrm{~m}$ \\
\hline$l$ & wall thickness & $1 \mathrm{~mm}$ \\
\hline $\mathrm{T}_{\mathrm{H}}$ & hot end temperature & $300 \mathrm{~K}$ \\
\hline $\mathrm{T}_{\mathrm{C}}$ & cold end temperature & $70 \mathrm{~K}$ \\
\hline$V_{b}$ & buffer volume & $5 \times 10^{-3} \mathrm{~m}^{3}$ \\
\hline
\end{tabular}

Table 6.2: Physical data for a typical single-inlet pulse-tube refrigerator. 


\section{Bibliography}

[1] B. F. Armaly, F. Durst, J. C. F. Pereira, and B. Schonung. Experimental and theoretical investigation of backward-facing step flow. J. Fluid Mech., 127:473-496, 1983.

[2] D. C. Arney and J. E. Flaherty. An adaptive local mesh refinement method for time dependent partial differential equations. Appl. Numer. Math., 5:257-274, 1989.

[3] S. H. Baek, E. S. Jeong, and S. Jeong. Two-dimensional model for tapered pulse tubes. Part 1: Theoretical modeling and net enthalpy flow. Cryogenics, 40:379385,2000 .

[4] S. H. Baek, E. S. Jeong, and S. Jeong. Two-dimensional model for tapered pulse tubes. Part 2: Mass streaming and streaming-driven enthalpy flow loss. Cryogenics, 40:387-392, 2000.

[5] I. E. Barton. Comparison of SIMPLE- and PISO-type algorithms for transient flows. Int. J. Numer. Meth. Fluids, 26:459-483, 1998.

[6] M. J. Berger and P. Colella. Local adaptive mesh refinement for shock hydrodynamics. J. Comp. Phys., 82:64-84, 1989.

[7] M. Berzins and J. M. Ware. Positive cell-centered finite volume discretization methods for hyperbolic equations on irregular meshes. Applied Numerical Mathematics, 16:417-438, 1995.

[8] R. B. Bird, W. E. Stewart, and E. N. Lightfoot. Transport Phenomena. John Wiley \& Sons, New York, 1960.

[9] G. J. Brereton and R. R. Mankbadi. Review of recent advances in the study of unsteady turbulent internal flows. Appl. Mech. Rev., 48(4):189-212, 1995.

[10] A. J. Chorin. A numerical method for solving incompressible viscous flow problems. J. Comp. Phys., 2:12-26, 1967.

[11] J. P. van Doormaal and G. D. Raithby. Enhancements of the SIMPLE method for predicting incompressible fluid flows. Numerical Heat Transfer, 7:147-163, 1984. 
[12] W. Drenth. A platform for numerical computations with special application to preconditioning. PhD thesis, Eindhoven University of Technology, 2003.

[13] D. M. Eckmann and J. B. Grotberg. Experiments on transition to turbulence in oscillatory pipe flow. J. Fluid Mech., 222:329-350, 1991.

[14] J. H. Ellison, C. A. Hall, and T. A. Porsching. An unconditionally stable convergent finite difference method for Navier-Stokes problems on curved domains. SIAM J. Num. Anal., 24:1233-1248, 1987.

[15] R. E. Ewing. Adaptive grid refinement for transient flow problems. In J. E. Flaherty, P. J. Paslow, M. S. Shephard, and J. D. Vasilakis, editors, Adaptive Methods for Partial Differential Equations, pages 194-205. SIAM, Philadelphia, 1989.

[16] J. H. Ferziger and M. Peric. Computational Methods for Fluid Dynamics. Springer, Berlin, 1996.

[17] J. E. Flaherty, P. K. Moore, and C. Ozturan. Adaptive overlapping grid methods for parabolic systems. In J. E. Flaherty, P. J. Paslow, M. S. Shephard, and J. D. Vasilakis, editors, Adaptive Methods for Partial Differential Equations, pages 176193. SIAM, Philadelphia, 1989.

[18] C. A. J. Fletcher. Computational Techniques for Fluid Dynamics. Springer, Berlin, 1988.

[19] D. K. Gartling. A test problem for outflow boundary conditions - flow over a backward-facing step. Int. J. Numer. Meth. Fluids, 11:953-969, 1990.

[20] W. E. Gifford and R. C. Longsworth. Pulse-tube refrigeration. Trans. ASME, $86: 264-268,1964$

[21] S.F. Grace. Oscillatory motion of viscous liquid in a long straight tube. London, Edinburgh and Dublin Phil. Mag., 31(5):933-939, 1928.

[22] P. M. Gresho, D. K. Gartling, J. R. Torzynski, K. A. Cliffe, K. H. Winters, T. J. Garratt, A. Spence, and J. W. Goodrich. Is the steady viscous incompressible two-dimensional flow over a backward-facing step at $\mathrm{Re}=800$ stable? Int. J. Numer. Meth. Fluids, 17:501-541, 1993.

[23] P. M. Gresho and R. L. Sani. On pressure boundary conditions for the incompressible Navier-Stokes equations. Int. J. Numer. Meth. Fluids, 7:1111-1145, 1987.

[24] W. D. Gropp and D. E. Keyes. Domain decomposition with local mesh refinement. SIAM J. Sci. Stat. Comput., 13:967-993, 1992.

[25] M. Hino, M. Sawamoto, and S. Takasu. Experiments on the transition to turbulence in an oscillating pipe flow. J. Fluid Mech., 75:193-207, 1976.

[26] C. Hirsch. Numerical computation of internal and external flows, Vol.2 Computational methods for inviscid and viscous flows. John Wiley \& Sons, Chichester, 1990. 
[27] B. van 't Hof, J. H. M. Ten Thije Boonkkamp, and R. M. M. Mattheij. Pressure correction for laminar combustion simulation. Combustion Science and Technology, 149:201-223, 1999.

[28] H. Hooijkaas. Miniature Stirling-type pulse-tube refrigerators. PhD thesis, Eindhoven University of Technology, 2000.

[29] H. W. G. Hooijkaas and A. A. J. Benschop. Pulse tube development using harmonic simulations. In R. G. Ross, editor, Cryocoolers 10, pages 359-367. Jr. Plenum Press, New York, 1999.

[30] Y. Hozumi and M. Murakami. Numerical study of gas dynamics inside of a pulse tube refrigerator. Advances in Cryogenic Engineering, 45:167-174, 2000.

[31] M. Iguchi and M. O. Carpinlioglu. Present state of the art on pulsatile flow theory ( part 1: laminar and transitional flow regimes). JSME Int. J., 42(3):384-397, 1999.

[32] F. P. Incropera and D. P. De Witt. Fundamentals of Heat and Mass Transfer. John Wiley \& Sons, Singapore, 1990.

[33] R. I. Issa. Solution of the implicitly discretised fluid flow equations by operatorspliting. J. Comput. Phys., 62:40-65, 1985.

[34] M. Jakob. Heat Transfer. John Wiley \& Sons, 1949.

[35] Y. L. Ju, C. Wang, and Y. Zhou. Numerical simulation and experimental verification of the oscillating flow in pulse tube refrigerator. Cryogenics, 38:169-176, 1998.

[36] Y. L. Ju, C. Wang, and Y. Zhou. Computational study of a $4 \mathrm{~K}$ two-stage pulse tube cooler with mixed Eulerian-Lagrangian method. Cryogenics, 41:49-51, 2001.

[37] R. Klein, N. Botta, T. Schneider, C. D. Munz, S. Roller, A. Meister, L. Hoffmann, T. Sonar, and W. E. Stewart. Asymptotic adaptive methods for multi-scale problems in fluid mechanics. Journal of Engineering Mathematics, 39:261-343, 2001.

[38] U. H. Kurzweg. Enhanced heat conduction in fluids subjected to sinusoidal oscillations. J. Heat Transfer, 107(5):459-462, 1985.

[39] U. H. Kurzweg. Enhanced heat conduction in oscillating viscous flows within parallel-plate channels. J. Fluid Mech., 156:291-300, 1985.

[40] U. H. Kurzweg, E. R. Lindgren, and B. Lothrop. Onset of turbulence in oscillating flow at low Womersley number. Physics of Fluids, 12:1972-1975, 1989.

[41] D. Y. Lee, S. J. Park, and S. T. Ro. Heat transfer in the thermally developing region of a laminar oscillating pipe flow. Cryogenics, 38(6):585-594, 1998.

[42] J. M. Lee, P. Kittel, K. D. Timmerhaus, and R. Radebaugh. Useful scaling parameters for the pulse tube. Advances in Cryogenic Engineering, 41:1347-1355, 1996. 
[43] J. M. Lee, P. Kittel, K. D. Timmerhaus, and R. Radebaugh. Higher order pulse tube modeling. In R. G. Ross, editor, Cryocoolers 9, pages 345-353. Jr. Plenum Press, New York, 1999.

[44] J. M. Lee, P. Kittel, K. D. Timmerhaus, and R. Radebaugh. Simple twodimensional corrections for one-dimensional pulse tube model. In R. G. Ross, editor, Cryocoolers 10, pages 351-358. Jr. Plenum Press, New York, 1999.

[45] P. Li and K. T. Yang. Mechanisms for the heat transfer enhancement in zeromean oscillatory flows in short channels. Int. J. Heat Mass Transfer, 43:3551-3566, 2000.

[46] I. A. Lyulina, R. M. M. Mattheij, A. S. Tijsseling, and A. T. A. M. de Waele. Numerical simulation of pulse-tube refrigerators: 1D model. In H. C. de Lange and A. A. van Steenhoven, editors, Heat transfer in unsteady and transitional flows: Proc. of the Eurotherm Seminar, vol.74, ISBN 90-386-2954-0, pages 91-97. Technische Universiteit Eindhoven, 2003.

[47] I. A. Lyulina, R. M. M. Mattheij, A. S. Tijsseling, and A. T. A. M. de Waele. Numerical simulation of pulse-tube refrigerators. International Journal of Nonlinear Sciences and Numerical Simulation, 5(1):79-88, Corigenda, 5(3):287, 2004.

[48] Y. Matsubara and J. L. Gao. Novel configuration of three-stage pulse tube refrigerator for temperatures below 4K. Cryogenics, 34:259-262, 1994.

[49] R. M. M. Mattheij, S. W. Rienstra, and J. H. M. ten Thije Boonkkamp. Partial Differential Equations, Modelling, Analysis, Computing. SIAM Press, Philadelphia, to appear.

[50] P. Merkli and H. Thomann. Transition to turbulence in oscillating pipe flow. J. Fluid Mech., 68:567-575, 1975.

[51] E. I. Mikulin, A. A. Tarasov, and M. P. Shkrebyonock. Low temperature expansion pulse tubes. Advances in Cryogenic Engineering, 29:629-637, 1984.

[52] H. C. Park, E. S. Jeong, and S. Jeong. Two-dimensional model for tapered pulse tubes. Part 3: Unsteady components of second-order mass flux and temperature. Cryogenics, 42:485-493, 2002.

[53] S. V. Patankar. Numerical Heat Transfer and Fluid Flow. Hemispere Publishing Corporation, New York, 1980.

[54] S. V. Patankar and D. Spalding. A calculation procedure for heat, mass and momentum transfer in three dimensional parabolic flows. Int. J. Heat Mass Transfer, 15:1787-1806, 1972.

[55] T. J. Poinsot and S. K. Lele. Boundary conditions for direct simulations of compressible viscous flows. J. Comput. Phys., 101:104-129, 1992. 
[56] R. Radebaugh, J. Zimmerman, D. R. Smith, and B. Louie. Comparison of three types of pulse tube refrigerators: New methods for reaching 60K. Advances in Cryogenic Engineering, 31:779-789, 1986.

[57] B. R. Ramaprian and S.W. Tu. An experimental study of oscillatory pipe flow at transitional Reynolds numbers. J. Fluid Mech., 100:513-544, 1980.

[58] E. G. Richardson and E. Tyler. The transverse velocity gradient near the mouths of pipes in which an alternating or continuous flow of air is established. Proc. Phys. Soc. London, 42:1-15, 1929.

[59] Y. Saad. Iterative Methods for Sparse Linear Systems. PWS Publishing, London, 1996.

[60] W. E. Schiesser and C. A. Silebi. Computational Transport Phenomena. Cambridge University Press, 1997.

[61] S. I. Sergeev. Fluid oscillations in pipes at moderate Reynolds numbers. Fluid Dyn., 1:121-122, 1966.

[62] T. Sexl. Über den von E.G. Richardson entdeckten "Annulareffekt". Zeitschrift für Physik, 61:349-362, 1930.

[63] L. Shemer, I. Wygnanski, and E. Kit. Pulsating pipe flow. J. Fluid Mech., 153:313337, 1985.

[64] M. Shiraishi, T. Ikeguchi, M. Murakami, A. Nakano, and T. Iida. Visualization of oscillatory flow phenomena in tapered pulse tube refrigerators. Advances in Cryogenic Engineering, 47:768-775, 2002.

[65] W. R. Smith. One-dimensional models for heat and mass transfer in pulse tube refrigerators. Cryogenics, 41:573-582, 2001.

[66] P. P. Steijaert. Thermodynamical aspects of pulse-tube refrigerator. PhD thesis, Eindhoven University of Technology, 1999.

[67] G. W. Swift. Thermoacoustic engines. J. Acoust. Soc. Am, 84:1145-1180, 1988.

[68] P. Szymanski. Some exact solutions of the hydrodynamic equations for viscous fluid in the case of a cylindrical tube. Journal de Mathematiques Pures et Appliquees, 11(9):67-107, 1932.

[69] I. A. Tanaeva. Low-temperature cryocooling. PhD thesis, Eindhoven University of Technology, 2004.

[70] S. F. Tardu and G. Binder. Wall shear stress modulation in unsteady turbulent channel flow. Physics of Fluids A, 5:2028-2037,1993.

[71] G. Thiart. Improved finite difference scheme for the solution of convectiondiffusion problems with the SIMPLEN algorithm. Numerical Heat Transfer, Part $B, 18: 81-95,1990$. 
[72] R. A. Trompert and J. G. Verwer. Analysis of the implicit Euler local uniform grid refinement method. SIAM J. Sci. Comput., 18:259-278, 1993.

[73] A. T. A. M. de Waele. Optimization of pulse tubes. Cryogenics, 39(1):13-15, 1999.

[74] A. T. A. M. de Waele. Pulse tube performance at very low temperatures. Physica B, Condensed Matter, 284-288:2018-2019, 2000.

[75] A. T. A. M. de Waele. Pulse-tube refrigerators: principle, recent developments and prospects. Physica B, Condensed Matter, 280:479-482, 2000.

[76] A. T. A. M. de Waele. Regenerator dynamics in the harmonic approximation. Cryogenics, 41(3):195-200, 2001.

[77] A. T. A. M. de Waele, P. P. Steijaert, and J. Gijzen. Thermodynamical aspects of pulse tubes I. Cryogenics, 37(6):313-324, 1997.

[78] A. T. A. M. de Waele, P. P. Steijaert, and J. Gijzen. Thermodynamical aspects of pulse tubes II. Cryogenics, 38(3):329-335, 1998.

[79] A. T. A. M. de Waele, I. A. Tanaeva, and Y. L. Yu. Multistage pulse tubes. Cryogenics, 40(7):459-464, 2000.

[80] A. T. A. M. de Waele, M. Y. Xu, and Y. L. Ju. Nonideal-gas effect in regenerators. Cryogenics, 39(10):847-851, 1999.

[81] C. Wang. Numerical analysis of $4 \mathrm{~K}$ pulse tube coolers: Part 1. numerical simulation. Cryogenics, 37:207-213, 1997.

[82] C. Wang. Numerical analysis of $4 \mathrm{~K}$ pulse tube coolers: Part 2. performances and internal processes. Cryogenics, 37:215-220, 1997.

[83] C. Wang, G. Thummes, and C. Heiden. Control of DC gas flow in a single-stage double-inlet pulse tube cooler. Cryogenics, 38:843-847, 1998.

[84] C. Wang, P. Wu, and Z. Chen. Numerical modelling of an orifice pulse tube refrigerator. Cryogenics, 32:785-790, 1992.

[85] P. Wesseling. Principles of Computational Fluid Dynamics. Springer, 2001.

[86] F. M. White. Viscous Fluid Flow. McGraw-Hill, 1974.

[87] M. E. Will, I. A. Tanaeva, R. Li, and A. T. A. M. de Waele. New rotary valves for pulse-tube refrigerators. Cryogenics, 44(11):793-800, 2004.

[88] D. W. J. Willems and J. A. M. Dam. Three-dimensional pulse tube simulations. Advances in Cryogenic Engineering, 47:934-941, 2001.

[89] K. Witzig. Über erzwungene Wellenbewegungen zäher, inkompressibler Flüssigkeiten in elastischen Röhren. Inaugural Dissertation der hohen philosophischen Fakultät, Bern, Switzerland, 1914. 
[90] M. Y. Xu, A. T. A. M. de Waele, and Y. L. Ju. A pulse tube refrigerator below $2 \mathrm{~K}$. Cryogenics, 39(6):865-869, 1999.

[91] H. Yaling, G. Chengming, X. Mingyao, C. Zhongqi, and T. Wenquan. Numerical simulation of convergent and divergent tapered pulse tube cryocoolers and experimental verification. Cryogenics, 41:699-704, 2001.

[92] T. S. Zhao and P. Cheng. Experimental investigations of the onset of turbulence and frictional losses in an oscillatory pipe flow. Int. J. of Heat and Fluid Flow, 17:356-362, 1996.

[93] T. S. Zhao and P. Cheng. The friction coefficient of a fully developed laminar reciprocating flow in a circular pipe. Int. J. of Heat and Fluid Flow, 17:167-172, 1996.

[94] T. S. Zhao and P. Cheng. Oscillatory heat transfer in a pipe subjected to a laminar reciprocating flow. ASME Journal of Heat Transfer, 118:592-598, 1996.

[95] S. Zhu, P. Wu, and Z. Chen. Double inlet pulse tube refrigerator: an important improvement. Cryogenics, 330:514-520, 1990.

[96] W. Zielke. Frequency dependent friction in transient pipe flow. Trans. ASME, J. of Basic Eng., 90:109-115, 1968. 


\section{Nomenclature}

\section{Roman symbols}

\begin{tabular}{|c|c|c|}
\hline$A_{t}$ & cross-sectional area of tube & {$\left[\mathrm{m}^{2}\right]$} \\
\hline$A_{w}$ & wall area & {$\left[\mathrm{m}^{2}\right]$} \\
\hline $\mathrm{C}_{\mathrm{or}}$ & flow conductance of the orifice & {$\left[\mathrm{m}^{3} / \mathrm{Pa} \cdot \mathrm{s}\right]$} \\
\hline$c_{f}$ & friction factor & {$\left[\mathrm{Pa} \cdot \mathrm{s} / \mathrm{m}^{2}\right]$} \\
\hline$c_{p}$ & constant pressure gas heat capacity & {$[\mathrm{J} / \mathrm{kg} \cdot \mathrm{K}]$} \\
\hline$c_{v}$ & constant volume gas heat capacity & {$[\mathrm{J} / \mathrm{kg} \cdot \mathrm{K}]$} \\
\hline$c_{w}$ & wall heat capacity & {$[\mathrm{J} / \mathrm{kg} \cdot \mathrm{K}]$} \\
\hline D & tube inner diameter & {$[\mathrm{m}]$} \\
\hline$\dot{\mathrm{E}}$ & energy flow & {$[\mathrm{W}]$} \\
\hline$\dot{\mathrm{H}}$ & enthalpy flow & {$[\mathrm{W}]$} \\
\hline $\mathrm{h}$ & convective heat transfer coefficient & {$\left[\mathrm{W} / \mathrm{m}^{2} \cdot \mathrm{K}\right.$} \\
\hline $\mathrm{f}$ & frequency & {$[\mathrm{Hz}]$} \\
\hline$k_{g}$ & gas thermal conductivity & {$[\mathrm{W} / \mathrm{m} \cdot \mathrm{K}]$} \\
\hline$k_{w}$ & wall thermal conductivity & {$[\mathrm{W} / \mathrm{m} \cdot \mathrm{K}]$} \\
\hline L & tube length & {$[\mathrm{m}]$} \\
\hline$l$ & wall thickness & [m] \\
\hline$\dot{m}$ & mass flow & {$[\mathrm{kg} / \mathrm{s}]$} \\
\hline $\mathrm{P}$ & driving pressure & {$[-]$} \\
\hline$p_{a v}$ & average pressure & {$[\mathrm{Pa}]$} \\
\hline $\bar{p}$ & pressure oscillation amplitude & {$[\mathrm{Pa}]$} \\
\hline$p_{b}$ & buffer pressure & {$[\mathrm{Pa}]$} \\
\hline$p_{t}$ & tube pressure & {$[\mathrm{Pa}]$} \\
\hline$\overline{\mathrm{Q}}_{i n t}$ & total interface heat flow & {$[\mathrm{W}]$} \\
\hline$\overline{\mathrm{Q}}_{g \text { as }}$ & total gas heat flow & {$[\mathrm{W}]$} \\
\hline$\overline{\mathrm{Q}}_{\text {wall }}$ & total wall heat flow & {$[\mathrm{W}]$} \\
\hline $\mathrm{Q}_{\mathrm{H}}$ & heat extracted at the hot end & {$[\mathrm{W}]$} \\
\hline$\dot{Q}_{c}$ & heat loaded at the cold end & [W] \\
\hline$q_{\text {int }}$ & heat flux at the interface & {$\left[\mathrm{W} / \mathrm{m}^{2}\right]$} \\
\hline$q_{\text {gas }}$ & heat flux in the gas & {$\left[\mathrm{W} / \mathrm{m}^{2}\right]$} \\
\hline$q_{\text {wall }}$ & heat flux in the wall & {$\left[\mathrm{W} / \mathrm{m}^{2}\right]$} \\
\hline $\mathrm{R}_{\mathrm{m}}$ & specific gas constant & {$[\mathrm{J} / \mathrm{kg} \cdot \mathrm{K}]$} \\
\hline
\end{tabular}


$R_{0}$

$\mathrm{R}_{1}$

$r$

$T$

$\mathrm{T}_{w}$

$\mathrm{T}_{\mathrm{a}}$

$\mathrm{T}_{\mathrm{C}}$

$\mathrm{T}_{\mathrm{H}}$

$t_{c}$

$u$

$v$

$\mathrm{V}_{\mathrm{b}}$

$\dot{V}_{\mathrm{H}}$

$x$

$z$

\section{Greek symbols}

$\alpha$
$\beta$
$\gamma$
$\delta_{k}$
$\delta$
$\epsilon$
$\rho$
$\mu$
$v$
$\tau$
$\omega$
$\Phi$
$\Psi$

\section{Dimensionless}

Fo

$\mathrm{Ma}$

M

$\mathrm{Nu}$

$\mathrm{Pe}$

Pr

Re

$\operatorname{Re}_{\omega}$

$\operatorname{Re}_{\delta}$

Va

$\lambda$

$\mathcal{A}$ tube inner radius

[m]

tube outer radius

[m]

radial coordinate

[m]

gas temperature

$[\mathrm{K}]$

$[\mathrm{K}]$

$[\mathrm{K}]$

ambient temperature

$[\mathrm{K}]$

[m]

[m]

$[\mathrm{m}]$

[m]

$[\mathrm{m}]$

$\left[\mathrm{m}^{3} / \mathrm{s}\right]$

[m]

[m]

axial coordinate in $2 \mathrm{D}$

thermal diffusivity coefficient

$[-]$

ratio of thermal conductivities

$[-]$

heat capacity ratio

thermal penetration depth

$[-]$

$[\mathrm{m}]$

Stokes layer thickness

[m]

diffusion coefficient

gas density

dynamic viscosity

$[-]$

$\left[\mathrm{kg} / \mathrm{m}^{3}\right]$

$[\mathrm{Pa} \cdot \mathrm{s}]$

kinematic viscosity

$\left[\mathrm{m}^{2} / \mathrm{s}\right]$

stress tensor

$[-]$

angular frequency

$[1 / \mathrm{s}]$

viscous dissipation function

$[-]$

flux limiter

$[-]$

Fourier number

Mach number

modified Mach number

Nusselt number

Peclet number

Prandtl number

Reynolds number

kinetic Reynolds number

Reynolds number based on $\delta$

Valensi number

Womersley number

pressure ratio 
$\mathcal{B}$

C

$\mathcal{D}$

$\mathcal{E}$

$\mathrm{J}_{0}$

$\mathrm{J}_{1}$

$\mathcal{P}$

$\Theta$

$x_{\mathrm{r}}$

Abbreviations

AC

BC

BICGSTAB

CFD

CFL

CHX

DC

HHX

FEM

FDM

FVM

G-M

LU

LUGR

MRI

PDE

PISO

PTR

rhs

SIMPLE

SIMPLEC

SIMPLEN

SIMPLER

TVD

1D,2D,3D

\section{Superscripts}

-- short bar

-- long bar

- hat

- dot constant

constant

constant

constant

Bessel function

Bessel function

stretching parameter

stretching parameter

reattachment length

aftercooler

boundary conditions

bi-conjugate gradients stabilised: iterative method

computational fluid dynamics

Courant-Friedrichs-Lewy number

cold heat exchanger

direct current: net flow

hot heat exchanger

finite element method

finite differences method

finite volume method

Gifford-McMahon

lower-upper: matrix decomposition

local uniform grid refinement

magneto-resonance imaging

partial differential equation

pressure implicit with splitting of operators

pulse tube refrigerator

right-hand side

semi-implicit method for pressure-linked equations

SIMPLE-consistent

SIMPLE-nonstaggered

SIMPLE-revised

total variation diminishing

dimensions

typical values for input data

time-averaged

dimensionless

time derivative 


\section{Subscripts}

$\begin{array}{ll}\text { a } & \text { ambient } \\ \text { av } & \text { cross-sectional averaged } \\ \text { b } & \text { buffer } \\ \text { C } & \text { cold } \\ \text { g } & \text { gas } \\ \text { H } & \text { hot } \\ \text { int } & \text { interface } \\ \text { w } & \text { wall } \\ 0 & \text { leading order } \\ 1 & \text { first order }\end{array}$




\section{Index}

aftercooler, 9

anelastic approximation, 3

asymptotic analysis, 18

backward-facing step flow, 74

basic pulse tube, 1

Bessel functions, 70, 71

BICGSTAB, 46, 84

boundary conditions

numerical, 38

physical, 31

buffer, 10

CFL stability condition, 36, 61

cold heat exchanger, 9

collocated grid, 40

conservation of energy, 13

conservation of mass, 13

conservation of momentum, 13

constraint, 27

convective heat transfer, 23

cooling power, 12

Courant number, 36, 60

density, 13

displacement length, 17

divergence operator, 13

double-inlet pulse tube, 1

dynamic viscosity, 14

enthalpy flow, 12, 88

equation of state, 13

external force, 13

finite difference method, 34 finite element method, 34

finite volume method, 34

first-order system, 18

flux limiter, 36

minmod, 36, 63

modified van Leer, 62

van Leer, 36, 62

Fourier number, 29

friction factor, 23

gradient operator, 13

Graetz problems, 77

Hagen-Poiseuille flow, 67

harmonic analysis, 3

heat flux, 13, 77, 96

high-resolution scheme

$1 \mathrm{D}, 35$

2D, 60

hot heat exchanger, 9

Lagrange multiplier, 30

leading-order system, 18, 26

linear resistance law, 21

low-Mach-number flow, 18

LU factorisation, 46, 84

LUGR, 52

fixed refinement area, 53

moving refinement area, 55

Mach number, 15

mass flow, 88

material derivative, 13

NumLab, 103 
orifice, 10

orifice pulse tube, 1

Peclet number, 15

Poisson law, 21

Prandtl number, 15

preconditioner, 46,84

pressure, 13

checkerboard, 43

hydrodynamic, 27

thermodynamic, 19

pressure correction, 37

PISO, 38

Poisson equation, 49

SIMPLE, 37

pulsating flow, 12

quasi-uniform grid, 58

reattachment length, 76

reciprocating flow, 12

regenerator, 9

Reynolds number, 15

based on Stokes layer, 16

kinetic, 15, 71

Riccati equation, 22

Richardson's annular effect, 74

specific heat, 13

stability condition, 60

staggered grid, 40

Stokes layer thickness, 16

Stokes parameter, 16

stress tensor, 13

stretching function, 58

tapered tube, 3

temperature, 13

thermal conductivity, 14

thermal diffusivity, 29

turbulence, 16

TVD, 61

Valensi number, 16

velocity, 13

viscous dissipation, 13 viscous stress tensor, 14, 24

volume flow, 21

wall model, 29

weakly compressible flow, 18

Womersley number, 16 


\section{Summary}

A new numerical model has been developed for simulating oscillating gas flow and heat transfer in the tube section of a pulse-tube refrigerator. Pulse-tube refrigerators are among the newest types of cryocoolers. They work by the cyclic compression and expansion of gas, usually helium. Introduced in 1963, pulse-tube refrigerators typically reached temperatures of about $120 \mathrm{~K}$. By the end of the 1990s temperatures below $2 \mathrm{~K}$ had been reached. The practical use of pulse-tube cryocoolers is still at an early stage. However, they are beginning to replace the older types of cryocoolers in a wide variety of applications: military, aerospace and medical industries. Advantages such as simplicity, low cost and reliability, combined with high performance, have resulted in an extensive study of pulse tubes in recent years.

The first and second laws of thermodynamics have been major tools to investigate pulse-tube refrigerators theoretically. However, a clearer understanding of the fluid dynamical properties is necessary if one wishes to make quantitative improvements in pulse tube performance. In this study we concentrate solely on the tube section of the pulse-tube refrigerator to identify undesired effects that occur in the tube and reduce the efficiency of the coolers.

The developed mathematical model is based on the conservation of mass, momentum and energy, and the equation of state. The conservation equations for compressible viscous unsteady flow are written in differential form using primitive variables. One-dimensional and two-dimensional cylindrical axisymmetrical cases are considered. According to dimensional analysis, the tube conveys a low-Mach-number compressible flow. Therefore, we expanded all relevant variables in terms of powers of $\mathrm{M}^{2}$, a parameter related to the Mach number. This asymptotic consideration reveals several key features of pulse tube flow. Two physically distinct roles of pressure are to be distinguished: one as thermodynamic variable and one as hydrodynamic variable. The thermodynamical pressure appears in the energy equation and in the equation of state. It is spatially uniform, thus a function of time only, and is responsible for the global compression and expansion. The hydrodynamical pressure appears in the momentum equations and is induced by inertia and viscous forces. The acoustic pressure does not play a role in pulse tubes. Due to the non-linearity of the resulting system of equations, general analytical solutions are not available. Therefore numerical modelling has been applied.

For the numerical solution of the resulting system of equations finite difference 
methods are used. The energy equation for the temperature is a convection-diffusion equation, mostly of a convective nature. It is solved with state-of-the-art flux-limiter schemes in an attempt to preserve the steep temperature gradients in a pulse tube. When large gradients are present, either internally or adjacent to a boundary, more accurate solutions can be obtained by grid refinement. Refining a grid throughout the entire computational domain can be expensive, particularly in multi-dimensions. Instead of applying non-uniform locally refined grids, we use several uniform grids with different mesh sizes that cover different parts of the domain. One coarse grid covers the entire domain. The mesh size of this global grid is chosen according to the smoothness of the solution outside the high-activity regions. Besides the global grid, fine local grids are used which are also uniform. They cover only parts of the domain and contain the high-activity regions. The mesh size of each of these grids follows the activity of the solution. The solution is approximated on the composite grid which is the union of the uniform subgrids. This refinement strategy is known as local uniform grid refinement (LUGR).

To deal with the problem of pressure-velocity coupling in the flow computation, we employ a pressure correction method. It is specially designed for low-Mach-number compressible flows. Combining the continuity equation and the energy equation, we derive an expansion equation or velocity divergence constraint. Our pressure correction scheme is based on this expansion equation and not on the continuity equation, which is different from the common approach in the simulation of compressible flows.

The simulation tool, based on the proposed model, is constructed and tested on classic problems with known analytical solutions. Finally, the model was applied to a typical pulse-tube refrigerator. Results of one-dimensional and two-dimensional axisymmetrical simulations are presented and interpreted. The proposed model is more accurate and versatile than the widely used harmonic analysis and computationally less expensive than a full three-dimensional simulation with commercially available codes. It can be used for practical simulations, for calculating optimal values of the real system design parameters and for investigating different physical effects in the pulse tube. 


\section{Samenvatting}

Een nieuw numeriek model is ontwikkeld voor de simulatie van oscillerende gas stromen en warmtetransport in het buisgedeelte van een pulsbuiskoelmachine. Pulsbuiskoelmachines behoren tot de nieuwste types lage-temperatuur-koelers. Ze werken door middel van de cyclische compressie en expansie van gas, meestal helium. De pulsbuiskoeler zoals geïntroduceerd in 1963 bereikte temperaturen van $120 \mathrm{~K}$. Tegen het eind van de jaren 1990 werden al temperaturen beneden $2 \mathrm{~K}$ bereikt. Het gebruik van pulsbuiskoelers in de praktijk bevindt zich nog steeds in een beginstadium. Echter, ze beginnen koelers van een ouder type te vervangen in een breed scala aan toepassingen in de militaire, ruimtevaart- en medische industrie. Voordelen als eenvoud, lage kosten en betrouwbaarheid, gecombineerd met hoge prestaties, hebben in de afgelopen jaren geresulteerd in uitgebreid onderzoek aan pulsbuiskoelers.

De eerste twee hoofdwetten van de thermodynamica zijn altijd het belangrijkste gereedschap geweest in theoretische studies van pulsbuiskoelers. Echter, een beter begrip van de stromingstechnische eigenschappen is nodig indien men de prestaties van de pulsbuiskoeler kwantitatief wenst te verbeteren. In dit onderzoek concentreren we ons puur op het buisgedeelte van een pulsbuiskoelmachine om ongewenste effecten die in de buis optreden, en die de efficiency van de koeler reduceren, te identificeren.

Het ontwikkelde wiskundige model is gebaseerd op het behoud van massa, impuls en energie, en op de toestandsvergelijking van het gas. De behoudsvergelijkingen voor samendrukbare viskeuze instationaire stroming zijn met primitieve variabelen geschreven in differentiaalvorm. Eendimensionale en tweedimensionale cilindrische axiaalsymmetrische situaties zijn beschouwd. Dimensieanalyse geeft aan dat de buis een samendrukbare doorstroming met klein getal van Mach heeft. Daarom hebben wij alle relevante variabelen in termen van machten van $\mathrm{M}^{2}$ geschreven, waarbij $\mathrm{M}$ een gemodificeerd Mach getal is. Deze asymptotische beschouwing legt verschillende belangrijke eigenschappen van pulsbuisstroming bloot. Twee fysisch verschillende functies van druk kunnen worden onderscheiden: de ene als thermodynamische variabele en de ander als hydrodynamische variabele. De thermodynamische druk verschijnt in de energievergelijking en in de toestandsvergelijking. Deze is ruimtelijk uniform, dus alleen een functie van de tijd, en verantwoordelijk voor de globale compressie en expansie. De hydrodynamische druk verschijnt in de impulsvergelijking en wordt veroorzaakt door traagheids- en viskeuze krachten. De 
akoestische druk speelt geen rol in pulsbuizen. Vanwege de niet-lineariteit van het resulterende stelsel vergelijkingen zijn algemene analytische oplossingen niet beschikbaar. Daarom is numerieke modellering toegepast.

Voor de numerieke oplossing van het resulterende stelsel van vergelijkingen zijn eindige-differentie-methoden gebruikt. De energievergelijking voor de temperatuur is een convectie-diffusie vergelijking, voornamelijk van convectieve aard. Deze is opgelost met moderne "flux-limiter" schema's in een poging de grote temperatuurgradiënten in de pulsbuis numeriek in stand te houden. Wanneer grote gradiënten optreden, in het binnengebied of bij een rand, dan kunnen met behulp van roosterverfijning nauwkeurigere oplossingen worden verkregen. Het verfijnen van een rooster op het gehele rekenkundige domein kan duur zijn, in het bijzonder op een twee- of driedimensionaal domein. In plaats van het toepassen van niet-uniforme lokaal verfijnde roosters gebruiken wij een aantal uniforme roosters met verschillende maaswijdtes die verschillende delen van het domein bedekken. Een grof rooster bedekt het hele domein. De maaswijdte van dit globaal rooster is gekozen in overeenstemming met de gladheid van de oplossing buiten de gebieden met hoge activiteit. Naast het globale rooster zijn fijne lokale roosters gebruikt die ook uniform zijn. Zij bedekken alleen die gedeeltes van het domein met hoge activiteit. De maaswijdte van elk van deze roosters volgt de activiteit van de lokale oplossing. De oplossing wordt benaderd op het samengestelde rooster dat een vereniging is van de uniforme deelroosters. Deze verfijningsstrategie staat bekend als "local uniform grid refinement" (LUGR).

Om het probleem van druk-snelheid koppeling in de stroming aan te pakken, gebruiken we een druk-correctie-methode. Deze is speciaal ontworpen voor samendrukbare stromingen met klein Mach-getal. We combineren de continuïteitsvergelijking met de energievergelijking en leiden daaruit een expansievergelijking (of drukdivergentie voorwaarde) af. Ons druk-correctie schema is gebaseerd op deze expansievergelijking en niet op de continuïteitsvergelijking, hetgeen verschilt met de conventionele aanpak in de simulatie van samendrukbare stromingen.

Een computerprogramma, gebaseerd op het door ons opgezette model, is geïmplementeerd en getest op klassieke problemen met bekende analytische oplossingen. Tenslotte is het simulatiemodel toegepast op de pulsbuiskoeler. Resultaten van eendimensionale en tweedimensionale axiaalsymmetrische simulaties worden getoond en geïnterpreteerd. Het ontwikkelde model is nauwkeuriger en veelzijdiger dan de veelgebruikte harmonische analyse en rekenkundig minder duur dan een complete driedimensionale simulatie met commercieel verkrijgbare software. Het computerprogramma kan worden gebruikt voor praktische simulaties, voor het berekenen van optimale waarden voor de werkelijke ontwerpparameters en voor het onderzoeken van verscheidene fysische verschijnselen in de pulsbuis. 


\section{Acknowledgements}

This research has been a joint project between the Department of Applied Physics, Stirling Cryogenics and Refrigeration B.V. and the Department of Mathematics and Computer Science. I would like to thank all people who participated in this project. I am especially grateful to my supervisors Prof. Dr. R.M.M. Mattheij, Head of the Scientific Computing Group, and Prof. Dr. A.T.A.M. de Waele, Head of the Low Temperature Physics Group, for their guidance, stimulating support and encouragement. I would like to express my warmest thanks to my copromotor Dr. Ir. Arris Tijsseling to whom I could come any time with all my questions, problems and achievements.

This research project was partly sponsored by Stirling Refrigeration and Cryogenics B.V. and I would like to thank Ir. Ronald den Heijer, MBM (director) and Dr. Jacques Dam (former engineering manager) for their interest in my work. A special word of thank to Daniel Willems for our regular meetings and fruitful discussions which helped me in understanding the physics behind all these equations, formulae and numbers.

The contribution of Dr. Warren Smith (University of Birmingham, UK) in the beginning of the project was very valuable. This allowed me to make a quick start and to get first numerical results in quite a short time. I also want to thank Dr. Ir. Jan ten Thije Boonkkamp for his practical advices and useful suggestions concerning flux limiters and pressure correction algorithms. The contents of the thesis have been improved due to the comments and constructive remarks of Prof. Dr. A.E. Vardy (University of Dundee, UK) and Prof. Dr. Y. Matsubara (Nihon University, Japan).

I have highly appreciated the excellent research facilities and the very friendly work atmosphere in our group. I would like to thank all present and former members of the Scientific Computing Croup, especially my room mates Bratislav Tasic, Wienand Drenth and William Dijkstra. I have benefited a lot from the frequent contacts with my colleagues, among them I would like to mention Bas van der Linden, Paul de Haas, Martijn Anthonissen, Marialuce Graziadei, Christina Giannopapa, Konstantin Laevsky, Seva Nefedov, Mischa Sizov and Pavel Kagan. They were always ready to help. Besides, I shall keep nice memories of our informal discussions during the lunches, coffee-breaks and outings.

Finally, I am very grateful to my husband Alexey and to my daughter Katya for their loving support and understanding. 


\section{Curriculum Vitae}

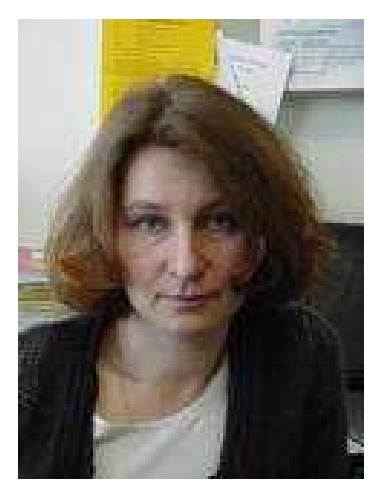

Irina Lyulina was born in St. Petersburg, Russia on March 5, 1968. She finished her high-school education in 1985 and in the same year she started her studies at the Department of Mathematics and Mechanics, St. Petersburg State University. In 1990 she obtained the title "Master of Science" in Applied Mathematics. In 1993 she completed her post-graduate studies at the same University, specialising in numerical analysis and computational mathematics. From 1994 she has been moving across Europe with her family, living in Belgium, Germany, United Kindom and, finally, The Netherlands. During her stay in the UK, from 1998 to 2000, she was a visitor at the Department of Applied Mathematics, University of Leeds, and did a one year post-graduate course at the same University in the School of Computing. In 2000 she became a PhD student in the Scientific Computing Group, Department of Mathematics and Computer Science, Eindhoven University of Technology. 